DESY 11-002

April 2011

\title{
Entropy, Baryon Asymmetry and Dark Matter from Heavy Neutrino Decays
}

\author{
W. Buchmüller, K. Schmitz, G. Vertongen \\ Deutsches Elektronen-Synchrotron DESY, 22607 Hamburg, Germany
}

\begin{abstract}
The origin of the hot phase of the early universe remains so far an unsolved puzzle. A viable option is entropy production through the decays of heavy Majorana neutrinos whose lifetimes determine the initial temperature. We show that baryogenesis and the production of dark matter are natural by-products of this mechanism. As is well known, the cosmological baryon asymmetry can be accounted for by leptogenesis for characteristic neutrino mass parameters. We find that thermal gravitino production then automatically yields the observed amount of dark matter, for the gravitino as the lightest superparticle and typical gluino masses. As an example, we consider the production of heavy Majorana neutrinos in the course of tachyonic preheating associated with spontaneous $B-L$ breaking. A quantitative analysis leads to constraints on the superparticle masses in terms of neutrino masses: For a light neutrino mass of $10^{-5} \mathrm{eV}$ the gravitino mass can be as small as $200 \mathrm{MeV}$, whereas a lower neutrino mass bound of $0.01 \mathrm{eV}$ implies a lower bound of $9 \mathrm{GeV}$ on the gravitino mass. The measurement of a light neutrino mass of $0.1 \mathrm{eV}$ would rule out heavy neutrino decays as the origin of entropy, visible and dark matter.
\end{abstract}




\section{Contents}

1 Introduction 3

2 Flavour model and leptogenesis

3 Entropy production through neutrino decays $\quad \mathbf{1 0}$

3.1 Initial conditions . . . . . . . . . . . . . . . . . . . . 10

3.2 Boltzmann equations . . . . . . . . . . . . . . . . . 13

3.2.1 Collision operators . . . . . . . . . . . . . . . 13

3.2 .2 Higgs bosons $S \ldots \ldots \ldots \ldots$

3.2.3 Heavy Majorana neutrinos $N_{1} \ldots \ldots \ldots \ldots$

$3.2 .4 \quad B-L$ asymmetry . . . . . . . . . . . . . . . . . . . . . . . . . 19

3.2.5 Radiation $R$. . . . . . . . . . . . . . . . . . . . . 20

3.2 .6 Gravitinos $\widetilde{G} \ldots \ldots \ldots \ldots 21$

4 An illustrative example $\quad 22$

4.1 Comoving number and energy densities . . . . . . . . . . . . . . 24

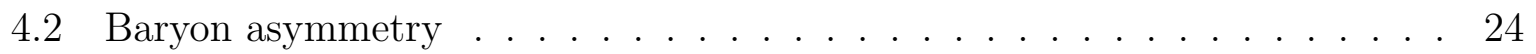

4.3 Radiation temperature . . . . . . . . . . . . . . . . . . . 27

4.4 Gravitino dark matter . . . . . . . . . . . . . . . . . 29]

5 Results and discussion 31

5.1 Reheating temperature . . . . . . . . . . . . . . . 32

5.2 Baryon asymmetry . . . . . . . . . . . . . . . . 35

5.3 Gravitino dark matter . . . . . . . . . . . . . . . . . 4 40

6 Summary and outlook 47

A Conventions for the Boltzmann equations $\quad 49$

B Phase space distribution of thermal neutrinos

C Reheating temperature $\quad 53$

D Semi-analytic reconstructions 


\section{Introduction}

Detailed studies of the cosmic microwave background provide direct evidence for the hot thermal universe close to its minimal temperature [1]. The extrapolation to higher temperatures, beyond primordial nucleosynthesis, is very uncertain, and we do not know how large the maximal temperature of the hot early universe has been. It is widely believed that the universe was 'reheated' by a transition from a preceding inflationary phase where 'vacuum energy' dominated the expansion [2]. Knowing the resulting reheating temperature is of fundamental importance since it is closely related to the origin of the matter-antimatter asymmetry and the nature of dark matter.

In a recent paper we have suggested that the entropy of the hot early universe was produced in the decays of heavy Majorana neutrinos whose lifetimes determine the reheating temperature [3]. We have demonstrated that the baryon asymmetry and dark matter then naturally result as by-products: The mechanism of baryogenesis is a mixture of thermal and nonthermal leptogenesis, and the dominant component of dark matter is the gravitino which is assumed to be the lightest superparticle.

Our work is closely related to previous studies of thermal leptogenesis [4/5] and nonthermal leptogenesis via inflaton decay [6,7, where the inflaton lifetime determines the reheating temperature. In supersymmetric models with global $B-L$ symmetry the scalar superpartner $\widetilde{N}_{1}$ of the lightest heavy neutrino $N_{1}$ can play the role of the inflaton in chaotic [8] or hybrid [9] inflationary models. Coherent $\widetilde{N}_{1}$ oscillations after inflation may even dominate the energy density of the universe [10]. Nonthermal leptogenesis can be realized at significantly smaller temperatures than thermal leptogenesis. In this way the 'gravitino problem' for heavy unstable gravitinos [11,12,13] can be easily avoided, which has been one of the main motivations of nonthermal leptogenesis.

It is well known that the high temperatures characteristic for thermal leptogenesis can become a virtue if the gravitino is the lightest superparticle (LSP). For superparticle masses as they arise in gravity or gaugino mediation, thermal production of gravitinos can then explain the observed amount of dark matter [14]. As pointed out in [3], the required high temperatures are indeed realized if the universe is reheated through the decays of the heavy Majorana neutrinos. The vacuum decay width of the lightest heavy Majorana neutrino is given by

$$
\Gamma_{N_{1}}^{0}=\frac{\widetilde{m}_{1}}{8 \pi}\left(\frac{M_{1}}{v_{\mathrm{EW}}}\right)^{2} \sim 10^{3} \mathrm{GeV},
$$

where we have used the typical values $M_{1} \sim 10^{10} \mathrm{GeV}$ for the $N_{1}$ neutrino mass, $\widetilde{m}_{1} \sim 0.01 \mathrm{eV}$ for the effective light neutrino mass and $v_{\mathrm{EW}}=174 \mathrm{GeV}$ for the vac- 
uum expectation value of electroweak symmetry breaking. The corresponding reheating temperature is given by

$$
T_{R H} \approx\left(\frac{90}{8 \pi^{3} g_{\star, \rho}}\right)^{1 / 4} \sqrt{\Gamma_{N_{1}}^{0} M_{P}} \sim 10^{10} \mathrm{GeV},
$$

where we have used $g_{\star, \rho} \sim 200$ for the effective number of relativistic degrees of freedom, and $M_{P}=1.22 \times 10^{19} \mathrm{GeV}$ is the Planck mass.

From Eqs. (1) and (2) one obtains the reheating temperature $T_{R H}$ in terms of the neutrino masses $\widetilde{m}_{1}$ and $M_{1}$, the two key parameters for thermal and nonthermal leptogenesis. Assuming the gluino to be the heaviest gaugino, thermal gravitino production is dominated by QCD processes, which yields the gravitino abundance in terms of the gravitino mass $m_{\widetilde{G}}$ and the gluino mass $m_{\tilde{g}}$,

$$
\Omega_{\widetilde{G}} h^{2}=C\left(\frac{T_{R H}}{10^{9} \mathrm{GeV}}\right)\left(\frac{10 \mathrm{GeV}}{m_{\widetilde{G}}}\right)\left(\frac{m_{\tilde{g}}}{1 \mathrm{TeV}}\right)^{2},
$$

where the coefficient $C=0.26$ to leading order in the gauge coupling [15,16, 1 . Since $T_{R H}$ depends on $\widetilde{m}_{1}$ and $M_{1}$, the requirement ${ }^{2} \Omega_{\widetilde{G}} h^{2}=\Omega_{\mathrm{DM}} h^{2} \simeq 0.11$ [20] yields a connection between neutrino and superparticle mass parameters. The neutrino masses $\widetilde{m}_{1}$ and $M_{1}$ are in turn constrained by the condition that the maximal baryon asymmetry is larger than the observed one, $\eta_{B} \geq \eta_{B}^{\text {obs }}=6.2 \times 10^{-10}[20]$.

In the following sections we shall study in detail the connection between neutrino and superparticle masses, which is implied by successful leptogenesis and gravitino dark matter. As an example, we shall consider tachyonic preheating [21, associated with $B-L$ breaking, as a mechanism which can lead to a phase where the energy density is dominated by heavy Majorana neutrinos. As we shall see, the final baryon asymmetry and the dark matter abundance can then be calculated in terms of several parameters of the Lagrangian, independent of initial conditions: the scale $v_{B-L}$ of $B-L$ breaking, the heavy Majorana neutrino mass $M_{1}$, the effective light neutrino mass $\widetilde{m}_{1}$, the gravitino mass $m_{\widetilde{G}}$ and the gluino mass $m_{\tilde{g}}$. Particularly interesting is the resulting connection between the lightest neutrino mass $m_{1}$ and the gravitino mass.

Our analysis requires a flavour model which is flexible enough to allow for a large range of the neutrino masses $M_{1}$ and $\widetilde{m}_{1}$, the crucial parameters for leptogenesis. Such a model is described in Section 2. Subsequently, in Section 3, we discuss in detail the Boltzmann

\footnotetext{
${ }^{1}$ Note that $C$ has an $\mathcal{O}(1)$ uncertainty due to unknown higher order contributions and nonperturbative effects [15]. Resummation of thermal masses increases $C$ by about a factor of two [17.

${ }^{2}$ For the superparticle masses considered in this paper the contribution to the gravitino abundance from the decay of the next-to-lightest superparticle (NLSP) is negligible [18|19].
} 
equations that describe the time evolution of the state produced in the tachyonic decay of the false vacuum with unbroken $B-L$ symmetry. A novel technical aspect of our analysis is the separate treatment of thermal and nonthermal contributions to the abundance of the heavy Majorana neutrinos.

Using the set of Boltzmann equations derived in Section 3, we study an illustrative example of our mechanism in Section 4. The parameters are chosen such that the nonthermal contribution to leptogenesis dominates. Particular emphasis is given to the time dependence of the various production and decay rates and the emergence of a plateau where the temperature is approximately constant. In Section 5 the analysis is extended to the entire parameter space. First, the ranges of $M_{1}$ and $\widetilde{m}_{1}$ are determined for which leptogenesis is successful. The observed dark matter abundance then constrains the superparticle masses in terms of the neutrino masses.

Our results are summarized in Section 6. The appendices deal with various technical aspects of our calculations: conventions for the Boltzmann equations in Appendix A, the distribution function of thermally produced neutrinos in Appendix B, analytical approximations for the reheating temperature in Appendix $\mathrm{C}$ and semi-analytical results for the gravitino abundance in Appendix D.

\section{$2 \quad$ Flavour model and leptogenesis}

In the following we shall describe a flavour model which describes masses and mixings of quarks, charged leptons and neutrinos, and which is flexible enough to allow for a large range of the neutrino parameters $M_{1}$ and $\widetilde{m}_{1}$ that are crucial for leptogenesis. The model is a variant of [22] and satisfies all constraints from flavour changing processes [23].

We consider the extension $\mathrm{SU}(3)_{c} \times \mathrm{SU}(2)_{L} \times \mathrm{U}(1)_{Y} \times \mathrm{U}(1)_{B-L}$ of the standard model gauge group. The Yukawa interactions of quarks and leptons with Higgs fields are described by the following superpotential for matter superfields,

$$
W_{M}=h_{i j}^{u} \mathbf{1 0}_{i} \mathbf{1 0}{ }_{j} H_{u}+h_{i j}^{d} \mathbf{5}_{i}^{*} \mathbf{1 0}_{j} H_{d}+h_{i j}^{\nu} \mathbf{5}_{i}^{*} n_{j}^{c} H_{u}+\frac{1}{2} h_{i}^{n} n_{i}^{c} n_{i}^{c} S .
$$

Here the standard model fermions have been arranged in $\mathrm{SU}(5)$ multiplets, $\mathbf{1 0}=\left(q, u^{c}, e^{c}\right)$ and $\mathbf{5}^{*}=\left(d^{c}, \ell\right)$, and $i, j=1 \ldots 3$ are flavour indices. $n^{c}$ contain $\nu_{R}^{c}$, the charge conjugates of the right-handed neutrinos, which are related to the heavy Majorana neutrinos $N$ through $N_{i}=\nu_{R i}+\nu_{R i}^{c}$. For simplicity, we have used SU(5) notation assuming that the colour triplet partners of the Higgs doublets have been projected out.

Vacuum expectation values of the scalar Higgs fields, $\left\langle H_{u, d}\right\rangle=v_{u, d}$, break the elec- 


\begin{tabular}{c|cccccccccccccc}
\hline \hline$\psi_{i}$ & $\mathbf{1 0}_{3}$ & $\mathbf{1 0}_{2}$ & $\mathbf{1 0}_{1}$ & $\mathbf{5}_{3}^{*}$ & $\mathbf{5}_{2}^{*}$ & $\mathbf{5}_{1}^{*}$ & $n_{3}^{c}$ & $n_{2}^{c}$ & $n_{1}^{c}$ & $H_{u}$ & $H_{d}$ & $S_{1}$ & $S_{2}$ & $T$ \\
\hline$Q_{i}$ & 0 & 1 & 2 & $a$ & $a$ & $a+1$ & $b$ & $c$ & $d$ & 0 & 0 & 0 & 0 & $e$ \\
\hline \hline
\end{tabular}

Table 1: Chiral U(1) charges.

troweak symmetry. The superpotential

$$
W_{B-L}=\frac{\sqrt{\lambda}}{2} T\left(v_{B-L}^{2}-2 S_{1} S_{2}\right)
$$

enforces $B-L$ breaking, with $\left\langle S_{1}\right\rangle=\left\langle S_{2}\right\rangle=v_{B-L} / \sqrt{2}$, via the supersymmetric Higgs mechanism. Using the field basis $S_{1}=S^{\prime} \exp (i \Phi) / \sqrt{2}, S_{2}=S^{\prime} \exp (-i \Phi) / \sqrt{2}$ and shifting around the vacuum expectation value, $S^{\prime}=v_{B-L}+S, S$ and $T$ have a common Dirac mass term, whereas $\Phi$ and the $B-L$ vector multiplet form together a massive vector multiplet. Since $B-L$ is gauged the inflaton is identified with the scalar component of the singlet field $T$. The potential for the scalar neutrinos is not sufficiently flat.

The pattern of Yukawa couplings $h_{i j}$ is determined by a Froggatt-Nielsen U(1) flavour symmetry, following Ref [22]. The matter fields $\psi_{i}$ and an extra singlet $\Sigma$ carry charges $Q_{i}$ and -1 under the flavour symmetry respectively, and are coupled together via effective non-renormalisable interactions associated with a scale $\Lambda>\Lambda_{\mathrm{GUT}}$. The Yukawa couplings are generated once the flavour symmetry is spontaneously broken by the expectation value of the $\Sigma$ field, and are given by

$$
h_{i j} \propto \eta^{Q_{i}+Q_{j}},
$$

where $\eta=\langle\Sigma\rangle / \Lambda$. The hierarchies of quark and lepton masses are then naturally obtained for $\eta^{2} \simeq 1 / 300$ using the chiral charges listed in Table 1. It is important to note that the Yukawa couplings are only specified up to factors of $\mathcal{O}(1)$. Cosmology further constrains the chiral charges. For instance, successful thermal leptogenesis requires $a+d=2[22$.

We shall restrict our analysis to the case of a hierarchical heavy neutrino mass spectrum, $M_{1} \ll M_{2,3}$, which is obtained for flavour charges of $N_{1}$ and $N_{2,3}$ separated by one unit, $b=c=d-1$. This is sufficient to illustrate our main point, the contraints imposed on the gravitino mass by neutrino masses. The lepton asymmetry will mostly be generated by decays of the lightest heavy neutrino $N_{1}$. The masses of the Majorana neutrinos are given by

$$
\begin{aligned}
& M_{1} \simeq \eta^{2 d} v_{B-L}, \\
& M_{2,3} \simeq \eta^{2(d-1)} v_{B-L},
\end{aligned}
$$


where $M_{2} / M_{3}=\mathcal{O}(1)$. The mass spectrum is now parametrised by the three remaining free charges $a, d$ and $e$, which can be related to the physical parameters $v_{B-L}, M_{1}$ and the Higgs mass $m_{S}$.

Using the Dirac mass matrix $m_{D}=h^{\nu} v_{u}$ and the Majorana mass matrix $M=h^{n} v_{B-L}$ derived from Eq. (4), the eigenvalues of the light neutrino mass matrix $m=-m_{D} M^{-1} m_{D}^{T}$ are given by

$$
\begin{aligned}
& m_{1} \simeq \eta^{2 a+2} \frac{v_{\mathrm{EW}}^{2}}{v_{B-L}} \\
& m_{2} \simeq m_{3} \simeq \eta^{2 a} \frac{v_{\mathrm{EW}}^{2}}{v_{B-L}}
\end{aligned}
$$

where we have used $\tan \beta=v_{u} / v_{d}>\mathcal{O}(1)$ and $v_{u} \simeq v_{\mathrm{EW}}$. Choosing $\bar{m}_{\nu}=\sqrt{m_{2} m_{3}} \simeq$ $\left|\left(m_{1}^{2}-m_{2}^{2}\right)\left(m_{2}^{2}-m_{3}^{2}\right)\right|^{1 / 4} \simeq 3 \times 10^{-2} \mathrm{eV}$ (cf. [1]), the $B-L$ breaking scale is determined by the flavour charge $a$,

$$
v_{B-L} \simeq \eta^{2 a} \frac{v_{\mathrm{EW}}^{2}}{\bar{m}_{\nu}},
$$

where $v_{\mathrm{EW}}^{2} / \bar{m}_{\nu} \simeq 10^{15} \mathrm{GeV}$ is the grand unification mass scale. Once $v_{B-L}$ is fixed, $M_{1}$ is directly related to the charge $d$ through Eq. (7a).

The ranges over which the chiral charges $a$ and $d$, and thus the physical parameters $v_{B-L}$ and $M_{1}$, are allowed to vary is restricted. First, the requirement that Yukawa couplings do not exceed the top-Yukawa coupling imposes the lower bounds $a \geq 0$ and $d \geq 1$. Furthermore, the upper bound $a \leq 1$ follows from $\tan \beta>\mathcal{O}(1)$. No corresponding upper bound on the charge $d$ exists but, as we shall see later, a CP asymmetry sufficiently large for successful leptogenesis requires $d<3$. Using Eq. (9), the allowed range of $B-L$ breaking scales reads

$$
3 \times 10^{12} \mathrm{GeV} \leq v_{B-L} \leq 1 \times 10^{15} \mathrm{GeV}
$$

For fixed $B-L$ breaking scale, the possible range of $M_{1}$ is given by Eq. (7a),

$$
\begin{array}{lll}
1 \times 10^{5} \mathrm{GeV} \leq M_{1} \leq 1 \times 10^{10} \mathrm{GeV} & \text { for } & v_{B-L}=3 \times 10^{12} \mathrm{GeV} \\
3 \times 10^{7} \mathrm{GeV} \leq M_{1} \leq 3 \times 10^{12} \mathrm{GeV} & \text { for } & v_{B-L}=1 \times 10^{15} \mathrm{GeV}
\end{array}
$$

The ranges for $v_{B-L}$ and $M_{1}$ correspond to a continuous variation of the flavour charges, which can effectively be realised by fractional charges. Note that the constraint from thermal leptogenesis, $a+d=2$, is now relaxed. 
Given the Yukawa couplings, one easily obtains the decay widths of the heavy Majorana neutrinos,

$$
\Gamma_{N_{i}}^{0} \simeq \frac{\widetilde{m}_{i}}{8 \pi} \frac{M_{i}^{2}}{v_{\mathrm{EW}}^{2}}
$$

with the effective light neutrino masses

$$
\widetilde{m}_{i}=\frac{1}{M_{i}}\left(m_{D}^{\dagger} m_{D}\right)_{i i} \simeq \eta^{2 a} \frac{v_{\mathrm{EW}}^{2}}{v_{B-L}} \simeq \bar{m}_{\nu} .
$$

The CP asymmetry in the heavy neutrino decays are given by [24]25]

$$
\epsilon_{i}=\frac{1}{8 \pi\left(h^{\nu \dagger} h^{\nu}\right)_{i i}} \sum_{j \neq i} \operatorname{Im}\left\{\left[\left(h^{\nu \dagger} h^{\nu}\right)_{i j}\right]^{2}\right\} F\left(\frac{M_{j}}{M_{i}}\right)
$$

where we use the standard model expression for $F 3^{3}$ Using our flavour model, one gets

$$
\epsilon_{1} \simeq 0.1 \eta^{2(a+d)}=0.1 \frac{\bar{m}_{\nu} M_{1}}{v_{\mathrm{EW}}^{2}}, \quad \epsilon_{2,3} \simeq \epsilon_{1} \eta^{-2} .
$$

Note that this is the maximal $\mathrm{CP}$ asymmetry for fixed $M_{1}$ [10]26], which is obtained in the limit $\widetilde{m}_{1} \rightarrow 0$. For $M_{1} \simeq 10^{10} \mathrm{GeV}$, this yields $\epsilon_{1} \sim 10^{-6}$. For other Majorana neutrino masses, the asymmetry scales like the mass ratio, $\epsilon_{1} \sim 10^{-6} M_{1} / 10^{10} \mathrm{GeV}$.

Since the light neutrino mass matrix is not hierarchical, the $\mathcal{O}(1)$ uncertainties in the $h^{\nu}$ Yukawa couplings can lead to large deviations from the relation (13) between $\widetilde{m}_{1}$ and $\bar{m}_{\nu}$. The only rigorous inequality is $\widetilde{m}_{1} \geq m_{1}$ [27]. We take these uncertainties into account by varying the effective neutrino mass in the range

$$
10^{-5} \mathrm{eV} \leq \widetilde{m}_{1} \leq 0.1 \mathrm{eV}
$$

Since the heavier Majorana neutrinos $N_{2,3}$ only play a marginal role in our scenario (see below), we ignore possible deviations from the relation (13) and use $\widetilde{m}_{2,3}=\bar{m}_{\nu}$.

In the following we consider the 'waterfall transition' from the false vacuum $\langle S\rangle=0$ to the true vacuum $\langle S\rangle=v_{B-L}$, which may happen at the end of hybrid inflation. A tachyonic instability in the Higgs potential leads to spinodal growth of the long-wavelength Higgs modes. The true vacuum is reached after a rapid transition at time $t_{\mathrm{PH}}$ [28],

$$
\left.\left\langle S^{\dagger} S\right\rangle\right|_{t=t_{\mathrm{PH}}}=v_{B-L}^{2}, \quad t_{\mathrm{PH}} \simeq \frac{1}{2 m_{S}} \ln \left(\frac{32 \pi^{2}}{\lambda}\right) .
$$

\footnotetext{
${ }^{3}$ The expression in the supersymmetric standard model would only slightly increase the value of $\epsilon_{i}$.
} 
The Higgs boson forms a massive supermultiplet together with a Dirac fermion and three additional bosons. Its mass and the false vacuum energy density are given by

$$
m_{S}^{2}=\lambda v_{B-L}^{2}, \quad \rho_{0}=\frac{1}{4} \lambda v_{B-L}^{4} .
$$

The size of the coupling $\lambda$ is determined by the flavour charge $e$ in Table 1 . We shall restrict our analysis to the case $e=2(d-1)$, such that $m_{S} \simeq M_{2,3}$. As a consequence, the Higgs boson only decays to pairs of $N_{1}$ neutrinos and not to pairs of $N_{2}$ or $N_{3}$ neutrinos. Like the heavy neutrino masses and the CP asymmetries, the Higgs mass only depends on the flavour charge $a+d$ whereas the false vacuum energy density is determined by a different combination of charges,

$$
m_{S} \simeq \eta^{2(a+d-1)} \frac{v_{\mathrm{EW}}^{2}}{\bar{m}_{\nu}}, \quad \rho_{0}^{1 / 4} \simeq \eta^{2 a+d-1} \frac{v_{\mathrm{EW}}^{2}}{\bar{m}_{\nu}} .
$$

During the tachyonic preheating the energy of the false vacuum is converted mostly into a nonrelativistic gas of $S$ bosons $\left(\left|\vec{p}_{S}\right| / m_{S} \ll 1\right)$, with an admixture of heavy neutrinos. Their contribution to energy density and number densities is determined by their coupling to the Higgs field [28],

$$
\begin{aligned}
& r_{N_{i}}=\frac{\rho_{N_{i}}}{\rho_{0}} \simeq 1.5 \times 10^{-3} g_{N} \lambda f\left(\alpha_{i}, 0.8\right), \\
& n_{N_{i}} \simeq 3.6 \times 10^{-4} g_{N} m_{S}^{3} f(\alpha, 0.8) / \alpha,
\end{aligned}
$$

where $g_{N}=2$ and $f(\alpha, \gamma)=\sqrt{\alpha^{2}+\gamma^{2}}-\gamma$ with $\alpha_{i}=h_{i}^{n} / \sqrt{\lambda}$. For the heaviest neutrinos $N_{2,3}$, one obtains

$$
r_{N_{2,3}} \simeq 1 \times 10^{-3}\left(h_{2}^{n}\right)^{2},
$$

while the relative contribution from the lightest right-handed neutrinos, $r_{N_{1}} / r_{N_{2,3}} \simeq$ $\mathcal{O}\left(\eta^{2}\right)$, is negligible.

For simplicity, we neglect all superpartners as well as $B-L$ gauge bosons and inflaton modes $(T)$ which are produced during tachyonic preheating. We expect their contributions to not significantly change our results, similarly as in supersymmetric leptogenesis [29]. A detailed discussion will be presented in [30]. A further important aspect of tachyonic preheating is the production of cosmic strings [21]. Their effect on the baryon asymmetry is model dependent [31]. It has to be analysed for the parameters of our model [30] taking also into account non-minimal couplings of the inflaton [32].

Our choice of flavour charges implies that the Higgs field $S$ decays exclusively into pairs of $N_{1}$ neutrinos. The resulting decay rate is given by

$$
\Gamma_{S}^{0}=\frac{\left(h_{1}^{n}\right)^{2}}{16 \pi} m_{S}\left[1-\left(2 M_{1} / m_{S}\right)^{2}\right]^{3 / 2} .
$$


Note that if the $S$ boson decayed into more than one heavy neutrino flavour, this would lead to an interplay between lepton asymmetries which could have different signs and it would also change the reheating temperature. In the present paper we restrict our analysis to the simplest case.

\section{$3 \quad$ Entropy production through neutrino decays}

In order to understand the reheating process subsequent to false vacuum decay quantitatively, we have to track the evolution of the following abundancies as functions of the scale factor $a$ : the $S$ Higgs bosons, the heavy $N_{1}$ Majorana neutrinos, the $B-L$ asymmetry, the

standard model radiation $R$ and the gravitinos $\widetilde{G}$. The appropriate tool for this task are the Boltzmann equations in an expanding Friedmann-Lemaitre universe, the formalism of which is summarized in Appendix A. In what follows, we consider comoving number densities,

$$
N_{X}(t)=a(t)^{3} n_{X}(t)
$$

where $a$ is normalized to $a\left(t_{\mathrm{PH}}\right)=1$. This quantity exhibits the advantage of being well defined for times prior to reheating of the universe.

\subsection{Initial conditions}

After tachyonic preheating the universe is filled by a gas of nonrelativistic $S$ bosons as well as heavy $N_{2}$ and $N_{3}$ neutrinos. Given the flavour structure presented in Section 2 , the latter decay into standard model particles on time scales much shorter than the $S$ boson lifetime,

$$
\frac{\Gamma_{N_{2,3}}^{0}}{\Gamma_{S}^{0}} \simeq \eta^{2(a-d-1)} \geq \eta^{-2}, \quad 1 \geq a \geq 0, \quad d \geq 1
$$

Hence, we do not explicitly resolve the time dependence of the $N_{2,3}$ number densities but approximate their evolutions by step functions, i.e. instantaneous drop-offs at times $t_{2}=t_{\mathrm{PH}}+1 / \Gamma_{N_{2}}^{0}$ and $t_{3}=t_{\mathrm{PH}}+1 / \Gamma_{N_{3}}^{0} \simeq t_{2}(\mathrm{cf.} \mathrm{Eq.} 17$ ) $)$,

$$
n_{N_{2,3}}(t) \approx \frac{a\left(t_{\mathrm{PH}}\right)^{3}}{a(t)^{3}} n_{N_{2,3}}\left(t_{\mathrm{PH}}\right) \Theta\left(t_{2}-t\right), \quad t \geq t_{\mathrm{PH}} .
$$

The sudden decay of the $N_{2,3}$ neutrinos sets the stage for the reheating of the universe which is why we choose $t=t_{2} \simeq t_{3}$ as initial time when solving the Boltzmann equations. 
Let us now determine the initial conditions at this time. When the $N_{2,3}$ neutrinos decay they transfer their energy inherited from tachyonic preheating to radiation,

$$
\rho_{R}\left(t_{2}\right)=2 r_{N_{2}} \frac{\rho_{0}}{a^{3}\left(t_{2}\right)} .
$$

For a thermal bath of temperature $T$ the energy and number densities $\rho_{R}$ and $n_{R}$ of radiation quanta are given by

$$
\begin{aligned}
\rho_{R} & =\frac{\pi^{2}}{30} g_{\star, \rho} T^{4}, \\
n_{R} & =\frac{\zeta(3)}{\pi^{2}} g_{\star, n} T^{3},
\end{aligned}
$$

where $g_{\star, \rho}$ and $g_{\star, n}$ denote corresponding effective sums of relativistic degrees of freedom

$$
\begin{aligned}
& g_{\star, \rho}=\sum_{\text {bosons }}\left(T_{i} / T\right)^{4}+\frac{7}{8} \sum_{\text {fermions }}\left(T_{i} / T\right)^{4}, \\
& g_{\star, n}=\sum_{\text {bosons }}\left(T_{i} / T\right)^{3}+\frac{3}{4} \sum_{\text {fermions }}\left(T_{i} / T\right)^{3} .
\end{aligned}
$$

In our numerical analysis we employ the values $g_{\star, \rho}=915 / 4$ and $g_{\star, n}=427 / 2\left(T_{i}=T\right)$ for the minimal supersymmetric standard model (MSSM). Equating the energy densities in Eqs. (26) and 27a yields the initial temperature $T\left(t_{2}\right)$ and thereby the initial comoving number density $N_{R}\left(t_{2}\right)$,

$$
\begin{aligned}
T\left(t_{2}\right) & =\left(\frac{30}{\pi^{2} g_{\star, \rho}} \frac{2 r_{N_{2}} \rho_{0}}{a^{3}\left(t_{2}\right)}\right)^{1 / 4}, \\
N_{R}\left(t_{2}\right) & =a^{3}\left(t_{2}\right) \frac{\zeta(3)}{\pi^{2}} g_{\star, n} T^{3}\left(t_{2}\right) .
\end{aligned}
$$

Note that Eq. (27) provides us with an expression for $T$ as a function of $N_{R}$,

$$
T=\left(\frac{\pi^{2} N_{R}}{\zeta(3) g_{\star, n} a^{3}}\right)^{1 / 3}
$$

As we will argue in Section 3.2.1 this relation can be used to determine the time evolution of the temperature. The out-of-equilibrium decay of $N_{2,3}$ also produces an initial $B-L$ asymmetry ${ }^{4}$ The corresponding comoving number density is given by (cf. Eq. (15))

$$
\begin{aligned}
N_{B-L}\left(t_{2}\right) & =\epsilon_{2} N_{N_{2}}\left(t_{2}\right)+\epsilon_{3} N_{N_{3}}\left(t_{2}\right), \\
& \simeq 0.2 \eta^{-2} \frac{\bar{m}_{\nu} M_{1}}{v_{\mathrm{EW}}^{2}} N_{N_{2}}\left(t_{\mathrm{PH}}\right),
\end{aligned}
$$

\footnotetext{
${ }^{4}$ In Ref. [28] only the initial $B-L$ asymmetry from tachyonic preheating is taken into account.
} 
where the comoving number density $N_{N_{2}}$ at $t_{\mathrm{PH}}$ follows from Eq. (20b).

At $t=t_{2}$ the dominant contribution to the energy density resides in the gas of nonrelativistic $S$ bosons (cf. Eq. (20a)),

$$
\rho_{S}\left(t_{2}\right)=\frac{1}{a^{3}\left(t_{2}\right)}\left(1-2 r_{N_{2}}\right) \rho_{0},
$$

which corresponds to an initial comoving number density

$$
N_{S}\left(t_{2}\right)=a^{3}\left(t_{2}\right) n_{S}\left(t_{2}\right)=a^{3}\left(t_{2}\right) \frac{\rho_{S}\left(t_{2}\right)}{m_{S}} .
$$

According to Eq. A.7), the initial phase space distribution function $f_{S}\left(t_{2}, p\right)$ can be inferred from $n_{S}\left(t_{2}\right)$. Guided by the results of Ref. [28] we make the ansatz of a deltapeaked momentum distribution, i.e. $f_{S}\left(t_{2}, p\right) \propto \delta(p)$, which leads to

$$
f_{S}\left(t_{2}, p\right)=2 \pi^{2} N_{S}\left(t_{2}\right) \frac{\delta(k)}{k^{2}}, \quad k=a\left(t_{2}\right) p\left(t_{2}\right)=a(t) p(t) .
$$

Because of the chosen mass hierarchy $M_{1} \ll m_{S} \simeq M_{2,3}$, the amount of $N_{1}$ neutrinos produced during tachyonic preheating as well as through $S$ decays up to $t_{2}$ is negligibly small. Likewise, in absence of standard model radiation no gravitinos are produced until $t_{2}$,

$$
N_{N_{1}}\left(t_{2}\right)=0, \quad N_{\widetilde{G}}\left(t_{2}\right)=0 .
$$

The time dependence of the scale factor $a(t)$ is governed by the Friedmann equation. For a flat universe and constant equation of state $\omega=\rho / p$ between some time $t_{0}$ and $t$, one has

$$
a(t)=a\left(t_{0}\right)\left[1+\frac{3}{2}(1+\omega)\left(\frac{8 \pi}{3 M_{p}^{2}} \rho_{\text {tot }}\left(t_{0}\right)\right)^{1 / 2}\left(t-t_{0}\right)\right]^{\frac{2}{3(1+\omega)}}
$$

After preheating, until the decay of the $S$ bosons around $t_{S}=t_{2}+1 / \Gamma_{S}^{0}$, the system is mostly matter-dominated. In view of the mass hierarchy $M_{1} / m_{S} \simeq \eta^{2} \ll 1$ the subsequent $S$ decay into relativistic $N_{1}$ neutrinos, however, entails a continuously changing equation of state. We account for that behaviour by working with two constant effective equation of state coefficients $\omega_{2}$ and $\omega_{S}$ for times $t_{2}<t \leq t_{S}$ and $t>t_{S}$, respectively. For times $t_{\mathrm{PH}}<t \leq t_{2}$, we take $\omega=0$. $\omega_{2}$ can be deduced from the decrease in the total energy density until $t_{S}$. Keeping only the leading contributions to $\rho_{\text {tot }}$ one has

$$
\frac{\rho_{S}\left(t_{S}, \omega_{2}\right)+\rho_{N_{1}}\left(t_{S}, \omega_{2}\right)}{\rho_{0} / a\left(t_{2}\right)^{3}}=\left(\frac{a\left(t_{2}\right)}{a\left(t_{S}, \omega_{2}\right)}\right)^{3\left(1+\omega_{2}\right)} .
$$


With explicit expressions for $\rho_{S}$ and $\rho_{N_{1}}$ at hand (cf. Sections 3.2.2 and 3.2.3) this equation can be solved numerically for $\omega_{2}$. Within the region in parameter space to which we restrict our study (cf. Section 2), $\omega_{2}$ typically turns out to be closer to 0 than to $1 / 3$ indicating matter domination. The $\omega_{2}$ mean value, standard deviation, minimum and maximum values are found to be

$$
\omega_{2}=(2.4 \pm 1.6) \times 10^{-2}, \quad \omega_{2}^{\min } \simeq 8.8 \times 10^{-3}, \quad \omega_{2}^{\max } \simeq 8.7 \times 10^{-2} .
$$

At times $t>t_{S}$ most of the initial energy density has already been transferred to $N_{1}$ neutrinos or subsequently to standard model radiation and we can safely use $\omega_{S}=1 / 3$.

\subsection{Boltzmann equations}

Using the conventions introduced in Appendix A we now write down the Boltzmann equations relevant for our specific scenario. In Section 3.2.1 the Boltzmann equations for all species but the gravitino $\left(S, N_{1}, B-L, R\right)$ are given for phase space distribution functions. Subsequently, they are discussed one by one in Sections 3.2.2 through 3.2.5. For the gravitino component we directly give the integrated Boltzmann equation for the comoving number density in Section 3.2.6.

\subsubsection{Collision operators}

The dynamics of our system is dominated by three types of particle interactions: $S$ boson decays into pairs of $N_{1}$ neutrinos, $N_{1}$ neutrino interactions with standard model leptonHiggs pairs $\ell H$ and $\bar{\ell} \bar{H}$ and supersymmetric QCD $2 \rightarrow 2$ scatterings responsible for the production of gravitinos $5^{5}$

The Boltzmann equations for the $S$ bosons and the $N_{1}$ neutrinos take the form

$$
\begin{aligned}
\hat{\mathcal{L}} f_{S} & =C_{S}\left(S \rightarrow N_{1} N_{1}\right), \\
\hat{\mathcal{L}} f_{N_{1}} & =2 C_{N_{1}}\left(S \rightarrow N_{1} N_{1}\right)+C_{N_{1}}\left(N_{1} \leftrightarrow \ell H, \bar{\ell} \bar{H}\right),
\end{aligned}
$$

where the factor of 2 in Eq. (40) accounts for the fact that two $N_{1}$ neutrinos are created per $S$ decay, and $C_{N_{1}}\left(N_{1} \leftrightarrow \ell H, \bar{\ell} \bar{H}\right)$ encompasses $N_{1}$ decays into particles and antiparticles,

$$
C_{N_{1}}\left(N_{1} \leftrightarrow \ell H, \bar{\ell} \bar{H}\right)=C_{N_{1}}\left(N_{1} \leftrightarrow \ell H\right)+C_{N_{1}}\left(N_{1} \leftrightarrow \bar{\ell} \bar{H}\right)
$$

Since we expect their effects to yield only minor corrections, we do not include the rescatterings of $N_{1}$ neutrinos into $S$ bosons as well as scatterings involving massive $Z^{\prime}$ bosons

\footnotetext{
${ }^{5}$ For notation convenience, we refer to $H_{u}$ as $H$ from now on.
} 
which arise in the course of $B-L$ breaking. We have checked that the scatterings $N_{1} \ell \leftrightarrow q \bar{u}, N_{1} \bar{u} \leftrightarrow \ell \bar{q}$ and $N_{1} q \leftrightarrow \ell u$ do not affect the final $B-L$ asymmetry.

The Boltzmann equation for the $B-L$ asymmetry is defined in terms of the respective equations for lepton number $L$ and anti-lepton number $\bar{L}$

$$
\begin{aligned}
\hat{\mathcal{L}} f_{B-L} & =\hat{\mathcal{L}}\left(f_{L}-f_{\bar{L}}\right), \\
\hat{\mathcal{L}} f_{L} & =C_{L}\left(\ell H \leftrightarrow N_{1}\right)+2 C_{L}^{\mathrm{red}}(\ell H \leftrightarrow \bar{\ell} \bar{H}), \\
\hat{\mathcal{L}} f_{\bar{L}} & =C_{\bar{L}}\left(\bar{\ell} \bar{H} \leftrightarrow N_{1}\right)+2 C_{\bar{L}}^{\text {red }}(\bar{\ell} \bar{H} \leftrightarrow \ell H) .
\end{aligned}
$$

The collision operators for decays and inverse decays are able to mimic $\Delta L=2$ scatterings of the type $\ell H \rightleftarrows N_{1} \rightleftarrows \bar{\ell} \bar{H}$ with on-shell $N_{1}$ neutrinos in the $s$-channel. They, however, ignore off-shell scatterings even though these will equally affect the final asymmetry. This leads us to adding reduced collision operators $C_{L}^{\text {red }}$ and $C_{\bar{L}}^{\text {red }}$ to Eqs. (42b) and (42c) that account for the production and decay of off-shell neutrinos, $\ell H \rightleftarrows N_{1}^{*} \rightleftarrows \bar{\ell} \bar{H}$. In Ref. [33] it has been shown that the on- and off-shell contributions to the total CP asymmetry in $\ell H \leftrightarrow \bar{\ell} \bar{H}$ scatterings cancel up to $\mathcal{O}\left(\left(h_{i 1}^{\nu}\right)^{4}\right)$. Hence, one may equivalently say that the reduced collision operators subtract scatterings with real intermediate states.

The temperature $T$ of the thermal bath can be determined as a function of the scale factor from the covariant energy conservation

$$
\dot{\rho}_{\text {tot }}+3 H\left(\rho_{\text {tot }}+p_{\text {tot }}\right)=0 .
$$

After inserting the explicit expressions for the total energy and number densities this relation becomes a non-linear first-order differential equation for $T$. For simplicity, we assume that the energy transfer to the thermal bath happens instantaneously, $\dot{\rho}_{\text {tot }} \approx 0$. Consider a spatial volume $V$ in which $N_{1}$ neutrinos of average energy $\varepsilon_{N_{1}}$ decay into lepton-Higgs pairs. Per decay the energy density of the thermal bath is then increased by $\varepsilon_{N_{1}} / V$ and a new thermal equilibrium at a slightly higher temperature is established right after the decay. According to Eq. 27) the latter entails an increase in $n_{R}$,

$$
n_{R} \rightarrow n_{R}\left(1+\frac{\varepsilon_{N_{1}}}{V \rho_{R}}\right)^{3 / 4} \simeq n_{R}+\frac{3}{4} \frac{\varepsilon_{N_{1}}}{V \rho_{R} / n_{R}}=n_{R}+\frac{r_{R}}{V} .
$$

Therefore, producing two standard model particles adds $r_{R}=3 \varepsilon_{N_{1}} /\left(4 \rho_{R} / n_{R}\right)$ radiation quanta per unit volume $V$ to the thermal bath. This leads us to an effective Boltzmann equation for the number density of radiation

$$
\hat{\mathcal{L}} f_{R}=r_{R}\left(C_{\ell}\left(\ell H \leftrightarrow N_{1}\right)+C_{\bar{\ell}}\left(\bar{\ell} \bar{H} \leftrightarrow N_{1}\right)\right),
$$


where all CP violating contributions have been neglected.

Having formulated the Boltzmann equations for distribution functions we now calculate the various collision operators explicitly and simplify the equations as far as possible.

\subsubsection{Higgs bosons $S$}

In terms of the $S$ decay rate

$$
\Gamma_{S}^{0}=\frac{1}{2 m_{S}} \int d \Pi\left(N_{1}, N_{1}\right)(2 \pi)^{4} \delta^{(4)}\left(\sum p_{\text {out }}-\sum p_{\text {in }}\right)\left|\mathcal{M}\left(S \rightarrow N_{1} N_{1}\right)\right|^{2},
$$

the collision operator for $S$ decay is given by

$$
\hat{\mathcal{L}} f_{S}=C_{S}\left(S \rightarrow N_{1} N_{1}\right)=-\frac{m_{S}}{E_{S}} \Gamma_{S}^{0} f_{S}
$$

This is a linear homogeneous ordinary differential equation which has a unique solution

for each initial value. Given the initial distribution function $f_{S}\left(t_{2}, p\right)$ in Eq. (34) we find

$$
\begin{aligned}
f_{S}(t, p) & =f_{S}\left(t_{2}, p\right) \exp \left[-m_{S} \Gamma_{S}^{0} \int_{t_{2}}^{t} d t^{\prime} E_{S}^{-1}\left(t^{\prime}\right)\right] \\
E_{S}\left(t^{\prime}\right) & =\sqrt{\left(a / a^{\prime}\right)^{2} p^{2}+m_{S}^{2}}, \quad a=a(t), \quad a^{\prime}=a\left(t^{\prime}\right) .
\end{aligned}
$$

Thanks to the momentum delta function in $f_{S}\left(t_{2}, p\right)$ the time integration becomes trivial,

$$
f_{S}(t, p)=f_{S}\left(t_{2}, p\right) e^{-\Gamma_{S}^{0}\left(t-t_{2}\right)}=2 \pi^{2} N_{S}\left(t_{2}\right) \frac{\delta(k)}{k^{2}} e^{-\Gamma_{S}^{0}\left(t-t_{2}\right)}, \quad k=a(t) p .
$$

Hence, the comoving number density $N_{S}$ simply falls off exponentially,

$$
N_{S}(t)=N_{S}\left(t_{2}\right) e^{-\Gamma_{S}^{0}\left(t-t_{2}\right)}
$$

\subsubsection{Heavy Majorana neutrinos $N_{1}$}

The Boltzmann equation for $N_{1}$ neutrinos also involves a collision operator for $S$ decay. Using the tree-level amplitude squared

$$
\left|\mathcal{M}\left(S \rightarrow N_{1} N_{1}\right)\right|^{2}=2\left(h_{1}^{n}\right)^{2} m_{S}^{2}\left[1-\left(2 M_{1} / m_{S}\right)^{2}\right]
$$

the general collision operator in Eq. A.3 takes the form

$$
C_{N_{1}}\left(S \rightarrow N_{1} N_{1}\right)=\frac{\left(h_{1}^{n}\right)^{2} m_{S}^{2}}{2 E_{N_{1}}}\left[1-\left(2 M_{1} / m_{S}\right)^{2}\right] \int d \Pi\left(N_{1} \mid N_{1} ; S\right)(2 \pi)^{4} \delta^{(4)} f_{S} .
$$


With the explicit expression for $f_{S}$ in Eq. (50) the phase space integration becomes

$$
\int d \Pi\left(N_{1} \mid N_{1} ; S\right)(2 \pi)^{4} \delta^{(4)} f_{S}=\frac{1}{a^{3}} \frac{\pi N_{S}}{8 m_{S} E_{N_{1}}} \delta\left(E_{N_{1}}-m_{S} / 2\right),
$$

where a symmetry factor $1 / 2$ results from the fact that two $N_{1}$ neutrinos are involved in the decay process. Employing the result Eq. (22) for the $S$ decay width we obtain for the collision operator

$$
C_{N_{1}}\left(S \rightarrow N_{1} N_{1}\right)=\frac{1}{a^{3}} \frac{\pi^{2} N_{S} \Gamma_{S}^{0}}{E_{N_{1}}^{2}}\left[1-\left(2 M_{1} / m_{S}\right)^{2}\right]^{-1 / 2} \delta\left(E_{N_{1}}-m_{S} / 2\right) .
$$

The collision operator for $N_{1}$ decay into standard model particles has the familiar form

$$
C_{N_{1}}\left(N_{1} \leftrightarrow \ell H, \bar{\ell} \bar{H}\right)=-\frac{M_{1}}{E_{N_{1}}} \Gamma_{N_{1}}^{0}\left(f_{N_{1}}-f_{N_{1}}^{\mathrm{eq}}\right)
$$

In total, the Boltzmann equation for $N_{1}$ neutrinos encompasses two production and one decay term. On the one hand, the collision operator in Eq. (55) and the term proportional to $f_{N_{1}}^{\text {eq }}$ in Eq. (56) represent $N_{1}$ production from $S$ decay and from inverse decays in the thermal bath, respectively. On the other hand, the term proportional to $f_{N_{1}}$ in Eq. (56) accounts for $N_{1}$ decays. It is convenient to decompose the $N_{1}$ population into two independently evolving components: Nonthermal neutrinos $N_{1}^{S}$ stemming from $S$ decays and thermal neutrinos $N_{1}^{T}$ originating from the thermal bath. The respective Boltzmann equations are then given as

$$
\begin{aligned}
\hat{\mathcal{L}} f_{N_{1}}^{S} & =-\frac{M_{1}}{E_{N_{1}}} \Gamma_{N_{1}}^{0} f_{N_{1}}^{S}+2 \frac{1}{a^{3}} \frac{\pi^{2} N_{S} \Gamma_{S}^{0}}{E_{N_{1}}^{2}}\left[1-\left(2 M_{1} / m_{S}\right)^{2}\right]^{-1 / 2} \delta\left(E_{N_{1}}-m_{S} / 2\right), \\
\hat{\mathcal{L}} f_{N_{1}}^{T} & =-\frac{M_{1}}{E_{N_{1}}} \Gamma_{N_{1}}^{0} f_{N_{1}}^{T}+\frac{M_{1}}{E_{N_{1}}} \Gamma_{N_{1}}^{0} f_{N_{1}}^{\mathrm{eq}} .
\end{aligned}
$$

The sum of the nonthermal and thermal distribution functions yields the total $N_{1}$ distribution function, $f_{N_{1}}=f_{N_{1}}^{S}+f_{N_{1}}^{T}$.

The Boltzmann equation for the nonthermal neutrinos $N_{1}^{S}$ can be solved exactly. Starting from zero initial abundance we find

$$
\begin{aligned}
f_{N_{1}}^{S}(t, p)= & 2 \pi^{2} \Gamma_{S}^{0}\left[1-\left(2 M_{1} / m_{S}\right)^{2}\right]^{-1 / 2} \int_{t_{2}}^{t} d t^{\prime}\left[\delta\left(E_{N_{1}}\left(t^{\prime}\right)-m_{S} / 2\right)\right. \\
& \left.\times \frac{N_{S}\left(t^{\prime}\right)}{a^{\prime 3} E_{N_{1}}^{2}\left(t^{\prime}\right)} \exp \left(-M_{1} \Gamma_{N_{1}}^{0} \int_{t^{\prime}}^{t} d t^{\prime \prime} E_{N_{1}}^{-1}\left(t^{\prime \prime}\right)\right)\right]
\end{aligned}
$$


where $E_{N_{1}}\left(t^{\prime}\right)$ is defined analogously to $E_{S}\left(t^{\prime}\right)$ in Eq. (49). The energies $E_{N_{1}}$ therefore redshift as

$$
E_{N_{1}}(t)=E_{N_{1}}\left(t^{\prime}\right) \frac{a^{\prime}}{a}\left[1+\left(\left(\frac{a}{a^{\prime}}\right)^{2}-1\right)\left(\frac{M_{1}}{E_{N_{1}}\left(t^{\prime}\right)}\right)^{2}\right]^{1 / 2} .
$$

If we evaluate this relation with $E_{N_{1}}\left(t^{\prime}\right)=m_{S} / 2$ we obtain the redshifted energy at time $t$ of a neutrino $N_{1}$ that has been produced in $S$ decay at time $t^{\prime}$. Let us denote this quantity by $\mathcal{E}_{N_{1}}\left(t^{\prime}, t\right)$,

$$
\mathcal{E}_{N_{1}}\left(t^{\prime}, t\right)=\frac{m_{S}}{2} \frac{a^{\prime}}{a}\left[1+\left(\left(\frac{a}{a^{\prime}}\right)^{2}-1\right)\left(\frac{2 M_{1}}{m_{S}}\right)^{2}\right]^{1 / 2} .
$$

The energy delta function in the integrand of Eq. (58) thus turns $E_{N_{1}}\left(t^{\prime \prime}\right)$ into $\mathcal{E}_{N_{1}}\left(t^{\prime}, t^{\prime \prime}\right)$. Meanwhile, it can be rewritten as a function of $E_{N_{1}}(t)$, the energy at time $t$,

$$
\delta\left(E_{N_{1}}\left(t^{\prime}\right)-m_{S} / 2\right)=\left(\frac{a^{\prime}}{a}\right)^{2} \frac{m_{S} / 2}{\mathcal{E}_{N_{1}}\left(t^{\prime}, t\right)} \delta\left(E_{N_{1}}(t)-\mathcal{E}_{N_{1}}\left(t^{\prime}, t\right)\right) .
$$

The final result for $f_{N_{1}}^{S}$ then reads

$$
\begin{aligned}
f_{N_{1}}^{S}(t, p)= & \frac{1}{a^{3}} 2 \pi^{2} \Gamma_{S}^{0}\left[\left(m_{S} / 2\right)^{2}-M_{1}^{2}\right]^{-1 / 2} \int_{t_{2}}^{t} d t^{\prime}\left[\frac{a}{a^{\prime}} \delta\left(E_{N_{1}}(t)-\mathcal{E}_{N_{1}}\left(t^{\prime}, t\right)\right)\right. \\
& \left.\times \frac{N_{S}\left(t^{\prime}\right)}{\mathcal{E}_{N_{1}}\left(t^{\prime}, t\right)} \exp \left(-M_{1} \Gamma_{N_{1}}^{0} \int_{t^{\prime}}^{t} d t^{\prime \prime} \mathcal{E}_{N_{1}}^{-1}\left(t^{\prime}, t^{\prime \prime}\right)\right)\right] .
\end{aligned}
$$

By integrating over the $N_{1}^{S}$ phase space, we obtain the number density $n_{N_{1}}^{S}$ of the nonthermal neutrinos

$$
\begin{aligned}
n_{N_{1}}^{S} & =g_{N_{1}} \int \frac{d^{3} p}{(2 \pi)^{3}} f_{N_{1}}^{S}=\frac{2 \Gamma_{S}^{0}}{a^{3}} \int_{t_{2}}^{t} d t^{\prime}\left[N_{S}\left(t^{\prime}\right) \exp \left(-M_{1} \Gamma_{N_{1}}^{0} \int_{t^{\prime}}^{t} d t^{\prime \prime} \mathcal{E}_{N_{1}}^{-1}\left(t^{\prime}, t^{\prime \prime}\right)\right)\right] \\
& =\int_{t_{2}}^{t} d t^{\prime} \delta n_{N_{1}}^{S}\left(t^{\prime}, t\right) .
\end{aligned}
$$

The corresponding results for the energy and interaction densities $\rho_{N_{1}}^{S}$ and $\gamma_{N_{1}}^{S}=\gamma\left(N_{1}^{S} \rightarrow\right.$ 
$\ell H, \bar{\ell} \bar{H})$ can conveniently be expressed using $\delta n_{N_{1}}^{S}\left(t, t^{\prime}\right)$ introduced in Eq. 63b,

$$
\begin{aligned}
& \rho_{N_{1}}^{S}=\int_{t_{2}}^{t} d t^{\prime} \mathcal{E}_{N_{1}}\left(t^{\prime}, t\right) \delta n_{N_{1}}^{S}\left(t^{\prime}, t\right), \\
& \gamma_{N_{1}}^{S}=\int_{t_{2}}^{t} d t^{\prime} \frac{M_{1}}{\mathcal{E}_{N_{1}}\left(t^{\prime}, t\right)} \Gamma_{N_{1}}^{0} \delta n_{N_{1}}^{S}\left(t^{\prime}, t\right)=n_{N_{1}}^{S} \Gamma_{N_{1}}^{S},
\end{aligned}
$$

where $\Gamma_{N_{1}}^{S}$ denotes the $N_{1}$ decay width weighted with the average inverse time dilatation factor for nonthermal neutrinos

$$
\Gamma_{N_{1}}^{S}=\left\langle\frac{M_{1}}{E_{N_{1}}}\right\rangle_{S} \Gamma_{N_{1}}^{0}=\frac{1}{n_{N_{1}}^{S}} \int_{t_{2}}^{t} d t^{\prime} \frac{M_{1}}{\mathcal{E}_{N_{1}}\left(t^{\prime}, t\right)} \delta n_{N_{1}}^{S}\left(t^{\prime}, t\right) \Gamma_{N_{1}}^{0} .
$$

The exact phase space distribution function $f_{N_{1}}^{T}$ for thermal neutrinos $N_{1}^{T}$ is given as the unique solution of Eq. (57b) for the initial distribution $f_{N_{1}}^{T}\left(t_{2}, p\right)=0$,

$$
f_{N_{1}}^{T}(t, p)=\int_{t_{2}}^{t} d t^{\prime} \exp \left(-M_{1} \Gamma_{N_{1}}^{0} \int_{t^{\prime}}^{t} d t^{\prime \prime} E_{N_{1}}^{-1}\left(t^{\prime \prime}\right)\right) \frac{M_{1}}{E_{N_{1}}\left(t^{\prime}\right)} \Gamma_{N_{1}}^{0} f_{N_{1}}^{\mathrm{eq}}\left(t^{\prime}, p\right) .
$$

As the thermal neutrinos are produced within a broad range of energies, it cannot be integrated over phase space as simply as in the nonthermal case. However, since the thermal neutrinos inherit their momentum distribution from the thermal bath it is reasonable to assume that they are approximately in kinetic equilibrium,

$$
f_{N_{1}}^{T}(t, p) \approx \frac{N_{N_{1}}^{T}}{N_{N_{1}}^{\mathrm{eq}}} f_{N_{1}}^{\mathrm{eq}}(t, p), \quad f_{N_{1}}^{\mathrm{eq}}(t, p)=e^{-E_{N_{1}} / T}
$$

This approximation holds if the quotient $f_{N_{1}}^{T} / f_{N_{1}}^{\text {eq }}$, with $f_{N_{1}}^{T}$ taken from Eq. (67), is independent of the neutrino momentum $p$. In Appendix B we will demonstrate numerically in the context of a specific parameter example that this can usually be assumed to be the case in our scenario. Under the assumption of kinetic equilibrium the comoving number density $N_{N_{1}}^{T}$ is the unique solution of the integrated Boltzmann equation

$$
a H \frac{d}{d a} N_{N_{1}}^{T}=-\left(N_{N_{1}}^{T}-N_{N_{1}}^{\mathrm{eq}}\right) \Gamma_{N_{1}}^{T} .
$$

Here, $\Gamma_{N_{1}}^{T}$ stands for the $N_{1}$ decay width weighted with the average inverse time dilatation factor for thermal neutrinos

$$
\Gamma_{N_{1}}^{T}=\left\langle\frac{M_{1}}{E_{N_{1}}}\right\rangle_{T} \Gamma_{N_{1}}^{0}=\frac{K_{1}(z)}{K_{2}(z)} \Gamma_{N_{1}}^{0}
$$


where $z=M_{1} / T$, and $K_{1,2}(z)$ are modified Bessel functions of the second kind. Note that

$$
\begin{aligned}
& \gamma_{N_{1}}^{T}=\gamma\left(N_{1}^{T} \rightarrow \ell H, \bar{\ell} \bar{H}\right)=n_{N_{1}}^{T} \Gamma_{N_{1}}^{T}, \\
& \gamma_{N_{1}}^{\text {eq }}=\gamma^{\text {eq }}\left(N_{1} \rightarrow \ell H, \bar{\ell} \bar{H}\right)=n_{N_{1}}^{\text {eq }} \Gamma_{N_{1}}^{T}
\end{aligned}
$$

\subsection{4 $\quad B-L$ asymmetry}

The collision operators for decays and inverse decays present themselves as

$$
\begin{aligned}
C_{L}\left(\ell H \leftrightarrow N_{1}\right) & \sim f_{N_{1}}\left|\mathcal{M}\left(N_{1} \rightarrow \ell H\right)\right|^{2}-f_{H} f_{\ell}\left|\mathcal{M}\left(\ell H \rightarrow N_{1}\right)\right|^{2}, \\
C_{\bar{L}}\left(\bar{\ell} \bar{H} \leftrightarrow N_{1}\right) & \sim f_{N_{1}}\left|\mathcal{M}\left(N_{1} \rightarrow \bar{\ell} \bar{H}\right)\right|^{2}-f_{\bar{H}} f_{\bar{\ell}}\left|\mathcal{M}\left(\bar{\ell} \bar{H} \rightarrow N_{1}\right)\right|^{2} .
\end{aligned}
$$

Using the definition of the $\mathrm{CP}$ parameter $\epsilon_{1}$ and $\mathrm{CPT}$ invariance, the various partial amplitudes squared are related to the total amplitude squared as follows

$$
\begin{aligned}
\left|\mathcal{M}\left(N_{1} \rightarrow \ell H\right)\right|^{2} & =\left|\mathcal{M}\left(\bar{\ell} \bar{H} \rightarrow N_{1}\right)\right|^{2}=\frac{1}{2}\left(1+\epsilon_{1}\right)\left|\mathcal{M}_{N_{1}}\right|^{2}, \\
\left|\mathcal{M}\left(N_{1} \rightarrow \bar{\ell} \bar{H}\right)\right|^{2} & =\left|\mathcal{M}\left(\ell H \rightarrow N_{1}\right)\right|^{2}=\frac{1}{2}\left(1-\epsilon_{1}\right)\left|\mathcal{M}_{N_{1}}\right|^{2},
\end{aligned}
$$

where, at tree-level, $\left|\mathcal{M}_{N_{1}}\right|^{2}$ is given as

$$
\left|\mathcal{M}_{N_{1}}\right|^{2}=\left|\mathcal{M}\left(N_{1} \rightarrow \ell H, \bar{\ell} \bar{H}\right)\right|^{2}=4\left(h^{\nu \dagger} h^{\nu}\right)_{11} M_{1}^{2}
$$

The reduced collision operators in Eq. (42) account for the production of off-shell neutrinos $N_{1}^{*}$ which subsequently decay into the CP conjugate of the lepton-Higgs pair from which they were produced. Working up to leading order in $\epsilon_{1}$ we may take the decays $N_{1}^{*} \rightarrow \ell H, \bar{\ell} \bar{H}$ to equally branch into particles and antiparticles

$$
C_{L, \bar{L}}^{\mathrm{red}}(\ell H \leftrightarrow \bar{\ell} \bar{H}) \sim \pm\left[f_{\bar{\ell}} f_{\bar{H}} \cdot \frac{1}{2}\left|\mathcal{M}\left(\bar{\ell} \bar{H} \rightarrow N_{1}^{*}\right)\right|^{2}-f_{\ell} f_{H} \cdot \frac{1}{2}\left|\mathcal{M}\left(\ell H \rightarrow N_{1}^{*}\right)\right|^{2}\right] .
$$

For $M_{1} \ll 10^{14} \mathrm{GeV}$ the $\mathrm{CP}$ preserving parts of the off-shell scatterings are negligibly small [34]. We thus discard them keeping only the $\mathrm{CP}$ violating contributions to the reduced collision operators. Imposing that the total CP asymmetry of lepton-Higgs scatterings be zero up to $\mathcal{O}\left(\left(h_{i 1}^{\nu}\right)^{4}\right)$, we deduce

$$
\left|\mathcal{M}\left(\ell H \rightarrow N_{1}^{*}\right)\right|^{2}=-\frac{1}{2}\left(-\epsilon_{1}\right)\left|\mathcal{M}_{N_{1}}\right|^{2}, \quad\left|\mathcal{M}\left(\bar{\ell} \bar{H} \rightarrow N_{1}^{*}\right)\right|^{2}=-\frac{1}{2}\left(+\epsilon_{1}\right)\left|\mathcal{M}_{N_{1}}\right|^{2} .
$$


The above results allow us to write all collision operators as integrals over the total amplitude squared $\left|\mathcal{M}_{N_{1}}\right|^{2}$. Assuming kinetic equilibrium for leptons and antileptons as well as thermal equilibrium for all other standard model particles, we obtain

$$
\hat{\mathcal{L}} f_{B-L}=\frac{1}{2 g_{\ell} p} \int d \Pi\left(\ell \mid H ; N_{1}\right)(2 \pi)^{4} \delta^{(4)} \frac{1}{2}\left|\mathcal{M}_{N_{1}}\right|^{2}\left[2 \epsilon_{1}\left(f_{N_{1}}-f_{N_{1}}^{\mathrm{eq}}\right)-\frac{N_{B-L}}{N_{\ell}^{\mathrm{eq}}} f_{N_{1}}^{\mathrm{eq}}\right] .
$$

As for the $N_{1}$ Boltzmann equation, we split the $N_{1}$ distribution function into its thermal and nonthermal parts. After integrating over phase space we retrieve the interaction densities $\gamma_{N_{1}}^{S}, \gamma_{N_{1}}^{T}$ and $\gamma_{N_{1}}^{\text {eq }}$ (cf. Eq. A.8) ),

$$
a H \frac{d}{d a} N_{B-L}=a^{3}\left[\epsilon_{1}\left(\gamma_{N_{1}}^{S}+\gamma_{N_{1}}^{T}-\gamma_{N_{1}}^{\mathrm{eq}}\right)-\frac{N_{B-L}}{2 N_{\ell}^{\mathrm{eq}}} \gamma_{N_{1}}^{\mathrm{eq}}\right] .
$$

In terms of comoving number densities and averaged decay rates this Boltzmann equation then reads

$$
a H \frac{d}{d a} N_{B-L}=\epsilon_{1} N_{N_{1}}^{S} \Gamma_{N_{1}}^{S}+\epsilon_{1}\left(N_{N_{1}}^{T}-N_{N_{1}}^{\mathrm{eq}}\right) \Gamma_{N_{1}}^{T}-\frac{N_{N_{1}}^{\mathrm{eq}}}{2 N_{\ell}^{\mathrm{eq}}} \Gamma_{N_{1}}^{T} N_{B-L} .
$$

Similarly to the $N_{1}$ abundance we also split the $B-L$ asymmetry into two components: A nonthermal asymmetry $N_{B-L}^{S}$ produced in $N_{1}^{S}$ decays and a thermal asymmetry $N_{B-L}^{T}$ generated from the thermal bath,

$$
\begin{aligned}
& a H \frac{d}{d a} N_{B-L}^{S}=\epsilon_{1} N_{N_{1}}^{S} \Gamma_{N_{1}}^{S}-\frac{N_{N_{1}}^{\mathrm{eq}}}{2 N_{\ell}^{\mathrm{eq}}} \Gamma_{N_{1}}^{T} N_{B-L}^{S}, \\
& a H \frac{d}{d a} N_{B-L}^{T}=\epsilon_{1}\left(N_{N_{1}}^{T}-N_{N_{1}}^{\mathrm{eq}}\right) \Gamma_{N_{1}}^{T}-\frac{N_{N_{1}}^{\mathrm{eq}}}{2 N_{\ell}^{\mathrm{eq}}} \Gamma_{N_{1}}^{T} N_{B-L}^{T} .
\end{aligned}
$$

A comparison of the corresponding final baryon asymmetries $\eta_{B}^{S}$ and $\eta_{B}^{T}$ will allow us to identify the relative importance of nonthermal and thermal leptogenesis in different regions of the parameter space (cf. Section 5).

\subsubsection{Radiation $R$}

In order to obtain an effective Boltzmann equation for the number density of radiation quanta we add up the contributions coming from decays into standard model particles and antiparticles. Neglecting any CP violating effects Eq. 45) can be written as

$$
\hat{\mathcal{L}} f_{R}=r_{R} \frac{1}{2 g_{\ell} p} \int d \Pi\left(\ell \mid H ; N_{1}\right)(2 \pi)^{4} \delta^{(4)} \frac{1}{2}\left|\mathcal{M}_{N_{1}}\right|^{2}\left[2 f_{N_{1}}-2 f_{N_{1}}^{\text {eq }}\right] .
$$


Splitting $f_{N_{1}}$ into its thermal and nonthermal parts and integrating over phase space, one gets

$$
a H \frac{d}{d a} N_{R}=r_{R}^{S} N_{N_{1}}^{S} \Gamma_{N_{1}}^{S}+r_{R}^{T}\left(N_{N_{1}}^{T}-N_{N_{1}}^{\mathrm{eq}}\right) \Gamma_{N_{1}}^{T} .
$$

Since the two different sorts of $N_{1}$ neutrinos possess different average energies, two independent factors $r_{R}^{S}$ and $r_{R}^{T}$ have been introduced in order to keep track of the radiation quanta produced in the decays of nonthermal and thermal neutrinos respectively,

$$
r_{R}^{S}=\frac{3 \varepsilon_{N_{1}}^{S}}{4 \varepsilon_{R}}, \quad r_{R}^{T}=\frac{3 \varepsilon_{N_{1}}^{T}}{4 \varepsilon_{R}} .
$$

The average energies per (non-)thermal neutrino as well as the average energy per radiation quantum are obtained from the respective ratios of energy and number densities (cf. Eqs. (27), (63) and (64),

$$
\varepsilon_{R}=\rho_{R} / n_{R}, \quad \varepsilon_{N_{1}}^{S}=\rho_{N_{1}}^{S} / n_{N_{1}}^{S}, \quad \varepsilon_{N_{1}}^{T}=\rho_{N_{1}}^{T} / n_{N_{1}}^{T}=3 T+\frac{K_{1}(z)}{K_{2}(z)} M_{1} .
$$

$r_{R}^{S}$ and $r_{R}^{T}$ clearly depend on the temperature $T$ which, in turn, is deduced from the radiation number density $N_{R}$ according to Eq. (30).

\subsubsection{Gravitinos $\widetilde{G}$}

Gravitinos are produced through scattering processes in the thermal bath. The evolution of their comoving number density is governed by the Boltzmann equation

$$
a H \frac{d}{d a} N_{\widetilde{G}}=a^{3} \gamma_{\widetilde{G}}(T) .
$$

The dominant contribution to $\gamma_{\widetilde{G}}$ comes from QCD scatterings. For supersymmetric QCD and up to leading order in the strong gauge coupling $g_{s}$, one has [15]

$$
\gamma_{\widetilde{G}}(T)=\left(1+\frac{m_{\tilde{g}}^{2}(T)}{3 m_{\widetilde{G}}^{2}}\right) \frac{54 \zeta(3) g_{s}^{2}(T)}{\pi^{2} M_{p}^{2}} T^{6}\left[\ln \left(\frac{T^{2}}{m_{g}^{2}(T)}\right)+0.8846\right] .
$$

Here, $m_{\tilde{g}}$ denotes the energy scale-dependent gluino mass and $m_{g}$ is the gluon plasma mass,

$$
m_{\tilde{g}}(T)=\frac{g_{s}^{2}(T)}{g_{s}^{2}\left(\mu_{0}\right)} m_{\tilde{g}}\left(\mu_{0}\right), \quad m_{g}(T)=\sqrt{3 / 2} g_{s}(T) T,
$$




\begin{tabular}{ccccccc} 
Point & Label & $v_{B-L}[\mathrm{GeV}]$ & $M_{1}[\mathrm{GeV}]$ & $\widetilde{m}_{1}[\mathrm{eV}]$ & $m_{\widetilde{G}}[\mathrm{GeV}]$ & $m_{\tilde{g}}[\mathrm{GeV}]$ \\
\hline \hline Section 4 & Red circle & $5.8 \times 10^{13}$ & $1.4 \times 10^{10}$ & $3 \times 10^{-3}$ & 100 & 800 \\
Ref. [3] & White circle & $3.0 \times 10^{12}$ & $1.0 \times 10^{10}$ & $1 \times 10^{-3}$ & 100 & 800 \\
\hline \hline
\end{tabular}

Table 2: Values of the input parameters chosen for the discussion in Section 44 and Ref. [3], respectively. In Figs. 5, 6, 7, 8, 10, 13 and 14 in Section 5 and Appendices $C$ and $D$ the positions of both parameter points in parameter space are marked with different labels as indicated.

where we choose the $Z$ boson mass $M_{Z}$ as reference scale $\mu_{0}$. The scale dependence of $g_{s}$ is dictated by the corresponding MSSM renormalization group equation

$$
g_{s}(\mu)=g_{s}\left(\mu_{0}\right)\left[1+\frac{3}{8 \pi^{2}} g_{s}\left(\mu_{0}\right)^{2} \ln \left(\mu / \mu_{0}\right)\right]^{-1 / 2},
$$

with $\mu$ being the typical energy scale during reheating. It can be estimated by the average energy per relativistic particle in the bath: $\mu \simeq \varepsilon_{R} \simeq 3 T$. For instance, at temperatures $T=10^{8}, 10^{10}, 10^{12} \mathrm{GeV}$ the strong gauge coupling takes on values $g_{s}=0.90,0.84,0.80$. The gravitino mass $m_{\widetilde{G}}$ and the low-scale gluino mass $m_{\tilde{g}}\left(\mu_{0}\right)$ remain as free parameters.

\section{An illustrative example}

In the previous section we have seen how the decay of the false vacuum of unbroken $B-L$ symmetry generates - via production and decay of heavy neutrinos - entropy, baryon asymmetry and dark matter. We have numerically solved this inital-value problem by means of Boltzmann equations, with the initial conditions described in Section 3.1. Before we turn to a detailed discussion of the parameter space we first describe, as an example, one solution for a representative choice of parameters. A similar study, albeit not as detailed, has already been performed in Ref. [3]. The values for the input parameters chosen in this section as well as in Ref. [3] are outlined in Tab. 2. In the present case, the selected values for $v_{B-L}$ and $M_{1}$ correspond to Froggatt-Nielsen charges of $a=1 / 2$ and $d \simeq 1.5$. Note that we have adjusted $M_{1}$ such that we obtain the right gravitino abundance for dark matter. The input parameters in Tab. 2 directly determine a couple of further important parameters

$$
m_{S}, M_{2,3} \simeq 4.1 \times 10^{12} \mathrm{GeV}, \lambda \simeq 5.0 \times 10^{-3}, \epsilon_{1} \simeq 1.4 \times 10^{-6}, \epsilon_{2,3} \simeq-4.1 \times 10^{-4} \text {. }
$$

We have chosen opposite signs for the CP asymmetries $\epsilon_{1}$ and $\epsilon_{2,3}$, so that one can easily distinguish their respective contributions to the final $B-L$ asymmetry. 

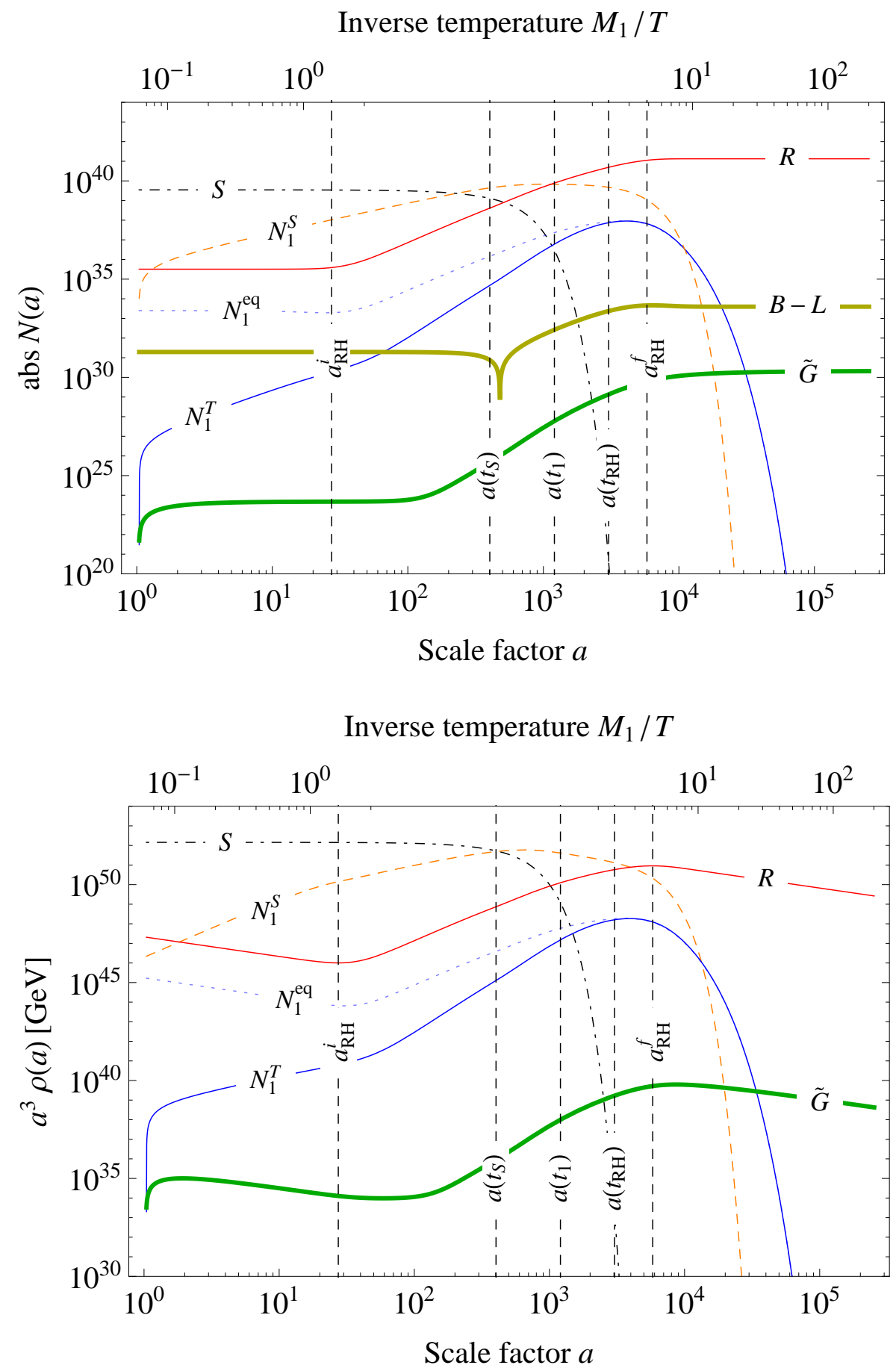

Figure 1: Comoving number (upper panel) and energy (lower panel) densities for $S$ bosons, $N_{1}$ neutrinos produced in $S$ decays $\left(N_{1}^{S}\right)$, thermally produced $N_{1}$ neutrinos $\left(N_{1}^{T}\right), N_{1}$ neutrinos in thermal equilibrium ( $N_{1}^{\mathrm{eq}}$, for comparison), radiation $(R), B-L$ charge, and gravitinos $(\widetilde{G})$ as functions of the scale factor $a$. The corresponding values of the model parameters are given in Tab. 2 . 


\subsection{Comoving number and energy densities}

The evolution with the scale factor $a$ of the comoving number densities and the various components of the energy density are presented in Fig. 1. Both plots start at the time of $N_{2,3}$ decay, $a\left(t_{2}\right) \simeq 1.04$, and end at a final scale factor of $a_{f} \simeq 2.53 \times 10^{5}$. The values of the scale factor corresponding to $S$ and $N_{1}$ decays as well as to reheating (cf. Appendix C) are also indicated

$$
a\left(t_{S}=t_{2}+1 / \Gamma_{S}^{0}\right) \simeq 400, \quad a\left(t_{1}=t_{S}+1 / \Gamma_{N_{1}}^{0}\right) \simeq 1200, \quad a\left(t_{R H}\right) \simeq 3000 .
$$

Tachyonic preheating results in an initial state at $t=t_{2}$ that mainly consists of $S$ bosons and to a smaller degree standard model radiation that stems from the decay of the $N_{2,3}$ neutrinos, $\rho_{R}\left(t_{2}\right) / \rho_{S}\left(t_{2}\right) \sim 10^{-5}$, and which inherits an initial $B-L$ asymmetry equivalent to $\eta_{B} \simeq-3.9 \times 10^{-5}$. Around $t=t_{S}$ the $S$ bosons decay into relativistic and nonthermal $N_{1}$ neutrinos. Their subsequent decay into standard model particles then washes out the initial (negative) asymmetry, builds up a new (positive) asymmetry and leads to the production of the main part of the radiation. The energy transfer to the thermal bath, i.e. the process of reheating, takes place between $a_{R H}^{i} \simeq 27$ and $a_{R H}^{f} \simeq 5800$. At these values of the scale factor the derivative of the comoving radiation energy density $a^{3} \rho_{R}$ vanishes. Meanwhile, thermal neutrinos and gravitinos are continuously produced in the thermal bath. As both species inherit their momentum distributions from the bath, they are always in approximate kinetic equilibrium (cf. Appendix B). From $a \simeq 4100$ onwards the number density of the thermal $N_{1}$ neutrinos exceeds the thermal equilibrium abundance. At $a \sim 10^{5}$ the $B-L$ asymmetry and the gravitino abundance have reached at their final values.

\subsection{Baryon asymmetry}

The present value of the baryon asymmetry as well as its nonthermal and thermal contributions are obtained from

$$
\eta_{B}=\frac{n_{B}^{0}}{n_{\gamma}^{0}}=\eta_{B}^{S}+\eta_{B}^{T}, \quad \eta_{B}^{S, T}=\left.c_{\mathrm{sph}} \frac{g_{\star, s}^{0}}{g_{\star, s}} \frac{N_{B-L}^{S, T}}{N_{\gamma}}\right|_{a_{f}} .
$$

In the supersymmetric standard model the sphaleron conversion factor is $c_{\mathrm{sph}}=8 / 23$, the effective number of degrees of freedom at high and low temperatures is $g_{\star, s}=915 / 4$ and $g_{\star, s}^{0}=43 / 11$, respectively, and the number density of photons is $N_{\gamma}=a^{3} g_{\gamma} \zeta(3) / \pi^{2} T^{3}$. For our choice of parameters we obtain the asymmetries

$$
\eta_{B} \simeq 1.9 \times 10^{-8}, \quad \eta_{B}^{S} \simeq 1.9 \times 10^{-8}, \quad \eta_{B}^{T} \simeq 2.8 \times 10^{-10} .
$$


The calculated baryon asymmetry is larger than the observed one, $\eta_{B}^{\text {obs }} \simeq 6.2 \times 10^{-10}[20]$, by about a factor 30. This is consistent since $\epsilon_{1}$ is an estimate for the maximal CP asymmetry. We find that $\eta_{B}$ is dominated by the nonthermal contribution due to $N_{1}^{S}$ decays, $\eta_{B}^{S} \simeq \eta_{B}$. The contribution from thermal neutrinos, even though it reaches the right order of magnitude, slightly falls short of the observed value.

Let us emphasize that given the choice of parameters in Tab. 2 standard thermal leptogenesis, with a given thermal bath, is able to produce the right amount of baryon asymmetry. Using a final efficiency factor of $\kappa_{f}\left(\widetilde{m}_{1}\right) \simeq 0.1$, one obtains (cf. [33])

$$
\eta_{B}^{\mathrm{th}}=\frac{3}{4} \frac{g_{*, s}^{0}}{g_{*, s}} c_{\mathrm{sph}} \epsilon_{1} \kappa_{f}\left(\widetilde{m}_{1}\right) \simeq 6 \times 10^{-10} .
$$

In the case under study $\eta_{B}^{T}$ turns out to be smaller than $\eta_{B}^{\text {th }}$ roughly by a factor of 2 because the entropy production during $N_{1}^{S}$ decay enhances the washout rate due to inverse $N_{1}$ decays.

The evolution of the nonthermal and thermal lepton asymmetries is controlled by three different interaction rates which enter the Boltzmann equations 80a and 80b

$$
\Gamma_{B-L}^{S}=\epsilon_{1} \frac{N_{N_{1}}^{S}}{N_{B-L}^{S}} \Gamma_{N_{1}}^{S}, \quad \Gamma_{B-L}^{T}=\epsilon_{1} \frac{N_{N_{1}}^{T}-N_{N_{1}}^{\mathrm{eq}}}{N_{B-L}^{T}} \Gamma_{N_{1}}^{T}, \quad \Gamma_{W}=\frac{N_{N_{1}}^{\mathrm{eq}}}{2 N_{\ell}^{\mathrm{eq}}} \Gamma_{N_{1}}^{T} .
$$

They account for the decay of nonthermal neutrinos, the decay of thermal neutrinos and the washout effects due to inverse neutrino decays, respectively. Their relative importance as well as their influence on the generation of the asymmetries are illustrated in Fig. 2 . The respective interactions become efficient once the corresponding rates are of the same order as the Hubble rate or larger. This is why it takes until $a_{1} \simeq 210$, when $\Gamma_{B-L}^{S} / H \gtrsim$ $\mathcal{O}(1)$ for the first time, for $N_{B-L}^{S}$ to begin to increase. At $a_{2} \simeq 480$ the initial negative asymmetry has been compensated by the generated positive one, and $N_{B-L}^{S}$ changes sign. Subsequently, for scale factors around $a\left(t_{1}\right)$, the ratio $\Gamma_{B-L}^{S} / H$ remains approximately constant leading to the generation of the main part of the asymmetry. Meanwhile, due to the continuous entropy production from nonthermal neutrino decays, the washout processes gain in importance. At $a_{3} \simeq 1500$ the rate $\Gamma_{W}$ becomes comparable to $H$, which is reflected in a slight decrease of the slope of $N_{B-L}^{S}$. From $a_{7} \simeq 6300$ onwards, which is shortly after $\rho_{R}=\rho_{N_{1}}^{S}$, the washout even dominates over the asymmetry production from $N_{1}^{S}$ decays. Hence, the maximal nonthermal asymmetry reached at $a_{7}$ is slightly washed out until it eventually freezes out when $\Gamma_{W}$ drops below $H$ at $a_{10} \simeq 10000$. Notice that $\Gamma_{B-L}^{S}$ already becomes irrelevant at $a_{9} \simeq 8300$.

The decays and inverse decays of thermal neutrinos lead to a continuous production of a thermal asymmetry with a negative sign until the rate $\Gamma_{B-L}^{T}$ is overcome by $\Gamma_{W}$ at 
Inverse temperature $M_{1} / T$
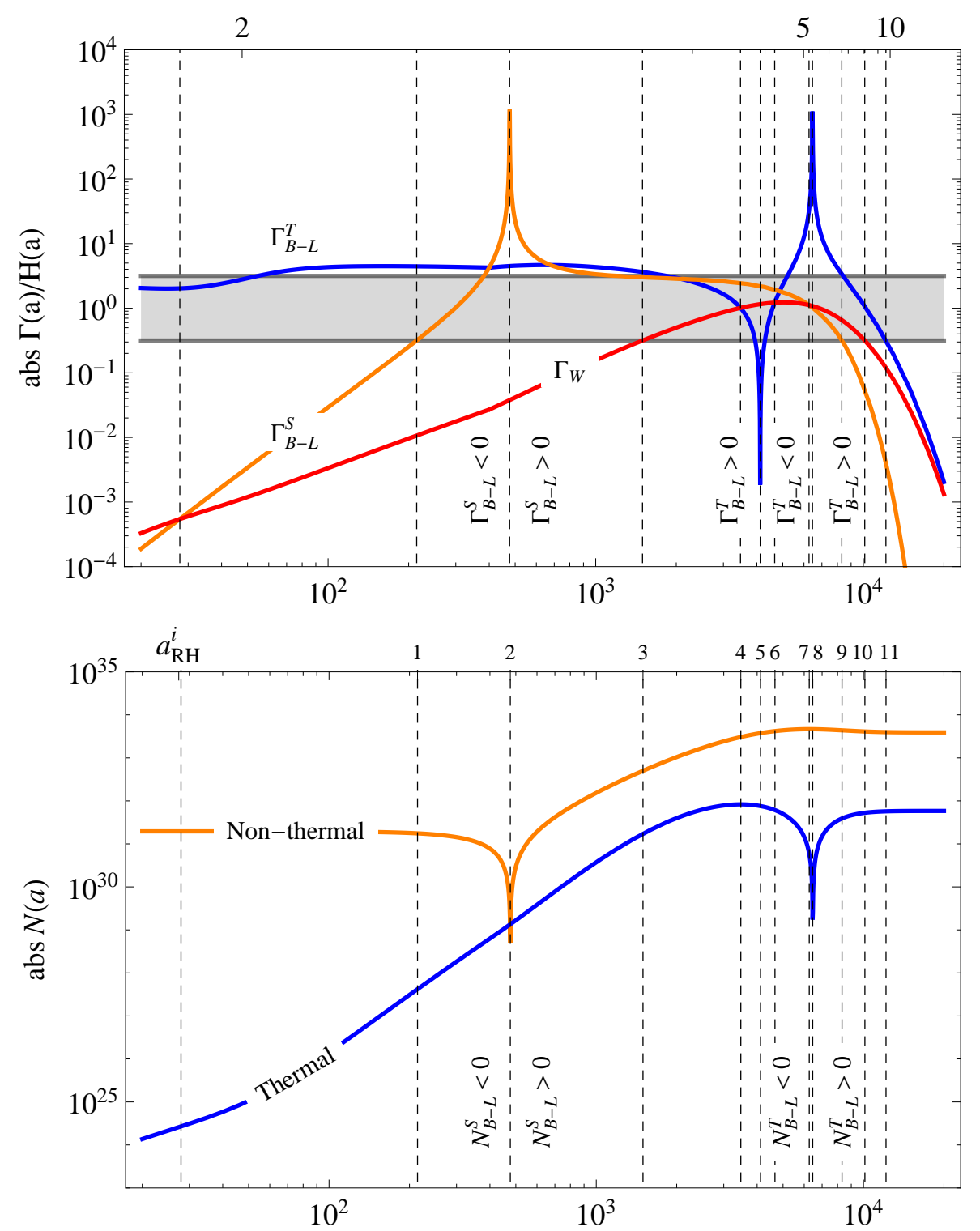

Scale factor $a$

Figure 2: Evolution with the scale factor a of (upper panel) the interaction rates $\Gamma_{B-L}^{S}, \Gamma_{B-L}^{T}$ and $\Gamma_{W}$ normalized to the Hubble rate $H$, and of (lower panel) the nonthermal and thermal parts $N_{B-L}^{S}$ and $N_{B-L}^{T}$ of the generated $B-L$ asymmetry. The rates are defined in Eq. (94), and the asymmetries were introduced in Eqs. 80a and 80b. The dashed lines and the integer numbers above the top frame edge in the lower panel refer to the various values of the scale factor and their numbering as used in the discussion of this figure in Section 4.2. The gray band in the upper panel indicates where the interaction rates are of the same order as the Hubble rate $H$. 
$a_{4} \simeq 3500$. Following that moment, washout processes push $N_{B-L}^{T}$ back to $N_{B-L}^{T}=0$. This development is reinforced by thermal neutrino decays once $N_{N_{1}}^{T}$ has exceeded the equilibrium number density $N_{N_{1}}^{\mathrm{eq}}$ at $a_{5} \simeq 4100$. Until $a_{8} \simeq 6500$ the thermal asymmetry is then converted into a positive asymmetry. After $a_{6} \simeq 4700$ the rate $\Gamma_{B-L}^{T}$ permanently dominates over $\Gamma_{W}$, and the thermal asymmetry does not decrease anymore after $a_{8}$. Instead it freezes out at its maximum value when $\Gamma_{B-L}^{T} / H \sim \mathcal{O}(0.1)$ at $a_{11} \simeq 12000$.

Finally, parallel to the onset of entropy production at $a_{R H}^{i} \simeq 27$, the rate $\Gamma_{B-L}^{S}$ exceeds $\Gamma_{W}$, and $\Gamma_{B-L}^{T}$ slightly increases.

\subsection{Radiation temperature}

Having solved the Boltzmann equation 82 for the number density of radiation quanta, we obtain from Eq. (30) the evolution of the plasma temperature $T$ which is plotted in Fig. 3. We find that the reheating process between $a_{R H}^{i} \simeq 27$ and $a_{R H}^{f} \simeq 5800$ is accompanied by an approximate temperature plateau around $T \sim 6 \times 10^{9} \mathrm{GeV}$. Especially until $S$ boson decay around $a\left(t_{S}\right) \simeq 400$ the temperature is essentially constant. This is due to the continuous production of nonthermal neutrinos which do not efficiently decay before $a\left(t_{1}\right) \simeq 1200$. With nonrelativistic $S$ bosons still representing the dominant contribution to the energy density, the comoving $N_{N_{1}}^{S}$ number density approximately scales like $N_{N_{1}}^{S} \propto \int_{t_{2}}^{t} d t^{\prime} \propto a^{3 / 2}$. According to the Boltzmann equation (82) for radiation, the comoving number density $N_{R}$ then grows like the volume, implying a constant temperature

$$
a H \frac{d}{d a} N_{R} \propto N_{N_{1}}^{S} \propto a^{3 / 2}, \quad N_{R} \propto a^{3}, \quad T=\text { const. }
$$

Once the production of nonthermal neutrinos ceases, not as much radiation is produced anymore and the temperature begins to drop. During the phases of adiabatic expansion $T$ decreases like the inverse of the scale factor, $T \propto 1 / a$.

The actual reheating temperature $T_{R H}$ is reached once the Hubble rate $H$ becomes as small as the effective decay rate $\Gamma_{N_{1}}^{S}$ of the nonthermal neutrinos (cf. Appendix C)

$$
\Gamma_{N_{1}}^{S}\left(t_{R H}\right)=H\left(t_{R H}\right), \quad T_{R H}=T\left(t_{R H}\right)
$$

For the chosen set of parameters this happens at $a\left(t_{R H}\right) \simeq 3000$, with $H=\Gamma_{N_{1}}^{S}=52 \mathrm{GeV}$, and the corresponding temperature turns out to be

$$
T_{R H} \simeq 4.1 \times 10^{9} \mathrm{GeV}
$$

A detailed discussion of how this result for the reheating temperature can be estimated on the basis of the input parameters is given in Appendix C. 

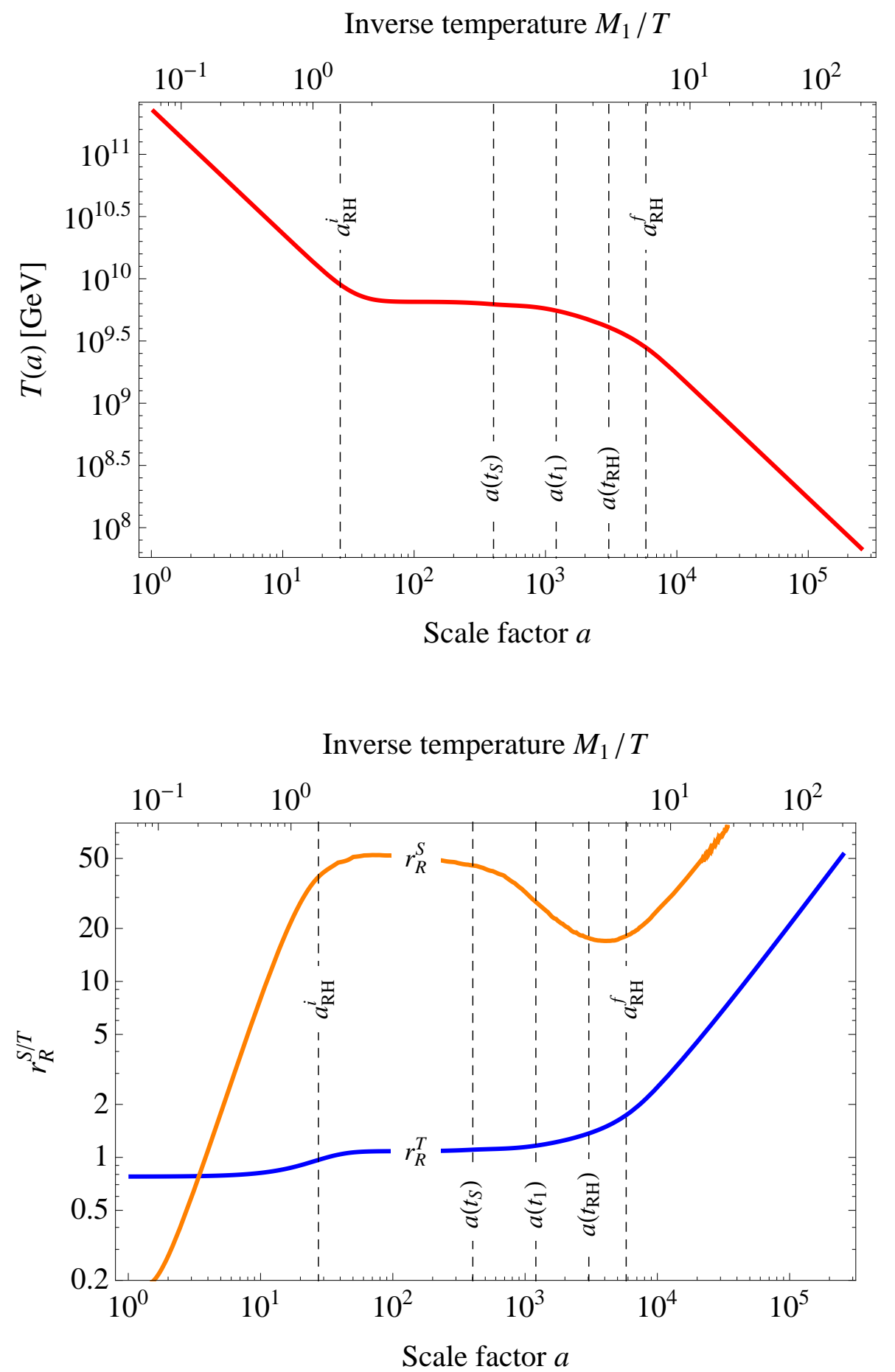

Figure 3: (Upper panel) Temperature $T$ and (Lower panel) the nonthermal and thermal correction factors $r_{R}^{S}$ and $r_{R}^{T}$ as functions of the scale factor. The temperature is calculated from Eq. (30), the correction factors were introduced in Eq. 83) and are used in the Boltzmann equation (82) for radiation to ensure a correct counting of radiation quanta. 
The lower panel in Fig. 3 presents the evolution of $r_{R}^{S}$ and $r_{R}^{T}$ as functions of the scale factor $a$, the two correction factors which effectively keep track of the average energy

per nonthermal / thermal neutrino $\varepsilon_{N_{1}}^{S / T}$ in relation to the typical radiation energy $\varepsilon_{R}$ as discussed in Sections 3.2.1 and 3.2.5. Until the onset of reheating, $r_{R}^{S}$ steeply rises. This is the consequence of an adiabatically dropping temperature and, on top of that, the progressively increasing effectiveness of the $S$ boson decays which push the average $N_{N_{1}}^{S}$ energy more and more towards $m_{S} / 2$. Between $a_{R H}^{i}$ and $a\left(t_{S}\right)$ the temperature stays rather constant and, as we have checked numerically, $\varepsilon_{N_{1}}^{S}$ has saturated close to $m_{S} / 3$. Hence, $r_{R}^{S}$ only varies little around a value of $r_{R}^{S} \simeq 50$ during that time. After $a\left(t_{S}\right)$ the $S$ boson decays become less frequent, the $N_{N_{1}}^{S}$ energies are redshifted and $r_{R}^{S}$ decreases. This trend is stopped around $a\left(t_{1}\right)$ when the temperature begins to fall again and the decay of the nonthermal neutrinos themselves sets in. These decays tend to remove rather long-lived and hence redshifted neutrinos from the spectrum leading to an increase in $\varepsilon_{N_{1}}^{S}$. Finally, after reheating the evolution of $r_{R}^{S}$ is again dominated by the adiabatically decreasing temperature.

The initial value of the thermal correction factor, $r_{R}^{T}\left(t_{2}\right) \simeq 0.78$, is close to $49 / 60$ and hence to what is expected for a relativistic fermion coupled to the massless degrees of freedom of the MSSM. The fact that it is even a bit larger is due to the negligible imprecision of calculating $\varepsilon_{N_{1}}^{T}$ by means of classical statistics (cf. Eq. (84)). Once the temperature has dropped below $M_{1}$ around $a \simeq 17$, the thermal neutrinos become nonrelativistic and $r_{R}^{T}$ increases. This evolution is only delayed by the constant temperature during reheating. After reheating $r_{R}^{T}$ continuous to increase like $r_{R}^{T} \propto a$.

\subsection{Gravitino dark matter}

The present contribution from gravitinos to the total energy density is given by

$$
\Omega_{\widetilde{G}}=\left.\frac{m_{\widetilde{G}} n_{\gamma}^{0}}{\rho_{c}} \frac{g_{*, s}^{0}}{g_{\star, s}} \frac{N_{\widetilde{G}}}{N_{\gamma}}\right|_{a_{f}},
$$

where $\rho_{c}=1.052 \times 10^{-5} h^{2} \mathrm{GeV} \mathrm{cm}{ }^{-3}$ is the critical density and $n_{\gamma}^{0}=410 \mathrm{~cm}^{-3}$ is the number density of CMB photons. Recall that after fixing all other parameters we have chosen $M_{1}$ such the gravitino abundance equals the observed one for dark matter

$$
\Omega_{\widetilde{G}} h^{2} \simeq 0.11 .
$$

In Appendix $\mathrm{D}$ we demonstrate that this result can be easily reproduced by means of certain semi-analytical estimations. 
Inverse temperature $M_{1} / T$

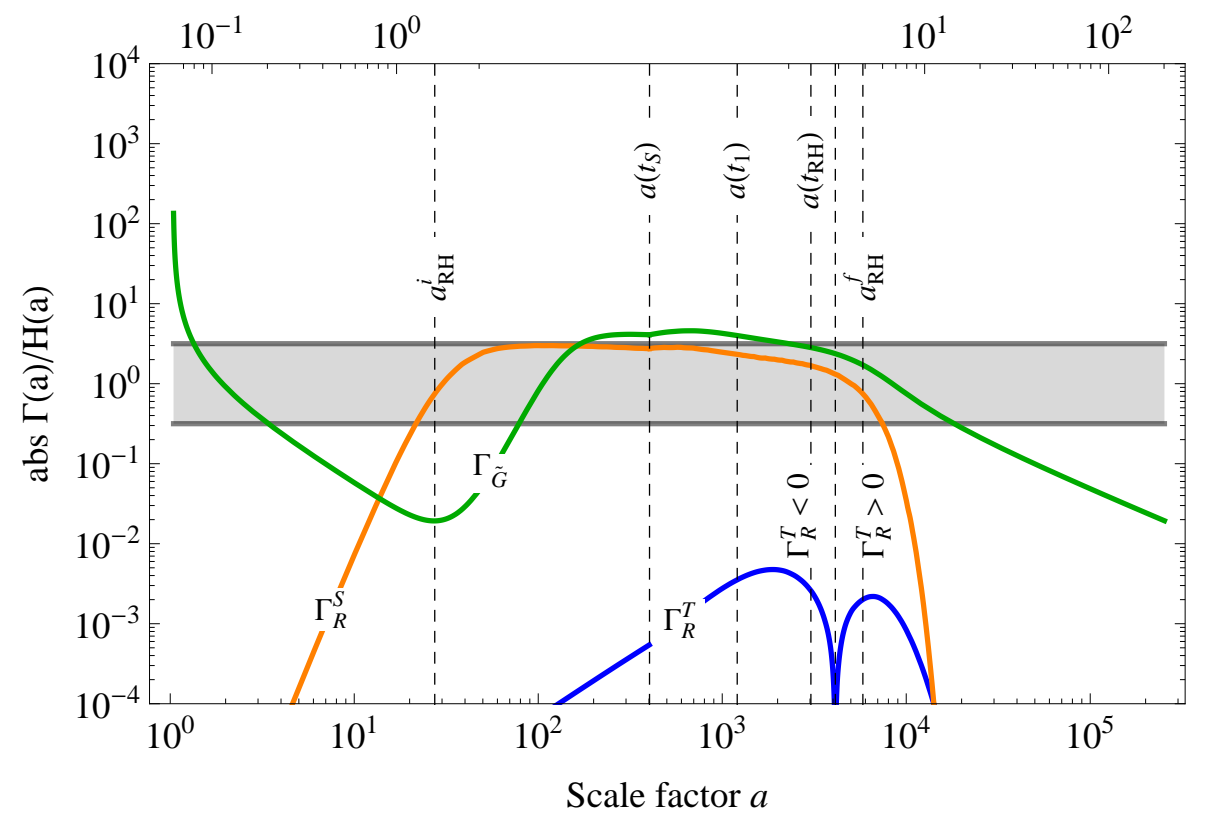

Figure 4: Interaction rates $\Gamma_{\widetilde{G}}=\gamma_{\widetilde{G}} / n_{\widetilde{G}}$, $\Gamma_{R}^{S}$ and $\Gamma_{R}^{T}$ relevant for the production of gravitinos and radiation, normalized to the Hubble rate $H$. The rates $\Gamma_{R}^{S}$ and $\Gamma_{R}^{T}$ were introduced in Eq. (100). The gray band indicates where the interaction rates are of the same order as the Hubble rate $H$.

The evolution of the gravitino abundance with time is controlled by the production rate $\Gamma_{\widetilde{G}}=\gamma_{\widetilde{G}} / n_{\widetilde{G}}$ (cf. Eqs. (85) and (86)), which strongly depends on the temperature. To demonstrate the close relation between the production of gravitinos and that of radiation we plot $\Gamma_{\widetilde{G}}$ in Fig. 4 together with the rates $\Gamma_{R}^{S}$ and $\Gamma_{R}^{T}$ at which radiation is produced due to nonthermal and thermal neutrino decay

$$
\Gamma_{R}^{S}=r_{R}^{S} \frac{N_{N_{1}}^{S}}{N_{R}} \Gamma_{N_{1}}^{S}, \quad \Gamma_{R}^{T}=r_{R}^{T} \frac{N_{N_{1}}^{T}-N_{N_{1}}^{\mathrm{eq}}}{N_{R}} \Gamma_{N_{1}}^{T} .
$$

As expected we find that only the decay of the nonthermal neutrinos efficiently influences the radiation abundance. Between $a \simeq 22$ and $a \simeq 7400$, which is basically identical to the time interval in which reheating takes place, $\Gamma_{R}^{S}$ is of the same order as the Hubble rate. $\Gamma_{R}^{S} / H$ reaches its maximal value before $S$ boson decay around $a\left(t_{S}\right)$, subsequently decreases a bit and finally drops off shortly after $a_{R H}^{f}$. The ratio $\Gamma_{R}^{T} / H$ is at most of order $\mathcal{O}\left(10^{-2}\right)$ which is the case towards the end of reheating when the thermal neutrinos are close to thermal equilibrium. The rate $\Gamma_{\widetilde{G}}$ traces the efficiency of the nonthermal neutrino decays: While the $N_{1}^{S}$ decays are not active yet, $\Gamma_{\widetilde{G}} / H$ decreases due to the falling temperature. But as soon as $\Gamma_{R}^{S}$ becomes competitive with the Hubble rate around 
$a_{R H}^{i}, \Gamma_{\widetilde{G}} / H$ bends over and eventually it reaches values of order $\mathcal{O}(1)$ and even larger. On the other hand, once the nonthermal neutrino decay has ended, $\Gamma_{\widetilde{G}}$ returns to its ordinary behaviour that we expect for adiabatic expansion. In total gravitino production occurs between $a \simeq 79$ and $a \simeq 18000$. The main part of the gravitino abundance is, hence, produced towards the end or after reheating.

\section{Results and discussion}

The parameter point selected in the previous section was chosen such that we readily obtained the right baryon asymmetry and gravitino abundance. Now we extend our discussion to a quantitative analysis of the entire parameter space and determine the bounds within which consistency between successful leptogenesis and gravitino dark matter can be reached. According to the flavour model introduced in Section 2 we are free to vary the neutrino mass parameters $M_{1}, \widetilde{m}_{1}$ and $v_{B-L}$. On the supergravity side the gravitino and gluino masses $m_{\widetilde{G}}$ and $m_{\tilde{g}}$ represent free parameters (cf. Section 3.2.6). Moving in parameter space changes the interaction rates relevant to our scenario, most notably the production and decay rates of the $N_{1}$ neutrino. This has consequences for the reheating process (cf. Section 5.1), the generation of the baryon asymmetry (cf. Section 5.2) and the thermal production of gravitinos (cf. Section 5.3). By imposing the two conditions [20]

$$
\begin{gathered}
\eta_{B} \equiv \eta_{B}^{S}+\eta_{B}^{T} \geq \eta_{B}^{\text {obs }} \simeq 6.2 \times 10^{-10}, \\
\Omega_{\widetilde{G}} h^{2}=\Omega_{\mathrm{DM}} h^{2} \simeq 0.11,
\end{gathered}
$$

we are able to identify the regions in parameter space in which both, the present baryonto-photon ratio and the dark matter density are successfully generated. In this manner, we obtain a link between neutrino and superparticle masses. The parameter dependence of the reheating temperature and the interplay of nonthermal and thermal leptogenesis follow along the way.

From the allowed range for the $B-L$ breaking scale (cf. Eq. (10)), we consider the boundary values and an intermediate scale. All three values are associated with different ranges for the heavy Majorana mass $M_{1}$ (cf. Eq. (7a)),

$$
\begin{array}{ll}
v_{B-L}=3.4 \times 10^{12} \mathrm{GeV}: & 1.3 \times 10^{5} \mathrm{GeV} \leq M_{1} \leq 1.1 \times 10^{10} \mathrm{GeV}, \\
v_{B-L}=5.8 \times 10^{13} \mathrm{GeV}: & 2.1 \times 10^{6} \mathrm{GeV} \leq M_{1} \leq 1.9 \times 10^{11} \mathrm{GeV}, \\
v_{B-L}=1.0 \times 10^{15} \mathrm{GeV}: & 3.7 \times 10^{7} \mathrm{GeV} \leq M_{1} \leq 3.3 \times 10^{12} \mathrm{GeV} .
\end{array}
$$


Furthermore, in order to take into account the $\mathcal{O}(1)$ uncertainties in the Yukawa couplings $h^{\nu}$, we allow the effective neutrino mass $\widetilde{m}_{1}$ to vary in the range

$$
10^{-5} \mathrm{eV} \leq \widetilde{m}_{1} \leq 10^{-1} \mathrm{eV}
$$

The gravitino mass is taken from the interval

$$
30 \mathrm{MeV} \leq m_{\widetilde{G}} \leq 700 \mathrm{GeV} .
$$

In view of the present bound on the gluino mass, $m_{\tilde{g}} \gtrsim 700 \mathrm{GeV}$, imposed by collider searches [35,36], we use a mass of $m_{\tilde{g}}=800 \mathrm{GeV}$ as a representative value in this section. Different choices of $m_{\tilde{g}}$ would lead to similar qualitative results, the only difference being that all values of $m_{\widetilde{G}}$ would have to be rescaled (cf. Section 5.3 and Appendix D).

In all plots of the parameter space presented in this section as well as in the Appendices $\mathrm{C}$ and $\mathrm{D}$ (Figs. 5, 6, 7, 8, 10, 13 and 14) we mark the positions of the two parameter points listed in Tab. 2, The point the discussion in Section 4 was based on as well as the point that was investigated in Ref. [3].

\section{$5.1 \quad$ Reheating temperature}

The concept of temperature is only applicable as long as the interactions in the system under study are in thermal or, at least, kinetic equilibrium. Hence, regarding our scenario, it is not before the creation of an initial thermal bath due to quick thermalization of the $N_{2,3}$ decay products that we can meaningfully speak about a temperature. Subsequently, the main part of the energy density continues to reside in nonthermal particles. At first most of the energy density is carried by the $S$ bosons, and then, from $t \simeq t_{S}$ onwards, by the nonthermal $N_{1}$ neutrinos. The energy transfer to the thermal bath, i.e. the reheating of the universe, becomes fully efficient when the $N_{1}$ neutrinos decay into standard model radiation. In first approximation, this happens once the Hubble rate $H$ has dropped to the value of the effective decay rate $\Gamma_{N_{1}}^{S}$ of the nonthermal $N_{1}$ neutrinos,

$$
\Gamma_{N_{1}}^{S}\left(t_{R H}\right)=H\left(t_{R H}\right), \quad T_{R H}=T\left(t_{R H}\right),
$$

where $\Gamma_{N_{1}}^{S}$ is the vacuum decay rate $\Gamma_{N_{1}}^{0}$ weighted with the inverse time dilatation factor as defined in Eq. 666). The reheating temperature $T_{R H}$ can then be obtained by applying Eq. (105) to the solutions of the Boltzmann equations. ${ }^{6}$

\footnotetext{
${ }^{6}$ As discussed in Section 3 , we use an approximate solution of the Friedmann equation, $H=\dot{a} / a$, with the scale factor $a(t)$ given in Eq. (36).
} 

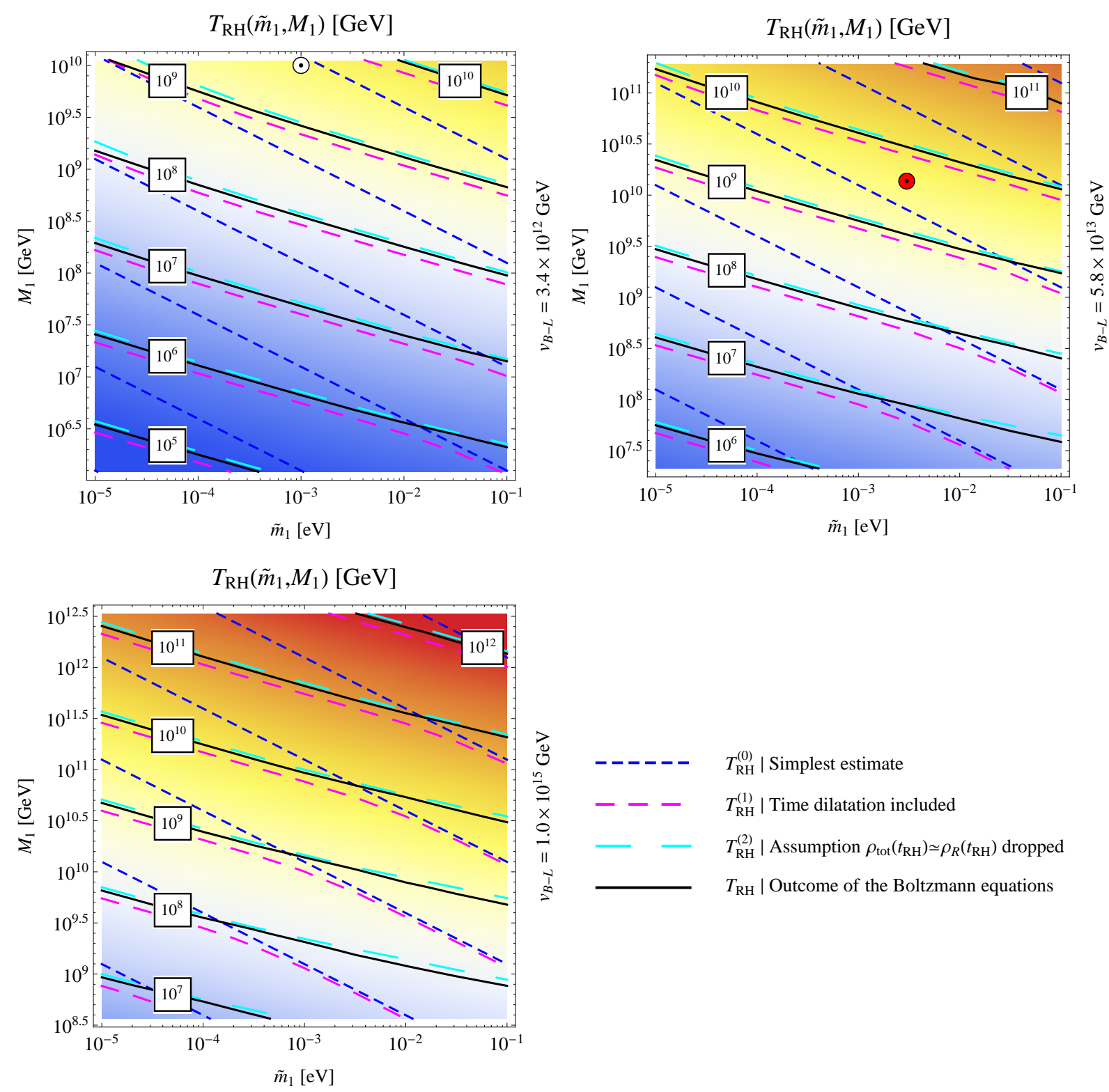

Figure 5: Contour plots of the reheating temperature $T_{R H}$ as a function of the parameters $\widetilde{m}_{1}$ and $M_{1}$ for the three different choices of $v_{B-L}$ listed in Eq. 102). The outcome of the Boltzmann equations $T_{R H}$, calculated according to Eq. 105, is compared with three different estimates $T_{R H}^{(0)}, T_{R H}^{(1)}$ and $T_{R H}^{(2)}$ which are respectively defined in Eqs. (106), (C.8) and (C.9). The contour labels as well as the background colours indicate the numerical values of $T_{R H}$. Going to smaller values of $\widetilde{m}_{1}$ the $T_{R H}^{(0)}$ and $T_{R H}^{(1)}$ contours approach the corresponding $T_{R H}$ contours from below. With respect to the $T_{R H}$ contours the $T_{R H}^{(2)}$ contours are shifted upwards by approximately $\Delta \log _{10} T_{R H} \simeq 0.04$, cf. Eq. C.11. 
For nonrelativistic Majorana neutrinos $N_{1}^{S}$, instantaneous energy transfer and $H$ being determined from the Friedmann equation, the reheating temperature is given by

$$
T_{R H}^{(0)}=\left(\frac{90}{8 \pi^{3} g_{\star, \rho}}\right)^{1 / 4} \sqrt{\Gamma_{N_{1}}^{0} M_{p}} .
$$

In our scenario we do not meet either of these conditions which is why the simple estimate $T_{R H}^{(0)}$ has to be augmented with several corrections in order to properly reproduce the outcome of the Boltzmann equations:

- Being produced in $S$ decays, the nonthermal neutrinos all carry initial energy $m_{S} / 2 \simeq 150 M_{1}$. For most of the time they are, hence, highly relativistic such that their decays occur at an effective rate $\Gamma_{N_{1}}^{S}$ (cf. Eq. (105)). Replacing $\Gamma_{N_{1}}^{0}$ by $\Gamma_{N_{1}}^{S}$ results in an approximation $T_{R H}^{(1)}$ for the reheating temperature;

- At the time when $T_{R H}$ is evaluated a large fraction of the energy density still resides in nonthermal neutrinos. Taking into account that only a part of the total energy density at $t=t_{R H}$ contributes to $T_{R H}$ yields an approximation $T_{R H}^{(2)}$;

- The fact that our approximation for the Hubble rate $H=\dot{a} / a$ does not fulfill the Friedmann equation exactly introduces a final imprecision which effectuates the remaining small deviation of $T_{R H}^{(2)}$ from the actual reheating temperature $T_{R H}$.

We refer the interested reader to Appendix C where the reconstruction of the numerical result $T_{R H}$ starting from the simplest estimate $T_{R H}^{(0)}$ is discussed in greater detail.

The reheating temperature $T_{R H}$ obtained from the Boltzmann equations for the three values of $v_{B-L}$ in Eq. (102) is presented in Fig. 5 together with the different approximations $T_{R H}^{(i)}$ as a function of the neutrino mass parameters $\widetilde{m}_{1}$ and $M_{1}$. Notice that the behaviour of $T_{R H}^{(0)}$ is determined by the width $\Gamma_{N_{1}}^{0} \propto \widetilde{m}_{1} M_{1}^{2}$ (cf. Eq. 12), which is independent of $v_{B-L}$. The correction due to the time dilatation factor mainly depends on the ratio of the Majorana neutrino decay width Eq. 12 ) and the $S$ boson decay width Eq. (22),

$$
\frac{\Gamma_{N_{1}}^{0}}{\Gamma_{S}^{0}} \propto \frac{\widetilde{m}_{1} v_{B-L}^{2}}{M_{1} v_{E W}^{2}} .
$$

For $\Gamma_{N_{1}}^{0} \gg \Gamma_{S}^{0}$, the bulk of the nonthermal neutrinos decaying at $t=t_{R H}$ is produced just shortly before and is therefore relativistic. On the other hand, for $\Gamma_{N_{1}}^{0} \ll \Gamma_{S}^{0}$, most of the nonthermal neutrinos decaying at the reheating time are nonrelativistic. For fixed $v_{B-L}$, this correction turns out to be marginal for the smallest effective neutrino masses $\widetilde{m}_{1}$ and the largest Majorana neutrino masses $M_{1}$. The correction increases with the ratio 
in Eq. (107) becoming larger. Its maximum is given by the flavour model, $\sqrt{2 M_{1} / m_{S}} \simeq$ $\sqrt{1 / 150}$. The related correction corresponding to the overestimation of the energy density of radiation at $t=t_{R H}$ has the same dependence on parameters. Finally, the mismatch between the Hubble rate and the exact solution of the Friedmann equation only slightly modifies the reheating temperature. All in all, the global effect of these corrections is to increase (decrease) the dependence of the reheating temperature on $M_{1}\left(\widetilde{m}_{1}\right)$.

In each of the three panels of Fig.5, corresponding to the three different values of $v_{B-L}$, the values of $\widetilde{m}_{1}$ and $M_{1}$ respectively span four orders of magnitude allowing for reheating temperatures ranging over five orders of magnitude. Reheating temperatures as small as $T_{R H} \simeq 10^{5} \mathrm{GeV}$ are obtained for the lowest decay rates in association with the smallest initial false vacuum energy density, i.e. for the minimal values of $v_{B-L}, \widetilde{m}_{1}$ and $M_{1}$. Conversely, reheating temperatures as large as $T_{R H} \simeq 10^{12} \mathrm{GeV}$ are obtained for the maximal values of $v_{B-L}, \widetilde{m}_{1}$ and $M_{1}$.

Lastly, we observe that the region where the reheating temperature exceeds the Majorana neutrino mass significantly shrinks when going from the simplest approximation $T_{R H}^{(0)}$ to the results of the Boltzmann equations $T_{R H}$. As for the former, $T_{R H}^{(0)}>M_{1}$ for $\widetilde{m}_{1} \gtrsim 2 \times 10^{-3} \mathrm{eV}$, independent of $M_{1}$, while in the latter case $T_{R H}>M_{1}$ is only accomplished for the largest values of $\widetilde{m}_{1}$ and $M_{1}$. The reasons for this relative decrease in the reheating temperature were already mentioned following Eq. (106): the longer neutrino lifetimes due to their relativistic nature and the overestimation of radiation energy in deriving $T_{R H}^{(0)}$. As the strength of the washout processes during the generation of the baryon asymmetry crucially depends on the ratio of temperature $T$ and neutrino mass $M_{1}$ we expect the efficiency of leptogenesis to severely drop in the region $T_{R H}>M_{1}$.

\subsection{Baryon asymmetry}

The baryon asymmetry that is generated for a given choice of input parameters follows from the respective solutions of the Boltzmann equations according to Eq. (91). In this section we shall discuss in turn the contributions it receives from the decays of the nonthermal and thermal neutrinos. Our main results are displayed in Fig.6 which presents the baryon asymmetry for the three values of the $B-L$ breaking scale (cf. Eq. 102) as function of the neutrino mass parameters $\widetilde{m}_{1}$ and $M_{1}$. In each panel of Fig. 66, we indicate the regions in which leptogenesis from the decay of nonthermal (light green) and thermal neutrinos (gray-green) successfully produces the observed baryon asymmetry. Notice that in some regions of parameter space (dark green) both variants of leptogenesis manage to overcome the observational bound individually while in others (white) $\eta_{B}^{\text {obs }}$ only is 
exceeded after taking the sum of the two contributions.

The blue solid lines in Fig.6 separate the parameter regions in which leptogenesis is respectively driven either by the decay of the nonthermal or the thermal neutrinos. In the viable regions of parameter space the nonthermal contribution to the baryon asymmetry typically represents the clearly dominant one. As we have checked numerically, the generated asymmetry in that case can be reconstructed to good approximation by assuming that at $t=t_{1}$ the energy density of the nonthermal neutrinos is almost instantaneously converted into radiation. Nonthermal neutrinos of average energy $\varepsilon_{N_{1}}^{S}$ (cf. Eq. (84)), that rapidly decay around $t=t_{1}$, lead to an asymmetry (cf. [37])

$$
\left.\eta_{B}^{\mathrm{rapid}} \simeq 7 \frac{3}{4} c_{\mathrm{sph}} \epsilon_{1} \frac{T}{\varepsilon_{N_{1}}^{S}}\right|_{t=t_{1}}
$$

We emphasize that neglecting the relativistic motion of the nonthermal neutrinos, i.e. employing simply the mass $M_{1}$ instead of the full energy per particle $\varepsilon_{N_{1}}^{S}$ in Eq. (108), would entail an asymmetry proportional to the temperature, $\eta_{B}^{\text {rapid }} \propto T\left(t_{1}\right)$. Such an estimate fails to reproduce our results except for some accidental points in parameter space.

For fixed $v_{B-L}$ and $\widetilde{m}_{1} \lesssim \mathcal{O}\left(10^{-3}\right) \mathrm{eV}$ the nonthermal baryon asymmetry does not depend on $\widetilde{m}_{1}$ anymore. This observation can be easily understood in terms of the Boltzmann equation (80a) for $N_{B-L}^{S}$. For very small effective neutrino masses, the washout processes become inefficient, leaving us only with the production term. The size of the final asymmetry then only depends on the maximal $N_{1}^{S}$ abundance that can be reached in the course of $S$ boson decay, which is reminiscent of standard thermal leptogenesis in the weak washout regime. Since the collision operator accounting for the production of nonthermal neutrinos through the decays of $S$ bosons as well as the CP parameter $\epsilon_{1}$ are solely controlled by the Majorana neutrino mass, the resulting baryon asymmetry ends up being exclusively determined by $M_{1}$.

Increasing $v_{B-L}$ for fixed neutrino masses $\widetilde{m}_{1}$ and $M_{1}$ reduces the produced baryon asymmetry. This is due to several effects whose influence is apparent in Eq. (108): On the one hand a higher $B-L$ breaking scale implies a larger relativistic correction resulting in a smaller effective decay rate $\Gamma_{N_{1}}^{S}$, on the other hand it leads to a faster Hubble expansion. The former increases $\varepsilon_{N_{1}}^{S} \sim\left\langle M_{1} / E_{N_{1}}\right\rangle_{S}^{-1} M_{1}$ and delays the neutrino decays such that Eq. (108) needs to be evaluated at a later time $t_{1}$ corresponding to a smaller temperature $T$. The faster Hubble rate $H$ reinforces the drop-off in the temperature. We

\footnotetext{
${ }^{7}$ This actually happens in Ref. [3] in which $\eta_{B}^{\text {rapid }}$ is calculated for the corresponding set of parameter values given in Tab. 2 ,
} 

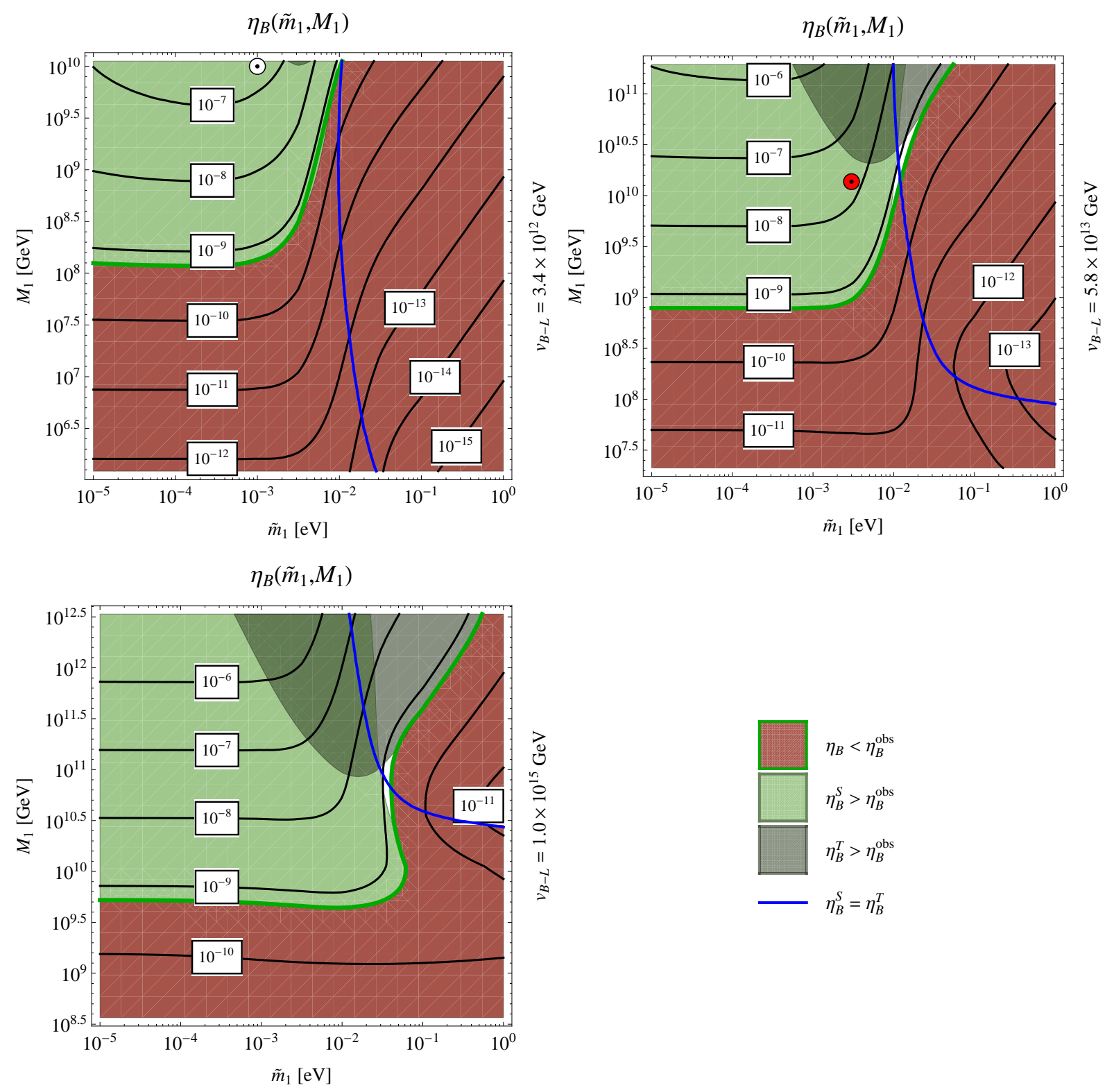

Figure 6: Contour plots of the baryon-to-photon ratio $\eta_{B}$ as defined in Eq. (91) as a function of the parameters $\widetilde{m}_{1}$ and $M_{1}$ for the three different choices of $v_{B-L}$ listed in Eq. 102). In the light green (graygreen) region leptogenesis through the decay of nonthermal (thermal) Majorana neutrinos successfully reproduces the observed baryon asymmetry $\eta_{B}^{o b s}$. In the red region the total asymmetry is not able to overcome the observational bound. The blue line separates the regions in which each leptogenesis variant is the dominant one. 
may reformulate this argument in terms of the rates $\Gamma_{N_{1}}^{S}$ and $H$ by saying that a smaller ratio $\Gamma_{N_{1}}^{S} / H$ reflects a lower efficiency of the nonthermal neutrino decays. From this point of view, the generation of the asymmetry struggles to keep pace with the expansion of the universe resulting in a more diluted asymmetry.

At values of $\widetilde{m}_{1}$ larger than $\mathcal{O}\left(10^{-3}\right) \mathrm{eV}$ resonant $\ell H \leftrightarrow \bar{\ell} \bar{H}$ scatterings that wash out the generated asymmetry at a rate $\Gamma_{W}$ (cf. Eq. (94)) dramatically decrease the efficiency of nonthermal leptogenesis: For small $\widetilde{m}_{1}$ we have $T_{R H} \ll M_{1}$ (cf. Fig. 5) and the production of on-shell $N_{1}$ neutrinos out of the thermal bath is Boltzmann suppressed,

$$
T \ll M_{1}: \quad \Gamma_{W}=\frac{N_{N_{1}}^{\mathrm{eq}}}{2 N_{\ell}^{\mathrm{eq}}} \Gamma_{N_{1}}^{T} \propto\left(\frac{M_{1}}{T}\right)^{3 / 2} e^{-M_{1} / T} \Gamma_{N_{1}}^{0} \ll \Gamma_{N_{1}}^{0} .
$$

But as $\widetilde{m}_{1}$ becomes larger, the reheating temperature approaches $M_{1}$ and the final asymmetry is depreciated due to washout. For given $\widetilde{m}_{1}$ and $M_{1}$, increasing $v_{B-L}$ results in a decrease of the reheating temperature (cf. Section 5.1). In particular, this reduces the region in parameter space where $T_{R H}>M_{1}$, consequently extending the region in which nonthermal leptogenesis can successfully proceed without being much affected by washout.

The decay of the nonthermal neutrinos is not the only mechanism by means of which the baryon asymmetry is generated in our scenario. It also receives a contribution $\eta_{B}^{T}$ from the decays of the thermally produced neutrinos $N_{1}^{T}$. If $M_{1}$ is sufficiently large, $\eta_{B}^{T}$ can exceed the observed baryon asymmetry on its own. Its behaviour in parameter space is similar to the one of standard thermal leptogenesis with vanishing initial neutrino abundance. However, it is important to note that standard thermal leptogenesis differs from our thermal mechanism in the sense that in the former the thermal bath out of which the Majorana neutrinos are produced is assumed to have an independent origin (e.g., inflaton decay) and the initial temperature usually is taken to be arbitrarily high. By contrast, the generation of our thermal asymmetry $\eta_{B}^{T}$ is tightly coupled to the dynamics of reheating in the course of the nonthermal neutrino decays. In standard thermal leptogenesis the CP asymmetry $\epsilon_{1}$ (cf. Eq. (14)) as well as the evolution of the $N_{1}$ and $B-L$ abundances are controlled by the neutrino mass parameters $\widetilde{m}_{1}$ and $M_{1}$. To guarantee successful leptogenesis, $M_{1}$ is constrained to be at least $\mathcal{O}\left(10^{9}\right) \mathrm{GeV}$ if $\widetilde{m}_{1}$ is fixed at $\widetilde{m}_{1} \simeq 10^{-3} \mathrm{GeV}$. Effective neutrino masses $\widetilde{m}_{1}$ different from that result in larger bounds on $M_{1}$.

In our scenario, the values of $M_{1}$ above which thermal leptogenesis is efficient are comparatively one to two orders of magnitude larger. Tab. 3 summarizes the respective bounds on $M_{1}$ for the three different $B-L$ breaking scales together with the corresponding values of $\widetilde{m}_{1}$ and $T_{R H}$. The fact that now $M_{1}$ has to be much larger than $\mathcal{O}\left(10^{9}\right) \mathrm{GeV}$ 


\begin{tabular}{cccccc} 
Panel & $v_{B-L}[\mathrm{GeV}]$ & $\widetilde{m}_{1}[\mathrm{eV}]$ & $M_{1}[\mathrm{GeV}]$ & $T_{R H}[\mathrm{GeV}]$ & $T_{R H} / M_{1}$ \\
\hline \hline 1 & $3.4 \times 10^{12}$ & $3.2 \times 10^{-3}$ & $1.0 \times 10^{10}$ & $6.4 \times 10^{9}$ & 0.63 \\
2 & $5.8 \times 10^{13}$ & $5.2 \times 10^{-3}$ & $2.1 \times 10^{10}$ & $7.9 \times 10^{9}$ & 0.38 \\
3 & $1.0 \times 10^{15}$ & $1.6 \times 10^{-2}$ & $8.5 \times 10^{10}$ & $2.0 \times 10^{10}$ & 0.23 \\
\hline \hline
\end{tabular}

Table 3: Parameter points in the three panels of Fig. 6 corresponding to the lowest possible values of $M_{1}$ for which the decay of the thermal neutrinos suffices to reproduce the observed baryon asymmetry. The values for the reheating temperature follow from Fig. 5 .

finds its origin in the interplay between the specific reheating process at work and the temperature dependence of thermal leptogenesis: First of all, in the discussion of the decay of the nonthermal neutrinos we saw that the temperature is bounded from above to prevent complete washout of the asymmetry. The same holds for thermal leptogenesis; but in this case the temperature also must not be too low in order to ensure an efficient neutrino production from the thermal bath. Consequently, as a compromise between very small $\left(T \ll M_{1}\right)$ and very large $\left(T \gg M_{1}\right)$ temperatures, thermal leptogenesis is most efficient at $T \sim M_{1}$ (cf. Tab. 3). 8 Second, as for standard thermal leptogenesis, our thermal mechanism prefers an intermediate value of $\widetilde{m}_{1}$. Taking $\widetilde{m}_{1}$ to large values increases the strength of the washout processes. Small $\widetilde{m}_{1}$ results in a low temperature and a small neutrino decay rate $\Gamma_{N_{1}}^{0}$ such that the $N_{1}^{T}$ production becomes suppressed. When asking for the lower bounds on $M_{1}$ we thus have to look for the smallest values of $M_{1}$ for which the condition $T \sim M_{1}$ holds and $\widetilde{m}_{1}$ is neither too large nor too small. In contrast to standard thermal leptogenesis, in our scenario the accessible temperatures now also depend on the mass parameter $M_{1}$. As can be seen from Fig.5, the considered reheating process simply does not manage to satisfy the condition $T \sim M_{1}$ for $M_{1} \sim 10^{9} \mathrm{GeV}$ without entering the strong washout regime. Instead, $M_{1}$ has at least to be as large as indicated in Tab. 3 to avoid too large values of $\widetilde{m}_{1}$ while still fulfilling $T \sim M_{1}$.

Comparing the three points in Tab. 3 we note that the ratio of the reheating temperature $T_{R H}$ to $M_{1}$ decreases as $v_{B-L}$ becomes larger. The production of thermal neutrinos is, consequently, less efficient for high $v_{B-L}$. This is, however, compensated for by the increase in the CP asymmetry parameter $\epsilon_{1}$ for heavier $N_{1}$ neutrinos (cf. Eq. (15)). Likewise, the corresponding effective neutrino masses $\widetilde{m}_{1}$ increase when going to larger $B-L$

\footnotetext{
${ }^{8}$ Note that our scenario also differs from standard thermal leptogenesis because we only consider decays and inverse decays. We have checked that including $\Delta L=1$ and $\Delta L=2$ scatterings would enforce the production of Majorana neutrinos for $\widetilde{m}_{1} \lesssim 3 \times 10^{-3} \mathrm{eV}$ as it is the case in standard thermal leptogenesis [33, resulting in a slight expansion of the allowed region in the weak washout regime.
} 
breaking scales. This effect is based on the fact that for fixed $\widetilde{m}_{1}$ and $M_{1}$ an increase in $v_{B-L}$ entails a drop in the temperature. The factor representing the Boltzmann suppression in the washout rate $\Gamma_{W}$ (cf. Eq. (109) ) then becomes smaller which enables one to raise the neutrino decay width $\Gamma_{N_{1}}^{0}$ by increasing $\widetilde{m}_{1}$.

Standard thermal leptogenesis predicts a final baryon asymmetry of

$$
\eta_{B}^{\mathrm{th}}=\frac{3}{4} \frac{g_{\star}^{0}}{g_{\star}} c_{\mathrm{sph}} \epsilon_{1} \kappa_{f}\left(\widetilde{m}_{1}\right)
$$

where the final efficiency factor $\kappa_{f}$ only depends on $\widetilde{m}_{1}$. For $\widetilde{m}_{1} \gtrsim 10^{-3} \mathrm{eV}$ it may be parametrized as 33

$$
\kappa_{f}\left(\widetilde{m}_{1}\right)=2 \times 10^{-2}\left(\frac{10^{-2} \mathrm{eV}}{\widetilde{m}_{1}}\right)^{1.1} .
$$

Combining Eqs. (110) and (111) with Eq. (15), one finds that $\eta_{B}^{\text {th }}$ evolves as $\eta_{B}^{\text {th }} \propto \widetilde{m}_{1}^{-1} M_{1}$. This is exactly the behaviour of the total baryon asymmetry one observes in the regions where the thermal contribution dominates over the nonthermal one, i.e. the regions on the right-hand side of the blue lines in Fig.6. As the number density of nonthermal neutrinos usually exceeds the number density of thermal neutrinos at the time the asymmetry is created, the relative size of the two asymmetries $\eta_{B}^{S}$ and $\eta_{B}^{T}$ is controlled by the efficiency of the nonthermal mechanism. Only when the nonthermal asymmetry is suppressed due to efficient washout, the baryon asymmetry due to the decay of the thermal neutrinos has a chance to dominate.

In conclusion, it is remarkable that leptogenesis through the decay of the nonthermal Majorana neutrinos is able to widely extend the region in parameter space in which the observed baryon asymmetry can successfully be reproduced. For the lowest $B-L$ breaking

scale $v_{B-L}=3.4 \times 10^{12} \mathrm{GeV}$, Majorana neutrinos as light as $M_{1} \simeq 10^{8} \mathrm{GeV}$ are sufficient to generate the observed baryon asymmetry.

\subsection{Gravitino dark matter}

Having discussed leptogenesis on its own in the last section, we now ask for the regions in parameter space where both conditions of Eq. (101) are satisfied, i.e. in which we obtain gravitino dark matter along with a sufficient baryon asymmetry. As outlined in Section 3.2.6 the thermal production of gravitinos is controlled by three parameters: the gravitino and gluino masses $m_{\widetilde{G}}$ and $m_{\tilde{g}}$ as well as the temperature $T$. The latter is determined by the reheating process, $T_{R H}=T_{R H}\left(v_{B-L}, \widetilde{m}_{1}, M_{1}\right)$, such that $\Omega_{\widetilde{G}} h^{2}$, the present contribution from gravitinos to the energy density of the universe, depends on 
all free parameters of our scenario. For each point in parameter space the respective solutions of the Boltzmann equations allow us to calculate $\Omega_{\widetilde{G}} h^{2}$ according to Eq. (98). By imposing the condition that gravitinos be the constituents of dark matter we can then derive relations between neutrino and superparticle masses. For instance, if we fix the gluino mass at $800 \mathrm{GeV}$,

$$
\left.\Omega_{\widetilde{G}} h^{2}\left(v_{B-L}, M_{1}, \widetilde{m}_{1}, m_{\widetilde{G}}, m_{\tilde{g}}\right)\right|_{m_{\tilde{g}}=800 \mathrm{GeV}}=\Omega_{\mathrm{DM}} h^{2}
$$

we can solve for $M_{1}$ as a function of $v_{B-L}, \widetilde{m}_{1}$ and $m_{\widetilde{G}}$,

$$
M_{1}=M_{1}\left(v_{B-L}, \widetilde{m}_{1}, m_{\widetilde{G}}\right)
$$

We consider those choices of the parameters $v_{B-L}, \widetilde{m}_{1}$ and $m_{\widetilde{G}}$ as viable, which actualize gravitino dark matter for $M_{1}$ values that are accessible in the context of the flavour model (cf. Eq. 7a) ),

$$
M_{1}\left(v_{B-L}, \widetilde{m}_{1}, m_{\widetilde{G}}\right) \leq \eta^{2} v_{B-L}
$$

Furthermore, applying Eq. 113 to the results of Section 5.1 allows one to trade the $M_{1}$ dependence of the reheating temperature $T_{R H}$ for a dependence on $m_{\widetilde{G}}$,

$$
T_{R H}=T_{R H}\left(v_{B-L}, M_{1}, \widetilde{m}_{1}\right) \rightarrow T_{R H}\left(v_{B-L}, \widetilde{m}_{1}, m_{\widetilde{G}}\right)
$$

Similarly, Eq. (113) can be used to translate the bounds on $\widetilde{m}_{1}$ and $M_{1}$ shown in Fig. 6 that were obtained by requiring successful leptogenesis into bounds on $\widetilde{m}_{1}$ and $m_{\widetilde{G}}$,

$$
\eta_{B}\left(v_{B-L}, M_{1}, \widetilde{m}_{1}\right) \rightarrow \eta_{B}\left(v_{B-L}, \widetilde{m}_{1}, m_{\widetilde{G}}\right) \geq \eta_{B}^{\mathrm{obs}}
$$

The parameter points we are after, i.e. the points at which the baryon asymmetry is accounted for by leptogenesis and gravitinos constitute the dark matter, now correspond to those values of $v_{B-L}, \widetilde{m}_{1}$ and $m_{\widetilde{G}}$ that satisfy the two conditions in Eqs. (114) and (116) simultaneously. On the basis of our numerical study of the Boltzmann equations we are able to identify the regions of interest in parameter space: Fig.7 presents our results in combination with the associated values of $M_{1}$ (cf. Eq. (113)), Fig. 8 features the related reheating temperatures (cf. Eq. (115) instead. Again, both figures consists of three panels each that respectively take care of the the three different $B-L$ breaking scales specified in Eq. 102). We refer the interested reader to Appendix D which gives a detailed account of how Figs. 7 and 8 can be reconstructed by means of simple analytic expressions and with the aid of our numerical findings for $T_{R H}$ and $\eta_{B}$. 

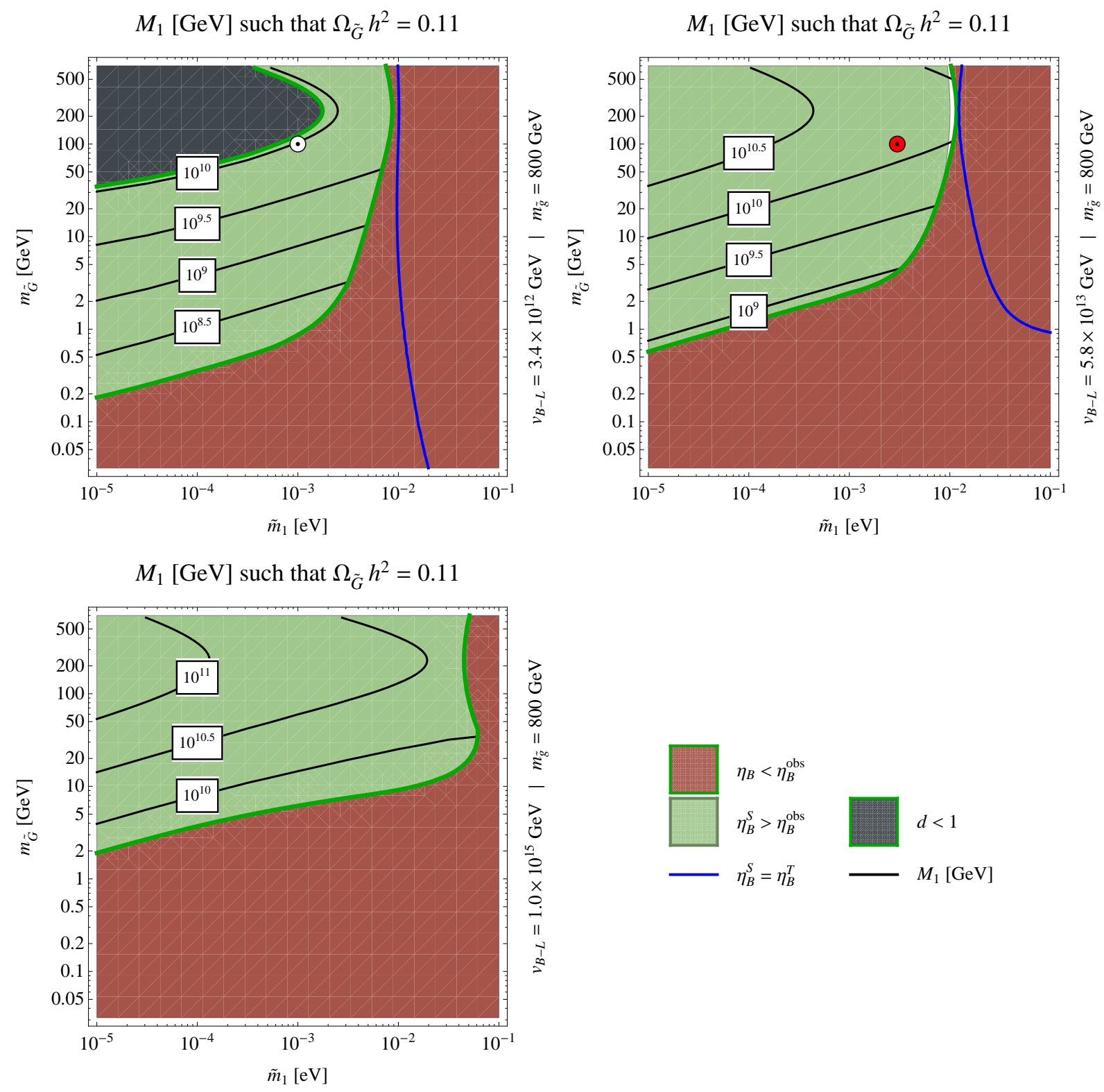

Figure 7: Gravitino mass range consistent with gravitino dark matter (cf. Eq. 112) and successful leptogenesis (cf. Eq. 116]) depending on the effective light neutrino mass. The contour lines refer to the neutrino mass $M_{1}$ (cf. Eq. (114) ) as a function of $\widetilde{m}_{1}$ and $m_{\widetilde{G}}$ such that the gravitino abundance is $\Omega_{\widetilde{G}} h^{2}=0.11$. In addition to the colour code introduced in Fig. 6, the black region in the upper-left panel represents the $M_{1}$ values that are not allowed by the flavour model. 

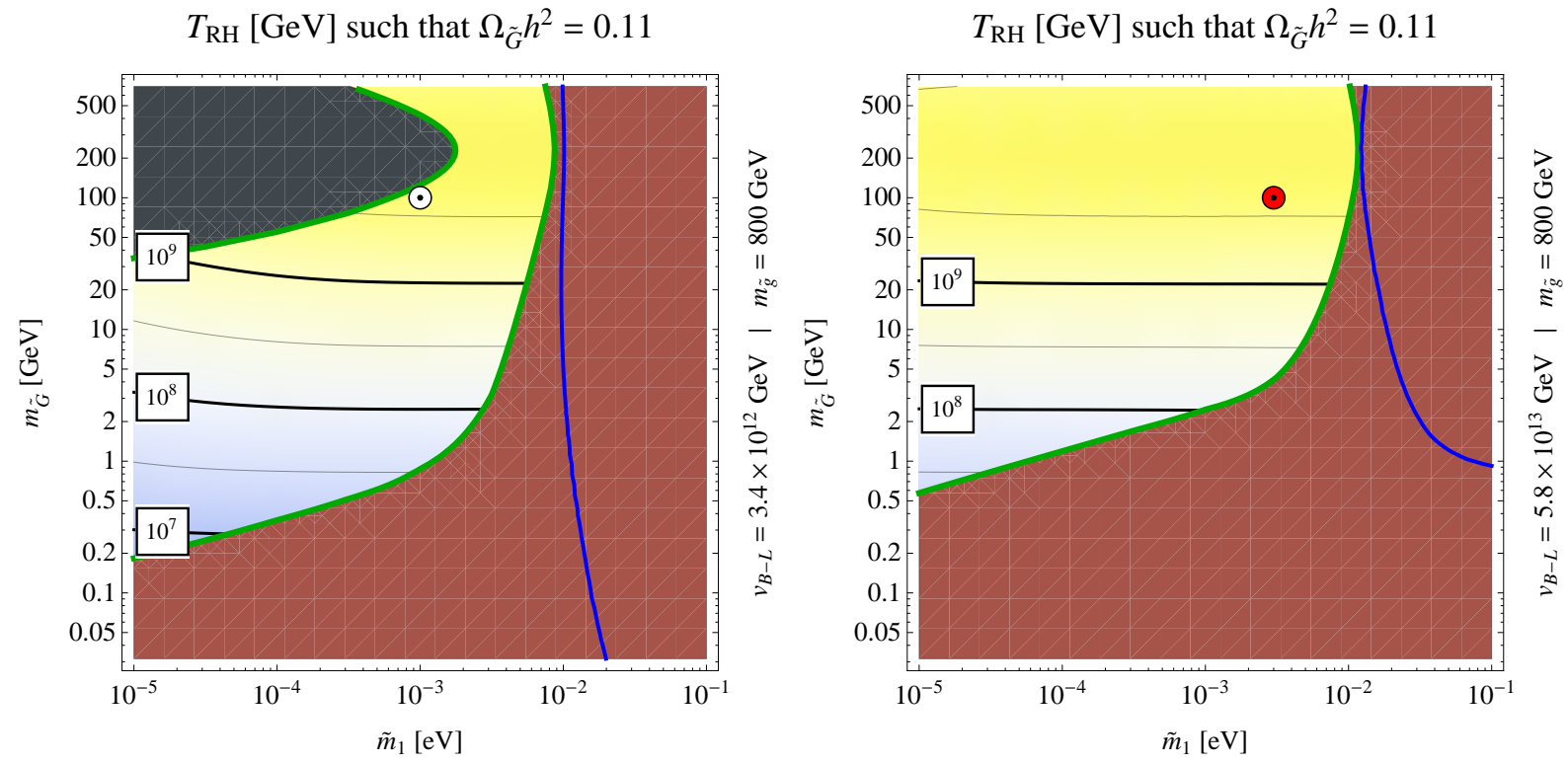

$T_{\mathrm{RH}}[\mathrm{GeV}]$ such that $\Omega_{\tilde{G}} h^{2}=0.11$

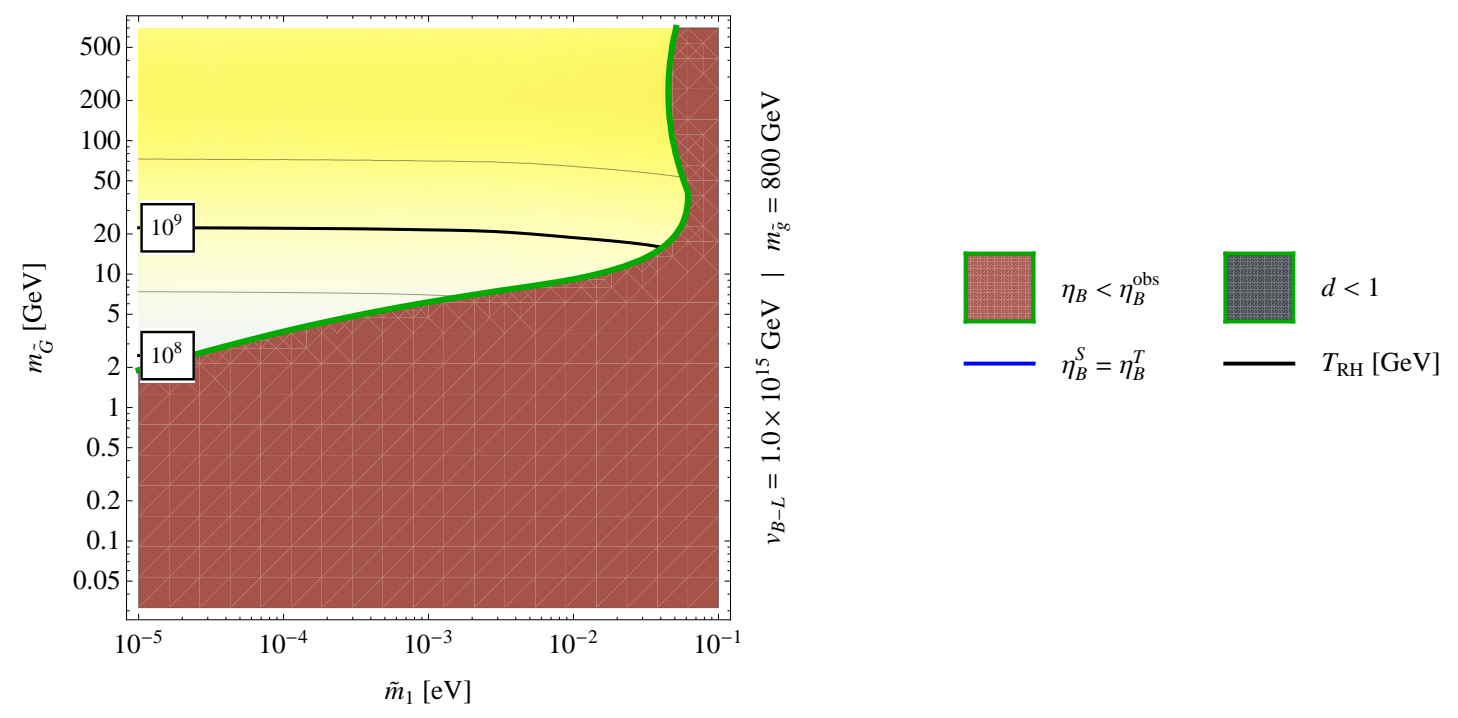

Figure 8: Like Fig.7 but with contours for the reheating temperature $T_{R H}$ (cf. Eq.115) instead of the neutrino mass $M_{1}$. 
Notice that we also consider gravitino masses almost as large as the gluino mass, $m_{\tilde{G}} \leq 700 \mathrm{GeV}$ while $m_{\tilde{g}}=800 \mathrm{GeV}$. Imposing gaugino mass unification at the GUT scale would, however, forbid such a nearly degenerate superparticle spectrum. The running of the renormalization group equations would then imply a gaugino mass relation $M_{3} / M_{1} \simeq$ 5.9 at low energies. Given that it is the lightest supersymmetric particle, the gravitino would have to be lighter than the bino resulting in an upper mass bound of $m_{\widetilde{G}} \lesssim 140 \mathrm{GeV}$.

If we were to select a gluino mass other than $m_{\tilde{g}}=800 \mathrm{GeV}$ all values of $m_{\tilde{G}}$ in Figs. 7 and 8 would have to be rescaled while $v_{B-L}, M_{1}$ and $\widetilde{m}_{1}$ could remain unchanged. This follows from the fact that the gravitino abundance in Eq. (112) can be kept constant by compensating a change $m_{\tilde{g}} \rightarrow a m_{\tilde{g}}$ in the gluino mass by a change $m_{\widetilde{G}} \rightarrow b m_{\widetilde{G}}$ in the gravitino mass without altering the reheating temperature $T_{R H}$. As long as $m_{\widetilde{G}}$ is much smaller than $m_{\tilde{g}}$, the factor $b$ simply corresponds to $a^{2}$. The general relation between $a$ and $b$ is discussed in Appendix D. To sum up, thanks to this relation the results presented in Figs. 7 and 8 can be generalized to different gluino masses by correspondingly relabeling the gravitino axis.

As a general trend in Figs. 77 and 8 we observe that for fixed $\widetilde{m}_{1}$ and $m_{\widetilde{G}} \lesssim 230 \mathrm{GeV}$ the neutrino mass $M_{1}$ and the reheating temperature $T_{R H}$ continuously become larger when increasing the gravitino mass. For $m_{\widetilde{G}} \gtrsim 230 \mathrm{GeV}$ this behaviour is reversed: The $M_{1}$ contours in Fig.7 bend over as soon as $m_{\widetilde{G}} \simeq 230 \mathrm{GeV}$ is exceeded. In Fig. 8 the temperature remains rather constant at $T_{R H} \sim 5 \times 10^{9} \mathrm{GeV}$ for $150 \mathrm{GeV} \lesssim m_{\widetilde{G}} \lesssim 400 \mathrm{GeV}$. Beyond $m_{\widetilde{G}} \gtrsim 400 \mathrm{GeV}$ it begins to decrease again. 9 The physical origin of these two regimes can be traced back to the rate $\Gamma_{\widetilde{G}}=\gamma_{\widetilde{G}} / n_{\widetilde{G}}$ (cf. Eqs. (85) and (86)) at which gravitinos are created from the thermal bath (cf. Eq. (D.6)) [14]15],

$$
\Gamma_{\widetilde{G}}=\Gamma_{\widetilde{G}}\left(T, m_{\widetilde{G}}, m_{\tilde{g}}\right) \propto\left(1+\frac{m_{\tilde{g}}^{2}(T)}{3 m_{\widetilde{G}}^{2}}\right) .
$$

In the regime $m_{\widetilde{G}} \ll m_{\tilde{g}}(T)$ the second term in Eq. (117) is the dominant one and it is mainly the goldstino part of the gravitino, i.e. its components with helicity $\pm \frac{1}{2}$, that is produced. A larger gravitino mass then implies a smaller rate $\Gamma_{\widetilde{G}}$ necessitating a stronger reheating in order to still generate the right abundance. Correspondingly, the neutrino mass $M_{1}$ also has to increase to bring about the higher temperature. Evolving a gluino mass of $800 \mathrm{GeV}$ from the electroweak scale to a temperature $T \sim 5 \times 10^{9} \mathrm{GeV}$ results in a high-scale mass of $m_{\tilde{g}}(T) \sim 400 \mathrm{GeV}$. Because of that, $\Gamma_{\widetilde{G}}$ is dominated by the first term in Eq. 117) from $m_{\widetilde{G}} \simeq 400 / \sqrt{3} \mathrm{GeV} \simeq 230 \mathrm{GeV}$ onwards. This means that, for

\footnotetext{
${ }^{9} \mathrm{Cf}$. the contour corresponding to $T_{R H}=10^{9.5} \mathrm{GeV}$ reentering the second panel of Fig. 8 at $\widetilde{m}_{1} \simeq$ $10^{-5} \mathrm{eV}$ and $m_{\widetilde{G}} \simeq 700 \mathrm{GeV}$.
} 
such large values of $m_{\tilde{G}}$, primarily the transverse degrees of freedom of the gravitino, i.e. its components with helicity $\pm \frac{3}{2}$, are excited. The production rate $\Gamma_{\widetilde{G}}$ then becomes independent of $m_{\widetilde{G}}$ turning into a function of the temperature $T$ only. In such a case the final gravitino abundance $\Omega_{\widetilde{G}} h^{2}$ simply scales linearly with $m_{\widetilde{G}}$ (cf. Eq. (D.1)). Hence, larger gravitino masses have to be balanced by smaller reheating temperatures to keep $\Omega_{\widetilde{G}} h^{2}$ fixed. This explains the decrease in $T_{R H}$ and $M_{1}$ at very large gravitino masses.

On the other hand, varying $\widetilde{m}_{1}$ at constant $m_{\widetilde{G}}$ has almost no effect on the reheating temperature, which is expected since $\Gamma_{\widetilde{G}}$ inherently is a function of $T, m_{\widetilde{G}}$ and $m_{\tilde{g}}$. As each gravitino mass is associated with an appropriate rate $\Gamma_{\widetilde{G}}$, the choice of $m_{\widetilde{G}}$ already implies a unique reheating temperature $T_{R H} \approx T_{R H}\left(m_{\widetilde{G}}\right)$, independent of the underlying neutrino parameters (cf. Fig. 12 in Appendix D). Meanwhile, the neutrino mass $M_{1}$ becomes smaller when increasing $\widetilde{m}_{1}$ in order to ensure that $T_{R H}$ remains approximately constant for fixed $m_{\widetilde{G}}$ (cf. Fig. 5).

In Figs. 7 and 8 we also indicate the regions in parameter space that are not compatible with our scenario because either of the two conditions in Eqs. (114) and (116) is not satisfied. Bounds coming from the flavour model (cf. Eq. (114)) only show up for $v_{B-L}=$ $3.4 \times 10^{12} \mathrm{GeV}$ : The requirement that $M_{1}$ be smaller than $1.1 \times 10^{10} \mathrm{GeV}$ (cf. Eq. (102)) excludes gravitino masses larger than $35 \mathrm{GeV}$ for $\widetilde{m}_{1}=10^{-5} \mathrm{eV}$. At $\widetilde{m}_{1}=10^{-3} \mathrm{eV}$ it rules out masses in the range between $120 \mathrm{GeV}$ and $430 \mathrm{GeV}$ and for $\widetilde{m}_{1} \gtrsim 1.8 \times 10^{-3} \mathrm{eV}$ it does not constrain $m_{\widetilde{G}}$ any longer at all. In the case of the two other choices for $v_{B-L}$ the respective flavour bounds on $M_{1}$ are never reached because the corresponding reheating temperatures are too high. Demanding a sufficient baryon asymmetry (cf. Eq. 1116) yields lower bounds on $m_{\widetilde{G}}$ in the weak washout regime and limits the maximal value of $\widetilde{m}_{1}$. Notice that these bounds are in one-to-one correspondence with the constraints on $M_{1}$ and $\widetilde{m}_{1}$ in Fig.6. For instance, at small $\widetilde{m}_{1}$ the gravitino mass can only decrease as long as $M_{1}$ is large enough so that the observed baryon asymmetry is reproduced. Similarly, at large $\widetilde{m}_{1}$ the sharp drop-off in the efficiency of leptogenesis due to stronger washout limits the viable range of $\widetilde{m}_{1}$. In Tab. 4 we present the smallest gravitino masses that are accessible for certain representative values of $\widetilde{m}_{1}$. As in Fig. 7 the contour lines of constant $M_{1}$ slightly fall off with decreasing $\widetilde{m}_{1}$, we find the lowest bounds on $m_{\widetilde{G}}$ at $\widetilde{m}_{1}=10^{-5} \mathrm{eV}$. For weak washout higher $B-L$ breaking scales lead to tighter bounds on $m_{\widetilde{G}}$, just as it is the case for the neutrino mass $M_{1}$ (cf. Fig.6). In the strong washout regime we encounter the opposite behavior. Here, the contour line corresponding to $\eta_{B}=\eta_{B}^{\text {obs }}$, which separates the allowed and excluded regions in parameter space, steeply rises. In Section 5.1 we argued that the larger the value of $v_{B-L}$ the later this rise sets in when increasing $\widetilde{m}_{1}$ (cf. Eq. (109)). Therefore, the tightest bounds on $m_{\widetilde{G}}$ are now obtained for low $B-L$ 


\begin{tabular}{ccccccc} 
Panel & $v_{B-L}[\mathrm{GeV}] / \widetilde{m}_{1}[\mathrm{eV}]$ & $10^{-5}$ & $10^{-4}$ & $10^{-3}$ & $10^{-2}$ & $10^{-1}$ \\
\hline \hline 1 & $3.4 \times 10^{12}$ & $180 \mathrm{MeV}$ & $360 \mathrm{MeV}$ & $870 \mathrm{MeV}$ & - & - \\
2 & $5.8 \times 10^{13}$ & $570 \mathrm{MeV}$ & $1.2 \mathrm{GeV}$ & $2.5 \mathrm{GeV}$ & $70 \mathrm{GeV}$ & - \\
3 & $1.0 \times 10^{15}$ & $1.9 \mathrm{GeV}$ & $3.7 \mathrm{GeV}$ & $6.2 \mathrm{GeV}$ & $9.2 \mathrm{GeV}$ & - \\
\hline \hline
\end{tabular}

Table 4: Lower bounds on the gravitino mass according to Figs. 7 and 8 for the three different choices of $v_{B-L}$ listed in Eq. 102 and five different values of $\widetilde{m}_{1}$. A dash (-) indicates that leptogenesis is not efficient enough to produce the observed baryon asymmetry as long as the requirement of gravitino dark matter is kept.

breaking scales.

We also note that for $\widetilde{m}_{1}=0.1 \mathrm{eV}$ it is not possible to produce the observed baryon asymmetry while sticking to the assumption of gravitino dark matter, independent of the value chosen for $v_{B-L}$. As $\widetilde{m}_{1}$ is bounded from below by $m_{1}$, the smallest eigenvalue of the standard model neutrino mass matrix, this observation opens up the possibility of falsifying our proposed scenario in future neutrino experiments. The measurement of a light neutrino mass of $0.1 \mathrm{eV}$, combined with the known differences of the light neutrino masses squared, would imply that $\widetilde{m}_{1} \gtrsim 0.1 \mathrm{eV}$, thereby ruling out our mechanism of entropy production. Likewise, any lower limit on the absolute neutrino mass scale coming from, e.g. cosmological observations would restrict the allowed range for the gravitino mass. A determination of the gravitino mass on the basis of cosmic gamma-ray observations or decays of the next-to-lightest-superparticle (NLSP) in collider experiments could, in turn, constrain the neutrino mass spectrum.

In standard thermal leptogenesis the reheating temperature has to be at least $T_{R H} \gtrsim$ $10^{9} \mathrm{GeV}$, independent of the initial conditions, to guarantee a successful generation of the baryon asymmetry [33. Together with the lower bound on the gluino mass imposed by collider searches, this constrains the gravitino mass to lie in the range $m_{\widetilde{G}} \simeq 10 \div 100 \mathrm{GeV}$ in order to be compatible with the observed dark matter abundance. By contrast, the present scenario allows for a much broader range of gravitino masses since the reheating temperature can be significantly lower than in the case of thermal leptogenesis. As apparent in Fig. 8, $T_{R H}$ can decrease down to values of $\mathcal{O}\left(10^{7}\right) \mathrm{GeV}$ if $\widetilde{m}_{1}$ and $M_{1}$ are chosen such that the nonthermal neutrinos decay extremely slowly. We note that for such reheating temperature production of gravitinos from inflaton decay is usually negligible [38]. This paves the way for gravitino masses as small as $200 \mathrm{MeV}$ (cf. Tab. (4). On the other hand, as our scenario can as well accomodate neutrino masses $M_{1}$ of order $10^{10} \div 10^{11} \mathrm{GeV}$, the gravitino can also be almost as heavy as the gluino, $m_{\widetilde{G}} \simeq$ few $\times 100 \mathrm{GeV}$. Note, 
however, that requirements such as gaugino mass unification will further constrain the superparticle spectrum.

Finally, we would like to point out that low gravitino masses have interesting consequences for the decay of the next-to-lightest superparticle (NLSP). If $R$-parity is conserved, the lower bound on the mass of stable gravitinos from standard thermal leptoge-

nesis, $m_{\widetilde{G}} \gtrsim 10 \mathrm{GeV}$, implies a long NLSP lifetime which could jeopardize the success of primordial nucleosynthesis (BBN).

A study of general neutralino NLSPs with gravitino LSP has been performed in [39]. In this work, lower mass bounds for different NLSP types have been extracted from the bounds on the decay of heavy neutral particles during BBN for given gravitino masses. Assuming a gravitino of $10 \mathrm{GeV}$, it turns out that the predictions of primordial nucleosynthesis are not affected for pure bino, pure wino and mixed gaugino-Higgsino neutralino NLSPs that are heavier than 3, 0.8 and $1 \mathrm{TeV}$, respectively. These bounds are now significantly softened for the light gravitino masses which can occur in our scenario: For $m_{\widetilde{G}}=200 \mathrm{MeV}$, pure bino, pure wino and mixed gaugino-Higgsino neutralino NLSPs as light as 800,150 and $200 \mathrm{GeV}$, respectively, are in agreement with the BBN bounds. These lower masses are interesting for two reasons: First, they allow a smaller scale of supersymmetry breaking and second, they can be probed more easily at the LHC. For a charged NLSP like a scalar $\tau$-lepton, its lifetime has to be sufficiently short, $\tau_{\mathrm{NSLP}} \lesssim 10^{3}-10^{4} \mathrm{~s}$ [0,41], which typically requires $m_{\widetilde{G}}<1 \mathrm{GeV}$ for $m_{\mathrm{NLSP}}=\mathcal{O}(100) \mathrm{GeV}$.

Remarkably, the small gravitino masses required from such constraints on NLSP decays can be accommodated in our scenario. We thus emphasize that reheating through the decays of heavy neutrinos provides a way to alleviate the existing tension between the generation of the baryon asymmetry, stable gravitino dark matter and BBN constraints.

\section{$6 \quad$ Summary and outlook}

We have studied the production of the entropy of the hot early universe through the decays of heavy Majorana neutrinos. As an example, we considered the decay of a false vacuum with unbroken $B-L$ symmetry. Tachyonic preheating leads to a state whose energy density is dominated by nonrelativistic $S$ bosons, the Higgs boson associated with spontaneous $B-L$ breaking, with a subdominant admixture of radiation. Subsequent production of the lightest heavy Majorana neutrinos $N_{1}$ from $S$ decays and from the thermal bath leads to a phase whose energy density is dominated by $N_{1}$ neutrinos. Their decay then produces all entropy of the hot thermal universe, together with the baryon asym- 
metry via a mixture of nonthermal and thermal leptogenesis. Simultaneously, thermal production of gravitinos accounts for the observed dark matter.

We have studied the time evolution of this system by means of a set of Boltzmann equations for distribution functions which take into account the differences between thermally and nonthermally produced $N_{1}$ neutrinos. Details of the initial state are rather unimportant for the final result. What matters is the existence of a phase dominated by $N_{1}$ neutrinos. Their lifetime determines the reheating temperature that is crucial for the final gravitino abundance and hence for the produced amount of dark matter.

Our analysis has been based on a flavour model that allows to vary the key parameters for leptogenesis, the neutrino masses $M_{1}$ and $\widetilde{m}_{1}$, over a wide range, consistent with the masses and flavour mixings of charged leptons and neutrinos. The final baryon asymmetry and the dark matter abundance have been calculated in terms of several parameters of the Lagrangian, independent of initial conditions: the scale $v_{B-L}$ of $B-L$ breaking, the

heavy Majorana neutrino mass $M_{1}$, the effective light neutrino mass $\widetilde{m}_{1}$, the gravitino mass $m_{\tilde{G}}$ and the gluino mass $m_{\tilde{g}}$. For generalisations of the flavour model the produced matter-antimatter asymmetry and dark matter can depend on further parameters.

Particularly interesting is the resulting relation between the lightest neutrino mass and the gravitino mass. For instance, for a typical gluino mass of $800 \mathrm{GeV}$ and a light neutrino mass of $10^{-5} \mathrm{eV}$ the gravitino mass can be as small as $200 \mathrm{MeV}$, whereas a lower neutrino mass bound of $0.01 \mathrm{eV}$ implies a lower bound of $9 \mathrm{GeV}$ on the gravitino mass. The measurement of a light neutrino mass of $0.1 \mathrm{eV}$ would falsify the proposed mechanism of entropy production. These results provide an important connection between collider searches for superparticles and neutrino mass determinations in laboratory experiments and by cosmological observations. Measurements of the absolute neutrino mass scale and superparticle masses consistent with our predictions would provide important indirect evidence for the origin of entropy, matter and dark matter and for the maximal temperature of the hot thermal universe.

We are planning to extend our analysis in several directions: Boltzmann equations for the superpartners of $S$ bosons and heavy Majorana neutrinos have to be incorporated in a fully consistent calculation of baryon asymmetry and gravitino dark matter. Further important questions concern the connection with inflation and the possible relation between $B-L$ breaking and supersymmetry breaking.

\section{Acknowledements}

The authors thank L. Covi, V. Domcke and J. Hasenkamp for helpful discussions. This 
work has been supported by the German Science Foundation (DFG) within the Collaborative Research Center 676 "Particles, Strings and the Early Universe".

\section{A Conventions for the Boltzmann equations}

The Boltzmann equation for a particle species $\psi$ describes the time evolution of the distribution function $f_{\psi}\left(t, x^{\mu}, p^{\mu}\right)$ in the one-particle phase space $\Phi_{\psi}$ [37], where $f_{\psi}$ is defined such that $f_{\psi} d \Phi_{\psi}$ gives the average number of $\psi$ particles in the phase space volume $d \Phi_{\psi}$ at time $t$. Imposing homogeneity and isotropy of spacetime, $f_{\psi}$ only depends on the absolute value $p$ of the 3 -momentum $\vec{p}$ as well as on time $t$. The Boltzmann equation for $\psi$ particles in the Friedmann-Lemaitre framework then reads

$$
\hat{\mathcal{L}} f_{\psi}(t, p)=\left(\frac{\partial}{\partial t}-H p \frac{\partial}{\partial p}\right) f_{\psi}(t, p)=\mathcal{C}_{\psi},
$$

where with $\hat{\mathcal{L}}$ we denote the Liouville operator, and with $\mathcal{C}_{\psi}$ the collision operator. The latter keeps track of changes in $f_{\psi}$ due to inelastic interactions, and may be decomposed into contributions from decays and scatterings,

$$
\mathcal{C}_{\psi}=\sum_{i j \ldots} C_{\psi}(\psi \leftrightarrow i j \ldots)+\sum_{a} \sum_{i j \ldots} C_{\psi}(\psi a \leftrightarrow i j \ldots)+\ldots
$$

The operators $C_{\psi}$ are obtained from quantum mechanical transition probabilities integrated over the multi-particle phase space

$$
\begin{aligned}
C_{\psi}(\psi a b \ldots \leftrightarrow i j \ldots)= & \frac{1}{2 g_{\psi} E_{\psi}} \int d \Pi(\psi \mid a, b, \ldots ; i, j, \ldots)(2 \pi)^{4} \delta^{(4)}\left(\sum p_{\text {out }}-\sum p_{\text {in }}\right) \\
& \times\left[f_{i} f_{j} \ldots\left(1 \pm f_{\psi}\right)\left(1 \pm f_{a}\right)\left(1 \pm f_{b}\right) \ldots|\mathcal{M}(i j \ldots \rightarrow \psi a b \ldots)|^{2}\right. \\
& \left.-f_{\psi} f_{a} f_{b} \ldots\left(1 \pm f_{i}\right)\left(1 \pm f_{j}\right) \ldots|\mathcal{M}(\psi a b \ldots \rightarrow i j \ldots)|^{2}\right],
\end{aligned}
$$

where $g_{\psi}$ is the number of internal degrees of freedom of $\psi$ and $d \Pi$ subsumes all Lorentz invariant momentum space elements $d \tilde{p}=(2 \pi)^{-3} d^{3} p / 2 E$ along with a statistical factor $S$ that prevents double counting in the case of identical particles

$$
d \Pi(\psi \mid a, b, \ldots ; i, j, \ldots)=S(\psi, a, b, \ldots ; i, j, \ldots) d \tilde{p}_{a} d \tilde{p}_{b} \ldots d \tilde{p}_{i} d \tilde{p}_{j} \ldots
$$

The amplitudes squared $|\mathcal{M}|^{2}$ are understood to be summed over all internal degrees of freedom. Since they are expected to yield only small corrections [34, the Bose enhancement $(1+f)$ and Pauli blocking $(1-f)$ quantum statistical factors related to boson and fermion production respectively, are neglected in this work. Their influence may partly 
be canceled by other quantum corrections, like off-shell effects [42]. Additionally, the $C_{\psi}$ operators can be split into direct and inverse processes

$$
C_{\psi}(\psi a b \ldots \leftrightarrow i j \ldots)=C_{\psi}(i j \ldots \rightarrow \psi a b \ldots)-C_{\psi}(\psi a b \ldots \rightarrow i j \ldots) .
$$

If the $\psi$ particles are in kinetic equilibrium, the integration of Eq. A.1] over the $\psi$ phase space leads to a Boltzmann equation for the $\psi$ number density $n_{\psi}$

$$
\dot{n}_{\psi}+3 H n_{\psi}=\sum_{i j \ldots} \gamma(\psi \leftrightarrow i j \ldots)+\sum_{a} \sum_{i j \ldots} \gamma(\psi a \leftrightarrow i j \ldots)+\ldots,
$$

where $n_{\psi}$ and the interaction densities $\gamma$ are defined as

$$
\begin{aligned}
n_{\psi}(t) & =\frac{g_{\psi}}{(2 \pi)^{3}} \int d^{3} p f_{\psi}(t, p), \\
\gamma(\psi a b \ldots \leftrightarrow i j \ldots) & =\frac{g_{\psi}}{(2 \pi)^{3}} \int d^{3} p C_{\psi}(\psi a b \ldots \leftrightarrow i j \ldots) .
\end{aligned}
$$

The Boltzmann equation Eq. (A.6) can alternatively be written as an equation for the comoving number density $N_{\psi}=a^{3} n_{\psi}$ as a function of the scale factor $a$

$$
a H \frac{d}{d a} N_{\psi}=a^{3}\left[\sum_{i j \ldots} \gamma(\psi \leftrightarrow i j \ldots)+\sum_{a} \sum_{i j \ldots} \gamma(\psi a \leftrightarrow i j \ldots)+\ldots\right] .
$$

\section{B Phase space distribution of thermal neutrinos}

When deriving the Boltzmann equation (69) for thermal neutrinos in Section 3.2.3, we asserted that these are approximately in kinetic equilibrium (cf. Eq. (68)). Given a numerical solution for $T(a)$ for a specific choice of input parameters $v_{B-L}, \widetilde{m}_{1}$ and $M_{1}$, we can check the self-consistency of this approach by comparing our approximate distribution function proportional to $f_{N_{1}}^{\text {eq }}$ with the exact expression in Eq. (67). In this appendix we perform such a comparison for the exemplary parameter point discussed in Section 4 (cf. Tab. 2). To begin with, we introduce the following ratio

$$
\begin{aligned}
R_{N_{1}}^{T}(t, p) & =\frac{f_{N_{1}}^{T}(t, p)}{f_{N_{1}}^{\mathrm{eq}}(t, p)} \\
& =\int_{t_{2}}^{t} d t^{\prime} \exp \left(-M_{1} \Gamma_{N_{1}}^{0} \int_{t^{\prime}}^{t} d t^{\prime \prime} E_{N_{1}}^{-1}\left(t^{\prime \prime}\right)\right) \frac{M_{1}}{E_{N_{1}}\left(t^{\prime}\right)} \Gamma_{N_{1}}^{0} \frac{f_{N_{1}}^{\mathrm{eq}}\left(t^{\prime}, p\right)}{f_{N_{1}}^{\mathrm{eq}}(t, p)} .
\end{aligned}
$$




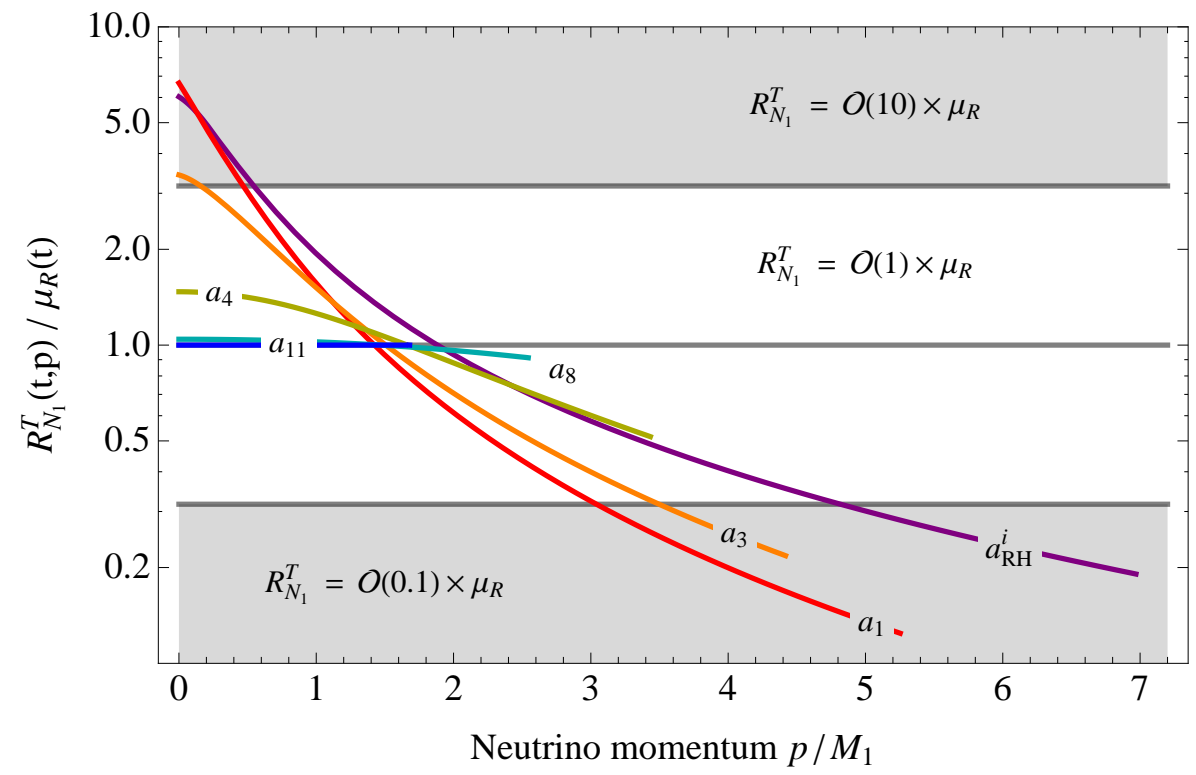

Figure 9: Ratio $R_{N_{1}}^{T}=f_{N_{1}}^{T} / f_{N_{1}}^{\text {eq }}$ normalized to its mean value $\mu_{R}$ as a function of the neutrino momentum $p$ at different times, cf. Eqs. (B.2) and (B.3) as well as Tab. 5 . The input parameters $v_{B-L}, \widetilde{m}_{1}$ and $M_{1}$ are chosen as in Section 4 (cf. Tab. 2). The respective values of the scale factor are discussed in Section 4.2 . The white and gray bands separate the different orders of magnitude.

with $f_{N_{1}}^{T}$ taken from Eq. 67), and determine its momentum dependence at different times. A momentum independence of $R_{N_{1}}^{T}$ would then reflect an exact kinetic equilibrium. In this case the equation $f_{N_{1}}^{T}=R_{N_{1}}^{T} f_{N_{1}}^{\text {eq }}$ can easily be integrated over phase space yielding

$$
R_{N_{1}}^{T}=R_{N_{1}}^{T}(t) \quad \Leftrightarrow \quad N_{N_{1}}^{T}(t)=R_{N_{1}}^{T}(t) N_{N_{1}}^{\mathrm{eq}}(t) \quad \Leftrightarrow \quad f_{N_{1}}^{T}(t, p)=\frac{N_{N_{1}}^{T}(t)}{N_{N_{1}}^{\mathrm{eq}}(t)} f_{N_{1}}^{\mathrm{eq}}(t, p) .
$$

A convenient measure for the deviation from kinetic equilibrium at a given time is the coefficient of variation $c_{R}=\sigma_{R} / \mu_{R}$, i.e. the standard deviation $\sigma_{R}$ of $R_{N_{1}}^{T}$ in relation to its mean value $\mu_{R}$ with respect to an appropriate momentum interval $\Delta p$,

$$
\mu_{R}(t)=\left\langle R_{N_{1}}^{T}\right\rangle_{p}, \quad \sigma_{R}(t)=\left(\left\langle\left(R_{N_{1}}^{T}\right)^{2}\right\rangle_{p}-\left\langle R_{N_{1}}^{T}\right\rangle_{p}^{2}\right)^{1 / 2}
$$

where $\langle\cdot\rangle_{p}$ is defined as

$$
\langle f\rangle_{p}(t)=\frac{1}{\Delta p} \int_{0}^{\Delta p} d p f(t, p),
$$

and $\Delta p$, by convention, is always chosen as

$$
f_{N_{1}}^{\mathrm{eq}}(t, \Delta p)=10^{-4} \times f_{N_{1}}^{\mathrm{eq}}(t, 0)
$$


such that the relevant range of momenta is covered.

We compute $R_{N_{1}}^{T}, \mu_{R}, \sigma_{R}, c_{R}$ and $\Delta p$ for six representative values of the scale factor, and summarize the corresponding results in Fig. 9 and Table 5. At early times small momenta are much more frequent than in kinetic equilibrium and states with large momenta are underpopulated. For extreme momenta, $R_{N_{1}}^{T}$ can become ten times as large or small as its mean value $\mu_{R}$. As time goes on, this tilt in $R_{N_{1}}^{T}$, however, disappears and for $a \simeq 12000$, kinetic equilibrium is eventually reached. On average $R_{N_{1}}^{T}$ deviates from $\mu_{R}$ not more than one order of magnitude and, from this perspective, the approximation of kinetic equilibrium may be regarded as justified. The steady convergence to kinetic equilibrium is also reflected in the behaviour of the coefficient of variation $c_{R}$ which starts out at a value of $c_{R} \sim \mathcal{O}(1)$ and decreases to $c_{R} \sim \mathcal{O}\left(10^{-4}\right)$. For other choices of the model parameters we expect the $N_{1}^{T}$ phase space distribution to behave similarly.

Scatterings of the thermal neutrinos involving standard model quark pairs such as $N_{1} \ell \leftrightarrow q \bar{u}, N_{1} \bar{u} \leftrightarrow \ell \bar{q}$ and $N_{1} q \leftrightarrow \ell u$, speed up the equilibration of the neutrino distribution function [34. This results in a larger abundance of thermal neutrinos at high temperatures. On the other hand, scatterings also tend to increase the efficiency of washout processes such that, after all, their impact on the generated thermal asymmetry is negligible for our purposes.

There are two main reasons why $R_{N_{1}}^{T}$ is not flat from the beginning: The first is directly related to the momentum dependence of the production and decay terms in the Boltzmann equation (69) for thermal neutrinos. In both terms the effective rate $\Gamma_{N_{1}}^{T}$ comes weighted with the inverse time dilatation factor $\left\langle M_{1} / E_{N_{1}}\right\rangle$. It is, thus, larger at smaller momenta which is why initially, when $f_{N_{1}}^{T} \ll f_{N_{1}}^{\text {eq }}$, neutrinos with smaller momenta are overproduced. Once $f_{N_{1}}^{T}$ has overshot $f_{N_{1}}^{\text {eq }}$ the decay term dominates, again preferably changing the abundance of low-momentum states. This interplay between production and decay is balanced such that $R_{N_{1}}^{T}$ is eventually flattened out. A numerical investigation of the different factors in the integrand of Eq. (B.1) confirms this simple argument. The second reason is the intermediate stage of reheating between the phases of adiabatic expansion. Assuming an equilibrium distribution $f_{N_{1}}^{\text {eq }}$ misconceives the evolution of the temperature in the sense that higher temperatures and thus more high-momentum neutrinos are expected at earlier times. By contrast, the actual distribution $f_{N_{1}}^{T}$ takes the temperature plateau into account and is, hence, aware of the correspondingly less efficient production at high momenta. 


\begin{tabular}{cccccc}
$\#$ & $a$ & $\mu_{R}$ & $\sigma_{R}$ & $c_{R}$ & $\Delta p\left[M_{1}\right]$ \\
\hline \hline 1 & 27 & $8.4 \times 10^{-4}$ & $1.0 \times 10^{-3}$ & 1.2 & 7.0 \\
2 & 210 & $1.0 \times 10^{-2}$ & $1.4 \times 10^{-2}$ & 1.3 & 5.3 \\
3 & 1500 & 0.29 & 0.25 & 0.87 & 4.4 \\
4 & 3500 & 0.75 & 0.24 & 0.32 & 3.4 \\
5 & 6500 & 1.0 & $4.0 \times 10^{-2}$ & $3.9 \times 10^{-2}$ & 2.5 \\
6 & 12000 & 1.0 & $4.0 \times 10^{-5}$ & $3.9 \times 10^{-5}$ & 1.7 \\
\hline \hline
\end{tabular}

Table 5: Indicators for the deviation of the thermal neutrinos from kinetic equilibrium at different times: $\mu_{R}, \sigma_{R}$ and $\Delta p$ are introduced in Eqs. (B.3) and (B.5), $c_{R}$ is defined as $c_{R}=\sigma_{R} / \mu_{R}$. The values of the scale factor correspond to $a_{R H}^{i}, a_{1}, a_{3}, a_{4}, a_{8}$ and $a_{11}, c f$. Section 4.2 and Fig. 9 .

\section{Reheating temperature}

The energy transfer to the thermal bath, i.e. the reheating of the universe, becomes fully efficient when the nonthermally produced $N_{1}$ neutrinos decay into standard model radiation. This happens once the Hubble rate $H$ has dropped to the value of the effective decay rate $\Gamma_{N_{1}}^{S}$ of the nonthermal $N_{1}$ neutrinos. The temperature at this time, $t=t_{R H}$, defines the reheating temperature $T_{R H}$

$$
\Gamma_{N_{1}}^{S}\left(t_{R H}\right)=H\left(t_{R H}\right), \quad T_{R H}=T\left(t_{R H}\right) .
$$

Notice that this coincides with the common definition of $T_{R H}$ in scenarios in which the universe is reheated through the decay of some species with effective decay rate $\Gamma$.

The decay rate relevant to our scenario, $\Gamma_{N_{1}}^{S}$, corresponds to the $T=0$ neutrino decay width $\Gamma_{N_{1}}^{0}$ weighted with the average inverse time dilatation factor for nonthermal neutrinos (cf. Section 3.2.3)

$$
\Gamma_{N_{1}}^{S}=\gamma_{t}^{-1} \Gamma_{N_{1}}^{0}, \quad \gamma_{t}=\left\langle\frac{M_{1}}{E_{N_{1}}}\right\rangle_{S}^{-1}, \quad \gamma=\gamma_{t}\left(t_{R H}\right)
$$

In the course of our numerical analysis Eqs. C.1) and (C.2) are used to determine the reheating temperature as a function of the model parameters, $T_{R H}=T_{R H}\left(v_{B-L}, M_{1}, \tilde{m}_{1}\right)$. The result of this computation is presented in Fig. 5 in Section 5 . In this appendix we shall illustrate how one can understand the exact outcome of the Boltzmann equations in terms of increasingly accurate approximations (also shown in Fig. 5).

We begin with Eq. (C.1) and try to solve it analytically for $T_{R H}$. First, the Friedmann 
equation allows us to express $H\left(t_{R H}\right)$ through the total energy density at time $t_{R H}$

$$
H^{2}\left(t_{R H}\right)=\left(\frac{\dot{a}}{a}\right)_{t_{R H}}^{2}=\beta^{2} \frac{8 \pi}{3 M_{p}^{2}} \rho_{\mathrm{tot}}\left(t_{R H}\right) .
$$

Here $\beta$ is a correction factor that accounts for the fact that we do not determine the scale factor $a$ dynamically but simply approximate it by means of constant effective coefficients $\omega$ in the equation of state. This imprecision in $a$ is then transmitted to $H$ such that it does not fulfill the Friedmann equation exactly. Second, let us denote the fraction of the total energy density that is stored in radiaton at time $t_{R H}$ by $\alpha^{-1}$. With the aid of Eq. 27) we may then write

$$
H^{2}\left(t_{R H}\right)=\alpha \beta^{2} \frac{8 \pi}{3 M_{p}^{2}} \frac{\pi^{2}}{30} g_{\star, \rho} T_{R H}^{4} .
$$

Combining Eqs. (C.1), (C.2) and (C.4) one finds

$$
T_{R H}=\alpha^{-1 / 4} \beta^{-1 / 2} \gamma^{-1 / 2}\left(\frac{90}{8 \pi^{3} g_{\star, \rho}}\right)^{1 / 4} \sqrt{\Gamma_{N_{1}}^{0} M_{p}} .
$$

By construction this formula yields the same results as Eq. (C.1). Its main advantage over Eq. (C.1), however, is that it allows us to estimate $T_{R H}$ with varying precision depending on how accurately the correction factors $\alpha, \beta$ and $\gamma$ are taken into account.

When solving the Boltzmann equations numerically we have also determined these factors along the way. The dependence of the time dilatation factor $\gamma$ on the neutrino parameters is shown in Fig. 10. As all nonthermal neutrinos are produced with initial energy $\frac{1}{2} m_{S} \simeq \frac{1}{2} \eta^{-2} M_{1} \simeq 150 M_{1}$ it is clear that $\gamma$ is bounded from above: $1 \leq \gamma \lesssim 150$. In practice, we find that $\gamma$ takes on values roughly between 1.1 and 88 entailing $\gamma^{-1 / 2}$ factors in Eq. C.5 approximately between 0.95 and 0.11 . The general behaviour of $\gamma$ as a function of the model parameters is mainly controlled by the ratio of the $S$ and $N_{1}$ decay widths

$$
\gamma=\gamma\left(\Gamma_{N_{1}}^{0} / \Gamma_{S}^{0}\right), \quad \frac{\Gamma_{N_{1}}^{0}}{\Gamma_{S}^{0}} \propto \frac{v_{B-L}^{2} \widetilde{m}_{1}}{v_{E W}^{2} M_{1}} .
$$

The larger $\Gamma_{N_{1}}^{0}$ compared to $\Gamma_{S}^{0}$ the less contribute very long-lived nonthermal $N_{1}$ neutrinos to $\gamma$. Most nonthermal neutrinos present at $t=t_{R H}$ were then produced just shortly before and are hence relativistic. On the other hand, if the $S$ bosons decay very fast, $\Gamma_{S}^{0} \gg \Gamma_{N_{1}}^{0}$, the nonthermal neutrinos are mainly produced at the early stages of reheating and $\gamma$ is rather dominated by elder, nonrelativistic neutrinos. 

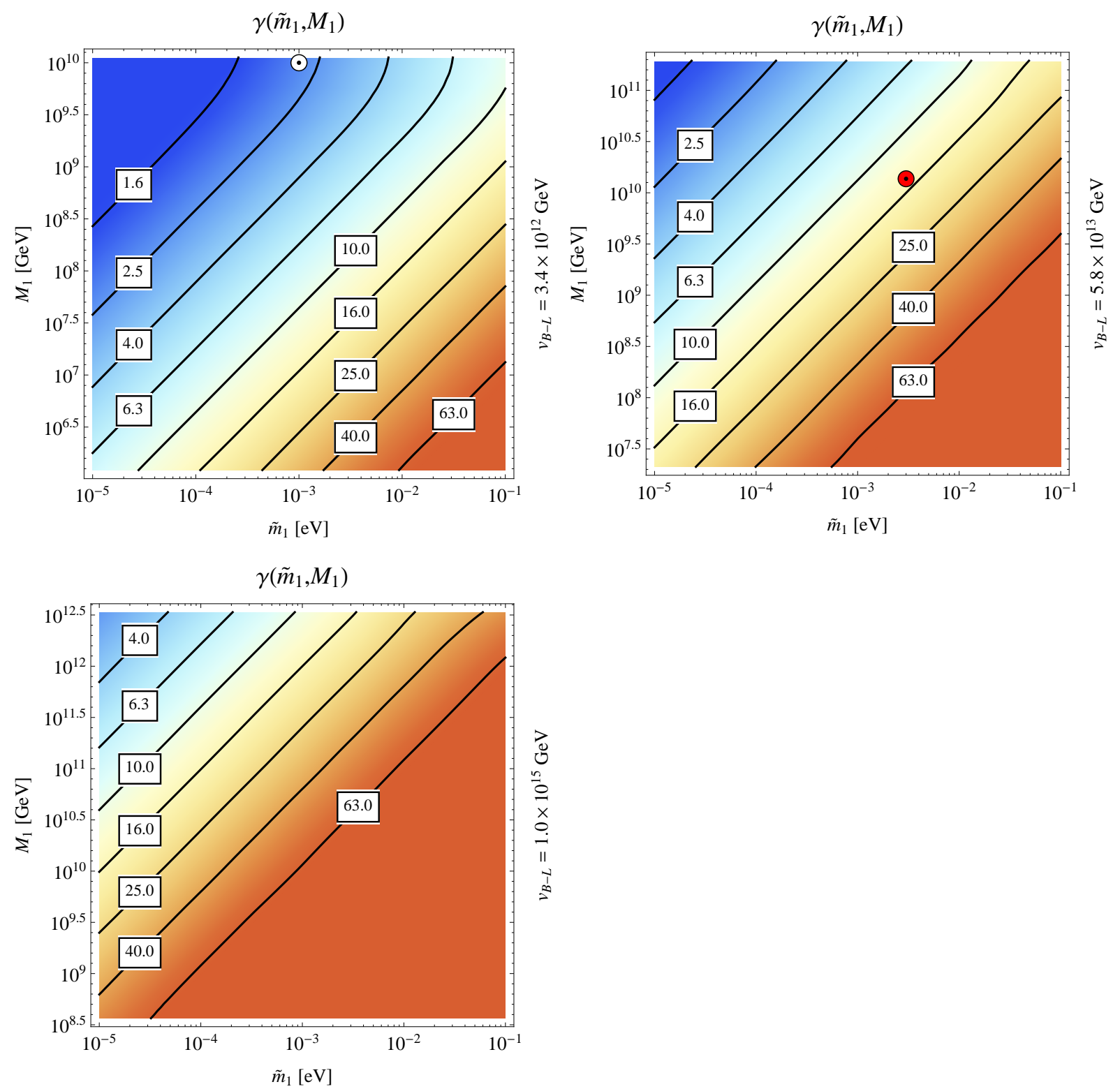

Figure 10: Contour plots of the relativistic correction factor $\gamma$ as a function of the parameters $\widetilde{m}_{1}$ and $M_{1}$ for the three different choices of $v_{B-L}$ (cf. Eq. 102). $\gamma^{-1}$ is defined as the average inverse time dilatation factor for nonthermal $N_{1}$ neutrinos at $t=t_{R H}, c f$. Eq. (C.2). The background colours reflect the parameter dependence of $\gamma$ as also indicated by the contour lines and labels. In the reddish regions (large $\gamma$ ) the nonthermal neutrinos are relativistic at $t=t_{R H}$, in the bluish regions (small $\gamma$ ) rather nonrelativistic. In principal, $\gamma$ can take on values between 1 and $\frac{1}{2} m_{S} / M_{1} \simeq \frac{1}{2} \eta^{-2} \simeq 150$. Its behavior in parameter space is mainly controlled by $\Gamma_{S}^{0} / \Gamma_{N_{1}}^{0} \propto\left(v_{B-L}^{2} \widetilde{m}_{1}\right) /\left(v_{E W}^{2} M_{1}\right)$. 
The correction factor $\alpha$, the total-to-radiation energy density ratio at $t=t_{R H}$, increases when going to larger $v_{B-L}$ or $\widetilde{m}_{1}$ and decreases for smaller $M_{1}$. It hence essentially shows the same trends in parameter space as $\gamma$. The physical reason for this is that large $\gamma$ implies a rather long-lasting stage of $N_{1}$ production through $S$ decay which persists until shortly before the $N_{1}$ neutrinos decay themselves. Thereby, the $N_{1}$ abundance at $t=t_{R H}$ ends up being still quite large. On top of that, in the case of highly relativistic neutrinos, the effective decay rate $\Gamma_{N_{1}}^{S}$ changes faster with time than for nonrelativistic neutrinos. Or, put into mathematical terms: $\gamma_{t}$ (cf. Eq. (C.2)) is a monotically decreasing function of time which means that large values of $\gamma_{t}$ entail large values of $\frac{d}{d t} \gamma_{t}^{-1}$. Thus, if at $t=t_{R H}$ the decay rate $\Gamma_{N_{1}}^{S}$ equals the Hubble rate $H$ and it is fast changing it was much smaller before. Then not as many nonthermal neutrinos decay at times $t<t_{R H}$ and $\rho_{N_{1}}^{S}\left(t_{R H}\right)$ may contribute much more to $\rho_{\text {tot }}\left(t_{R H}\right)$ than $\rho_{R}\left(t_{R H}\right)$. Numerically, we find that $\alpha$ lies in the range between 3 and 4 for almost two thirds of the investigated parameter space. In approximately $85 \%$ it is of $\mathcal{O}(10)$, in $10 \%$ of $\mathcal{O}(100)$ and in $5 \%$ even larger up to $\alpha \simeq 7.8 \times 10^{4}$. In Eq. C.5 the factor $\alpha^{-1 / 4}$ typically has a size between 0.75 and 0.49. But in extreme cases it can become as small as $\alpha \simeq 6.0 \times 10^{-2}$.

The correction factor in the Friedmann equation $\beta$ turns out to be quite constant in parameter space. We find that it varies between 0.53 and 0.92 . Its standard deviation with respect to its mean value is rather small: $\beta=0.82 \pm 0.06$. A $\beta$ factor smaller than one is the expected consequence of our approach to the calculation of the scale factor: After $t=t_{S}$ we assume pure radiaton domination, $\omega=\rho / p=1 / 3$, although for times $t \gtrsim t_{S}$ surely still some nonrelativistic $S$ bosons contribute to the total energy density. This leads to an overestimation of the speed at which the Hubble rate decreases and in Eq. C.3 to a too small Hubble rate compared to the right-hand side of the equation. A correction factor of $\beta^{-1 / 2} \simeq 1.1$ in Eq. (C.5) is, however, almost insignificant.

In conclusion, we can say that $\gamma$ constitutes the largest correction, followed by $\alpha$, the factor $\beta$ can almost be neglected. This observation leads us to three increasingly accurate approximations for the reheating temperature $T_{R H}^{(0)}, T_{R H}^{(1)}$ and $T_{R H}^{(2)}$, all of which are also shown in Fig. 5. First, we set $\alpha=\beta=\gamma=1$. With $g_{\star, \rho}=915 / 4$ and Eq. (12), we then have

$$
\begin{aligned}
T_{R H}^{(0)} & =\left(\frac{90}{8 \pi^{3} g_{\star, \rho}}\right)^{1 / 4} \sqrt{\Gamma_{N_{1}}^{0} M_{p}} \\
& \simeq 0.2 \sqrt{\Gamma_{N_{1}}^{0} M_{p}} \\
& \simeq 8 \times 10^{9} \mathrm{GeV}\left(\frac{\widetilde{m}_{1}}{10^{-3} \mathrm{eV}}\right)^{1 / 2}\left(\frac{M_{1}}{10^{10} \mathrm{GeV}}\right)
\end{aligned}
$$


This estimate can be improved by including the time dilatation factor $\gamma$,

$$
T_{R H}^{(1)}=\gamma^{-1 / 2} T_{R H}^{(0)} \simeq 0.2 \sqrt{\Gamma_{N_{1}}^{S} M_{p}}
$$

Finally, we drop the assumption that at $t=t_{R H}$ the entire energy resides in radiation,

$$
T_{R H}^{(2)}=\alpha^{-1 / 4} \gamma^{-1 / 2} T_{R H}^{(0)} \simeq \alpha^{-1 / 4} 0.2 \sqrt{\Gamma_{N_{1}}^{S} M_{p}} .
$$

The remaining difference between the outcome of the Boltzmann equations and $T_{R H}^{(2)}$ is then quantified by $\beta$

$$
\begin{aligned}
T_{R H} & =\beta^{-1 / 2} T_{R H}^{(2)} \\
\Rightarrow \quad \log _{10} T_{R H} & =\log _{10} T_{R H}^{(2)}+\Delta \log _{10} T_{R H}, \quad \Delta \log _{10} T_{R H} \simeq 0.04 .
\end{aligned}
$$

To conclude, let us apply the above formulæ to the specific parameter example which we discussed in Section 4. For the parameter values listed in Tab. 2, solving the Boltzmann equations leads to a reheating temperature of $T_{R H}=4.1 \times 10^{9} \mathrm{GeV}$ (cf. Eq. (97)). This result can be compared to the three estimates introduced in this appendix. For the three correction factors $\alpha, \beta$ and $\gamma$ we obtain

$$
\alpha \simeq 3.2, \quad \beta \simeq 0.84, \quad \gamma \simeq 14
$$

At $t=t_{R H}$ there is, hence, still roughly twice as much energy in nonthermal neutrinos as in radiation. Equality in terms of the energy content is not reached before $a \simeq 4000$ in our parameter example. Moreover, with a typical energy $E_{N_{1}} \sim \mathcal{O}(10) M_{1}$ the nonthermal neutrinos are clearly still relativistic during reheating. According to Eq. (C.12) the estimates $T_{R H}^{(0)}, T_{R H}^{(1)}$ and $T_{R H}^{(2)}$ yield reheating temperatures of

$$
T_{R H}^{(0)} \simeq 1.9 \times 10^{10} \mathrm{GeV}, \quad T_{R H}^{(1)} \simeq 5.0 \times 10^{9} \mathrm{GeV}, \quad T_{R H}^{(2)} \simeq 3.7 \times 10^{9} \mathrm{GeV} .
$$

Notice that $T_{R H}^{(2)}$ multiplied by $\beta^{-1 / 2}$ reproduces again the numerical result for the reheating temperature $T_{R H}$ in Eq. (97). We conclude that the most naive estimate $T_{R H}^{(0)}$ is off the actual value by roughly an order of magnitude. $T_{R H}^{(1)}$ and $T_{R H}^{(2)}$ respectively deviate from $T_{R H}$ by $10 \%$ and $20 \%$.

\section{Semi-analytic reconstructions}

Our study of the parameter space in Section 5 allowed us to determine the $N_{1}$ neutrino mass $M_{1}$ and the reheating temperature $T_{R H}$ as functions of $\widetilde{m}_{1}$ and $m_{\widetilde{G}}$ such that the 
gravitino abundance always has the right size to account for dark matter (cf. Figs. 7] and 8). In this appendix we now attempt to reconstruct these results by means of simple analytic expressions and with the aid of our numerical findings for $\eta_{B}$ and $T_{R H}$.

As gravitinos are nonrelativistic, their present contribution to the energy density of the universe is given as

$$
\Omega_{\widetilde{G}} h^{2}=\Omega_{\widetilde{G}} h^{2}\left(v_{B-L}, M_{1}, \widetilde{m}_{1}, m_{\widetilde{G}}, m_{\tilde{g}}\right)=m_{\widetilde{G}} \eta_{\widetilde{G}} n_{\gamma}^{0} h^{2} / \rho_{c}, \quad \eta_{\widetilde{G}}=n_{\widetilde{G}}^{0} / n_{\gamma}^{0} .
$$

In order to relate the gravitino-to-photon ratio $\eta_{\widetilde{G}}$ to the corresponding number densities during reheating we make two simplifying assumptions. First, we say that after $t=t_{R H}$ the entropy of the thermal bath is not increased much further which leads us to

$$
n_{\gamma}^{0}=\delta_{1}\left(\frac{a\left(t_{R H}\right)}{a_{0}}\right)^{3} \frac{g_{\star, s}}{g_{\star, s}^{0}} n_{\gamma}\left(t_{R H}\right) .
$$

Second, we assume that at $t=t_{R H}$ the gravitino production becomes inefficient such that at later times not many further gravitinos are produced,

$$
n_{\widetilde{G}}^{0}=\delta_{2}\left(\frac{a\left(t_{R H}\right)}{a_{0}}\right)^{3} n_{\widetilde{G}}\left(t_{R H}\right) .
$$

Meanwhile, this second assumption also implies that at $t=t_{R H}$ the gravitino production rate $\gamma_{\widetilde{G}}$ is of the same order as the Hubble rate $H$

$$
\frac{\gamma_{\widetilde{G}}\left(t_{R H}\right)}{n_{\widetilde{G}}\left(t_{R H}\right)}=\delta_{3}^{-1} H\left(t_{R H}\right) \Leftrightarrow n_{\widetilde{G}}\left(t_{R H}\right)=\delta_{3} \frac{\gamma_{\widetilde{G}}\left(t_{R H}\right)}{H\left(t_{R H}\right)} .
$$

The three correction factors $\delta_{1} \gtrsim 1, \delta_{2} \gtrsim 1$ and $\delta_{3} \sim \mathcal{O}(1)$, introduced in Eqs. (D.2), (D.3) and (D.4), respectively, quantify the deviations of the actual values of $n_{\gamma}^{0}, n_{\widetilde{G}}^{0}$ and $n_{\widetilde{G}}\left(t_{R H}\right)$ from our approximations. Combining them in one factor $\delta=\delta_{2} \delta_{3} / \delta_{1}$ we may write for $\eta_{\widetilde{G}}$

$$
\eta_{\widetilde{G}}=\delta \frac{g_{\star, s}^{0}}{g_{\star, s}} \frac{\gamma_{\widetilde{G}}\left(t_{R H}\right)}{n_{\gamma}\left(t_{R H}\right) H\left(t_{R H}\right)},
$$

where $n_{\gamma}\left(t_{R H}\right), \gamma_{\widetilde{G}}\left(t_{R H}\right)$ and $H\left(t_{R H}\right)$ directly follow from Eqs. 27), (86) and (C.4). Inserting Eq. (D.5) back into Eq. (D.1) we find for $\Omega_{\widetilde{G}} h^{2}$

$$
\Omega_{\widetilde{G}} h^{2}=\varepsilon f_{\widetilde{G}}\left(T_{R H}\right)\left(m_{\widetilde{G}}+\frac{m_{\tilde{g}}^{2}\left(T_{R H}\right)}{3 m_{\widetilde{G}}}\right) T_{R H}, \quad \varepsilon=\alpha^{-1 / 2} \beta^{-1} \delta,
$$

where $f_{\widetilde{G}}\left(T_{R H}\right)$ stands for

$$
f_{\widetilde{G}}\left(T_{R H}\right)=\frac{n_{\gamma}^{0} h^{2}}{\rho_{c}} \frac{g_{\star, s}^{0}}{g_{\star, s}}\left(\frac{90}{8 \pi^{3} g_{\star, \rho}}\right)^{1 / 2} \frac{54 g_{s}^{2}\left(T_{R H}\right)}{g_{\gamma} M_{p}}\left[\ln \left(\frac{T_{R H}^{2}}{m_{g}^{2}\left(T_{R H}\right)}\right)+0.8846\right] .
$$


Eq. (D.6) may conveniently be rewritten as

$$
\Omega_{\widetilde{G}} h^{2}=\varepsilon C_{1}\left(T_{R H}\right)\left(\frac{T_{R H}}{10^{9} \mathrm{GeV}}\right)\left[C_{2}\left(T_{R H}\right)\left(\frac{m_{\widetilde{G}}}{10 \mathrm{GeV}}\right)+\left(\frac{10 \mathrm{GeV}}{m_{\widetilde{G}}}\right)\left(\frac{m_{\tilde{g}}\left(\mu_{0}\right)}{800 \mathrm{GeV}}\right)^{2}\right]
$$

with $C_{1}$ and $C_{2}$ being defined as

$$
\begin{aligned}
& C_{1}\left(T_{R H}\right)=\frac{(800 \mathrm{GeV})^{2}}{10 \mathrm{GeV}} \times 10^{9} \mathrm{GeV} \times \frac{g_{s}^{4}\left(T_{R H}\right)}{3 g_{s}^{4}\left(\mu_{0}\right)} \times f_{\tilde{G}}\left(T_{R H}\right), \\
& C_{2}\left(T_{R H}\right)=\frac{10 \mathrm{GeV}}{(800 \mathrm{GeV})^{2}} \times 10 \mathrm{GeV} \times \frac{3 g_{s}^{4}\left(\mu_{0}\right)}{g_{s}^{4}\left(T_{R H}\right)} .
\end{aligned}
$$

The dependence of $C_{1}$ and $C_{2}$ on the reheating temperature is presented in Fig. 11 . We find that $C_{1} / C_{2} \sim \mathcal{O}(100)$ which means that for $m_{\tilde{g}} \gg m_{\widetilde{G}}$ the term linear in $m_{\widetilde{G}}$ in Eq. (D.8) can usually be neglected. Notice that doing so and setting $\varepsilon=1$ turns Eq. (D.8) into Eq. (3) in the introduction.

Confronting Eq. (D.8) with our numerical data shows that $\varepsilon$ usually differs from 1 and is slightly parameter-dependent, preventing us from determining it a priori. However, $\varepsilon$ can be determined a posteriori. In the region of parameter space in which $\Omega_{\widetilde{G}} h^{2}=\Omega_{D M} h^{2}$ we find that $\varepsilon=1.20 \pm 0.24$. Restricting ourselves further to parameter values for which gravitino dark matter also is in accordance with successful leptogenesis we obtain $\varepsilon=$ $1.32 \pm 0.15$. In Eq. (3) such a correction factor would be reflected in a change of the numerical coefficient from 0.26 to 0.34 .

Eq. (D.8) then implicitly determines the reheating temperature as a function of $m_{\widetilde{G}}$ and $m_{\tilde{g}}\left(\mu_{0}\right)$. Fixing the gluino mass at $800 \mathrm{GeV}$ and solving Eq. (D.8) for $T_{R H}$ provides us with an estimate for the reheating temperature solely dependent on $m_{\widetilde{G}}$ (cf. Fig. 12 ). This is all we need to be able to reconstruct Figs. 5 and 6 ; With $T_{R H}=T_{R H}\left(m_{\widetilde{G}}\right)$ at hand we can compute the reheating temperature for all values of the parameter triple $\left(v_{B-L}, \widetilde{m}_{1}, m_{\widetilde{G}}\right)$. From our numerical results for $T_{R H}$ as a function of $v_{B-L}, M_{1}$ and $\widetilde{m}_{1}$, shown in Fig. 5, we then infer the corresponding values of $M_{1}$. Our results for $\eta_{B}$ in Fig. 6 finally point us to the respective baryon asymmetries

$$
\begin{aligned}
\Omega_{\widetilde{G}} h^{2} \stackrel{!}{=} 0.11 & \Rightarrow \quad T_{R H}=T_{R H}\left(m_{\widetilde{G}}\right), \\
& \Rightarrow \quad M_{1}=M_{1}\left(v_{B-L}, T_{R H}, \widetilde{m}_{1}\right) \\
& \Rightarrow \quad \eta_{B}=\eta_{B}\left(v_{B-L}, M_{1}, \widetilde{m}_{1}\right)
\end{aligned}
$$

The outcome of this procedure is presented in Figs. 13 and 14 . As it turns out our reconstructed results fit the exact data from the Boltzmann equations amazingly well. We thus conclude that our numerical results for the reheating temperature $T_{R H}$ and the 
Coefficients in the $\Omega_{\tilde{G}} h^{2}$ estimate

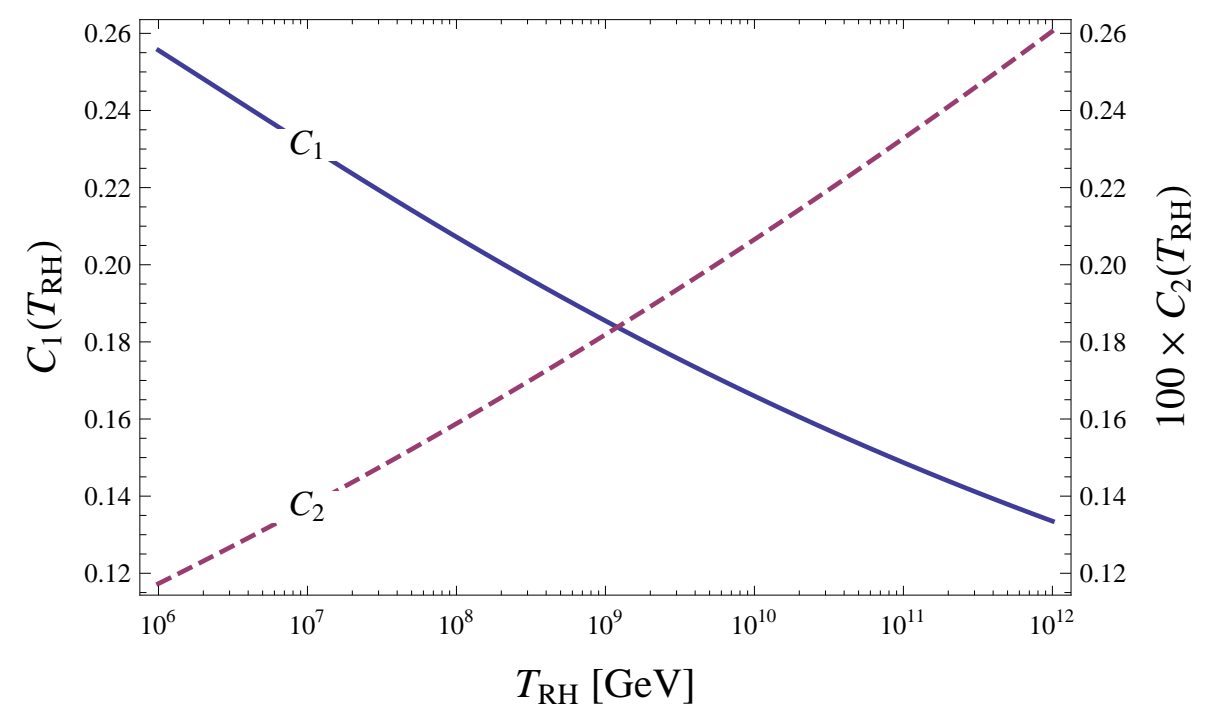

Figure 11: Dependence of the coefficients $C_{1}$ and $C_{2}$ in Eq. (D.8) on the reheating temperature $T_{R H}$.

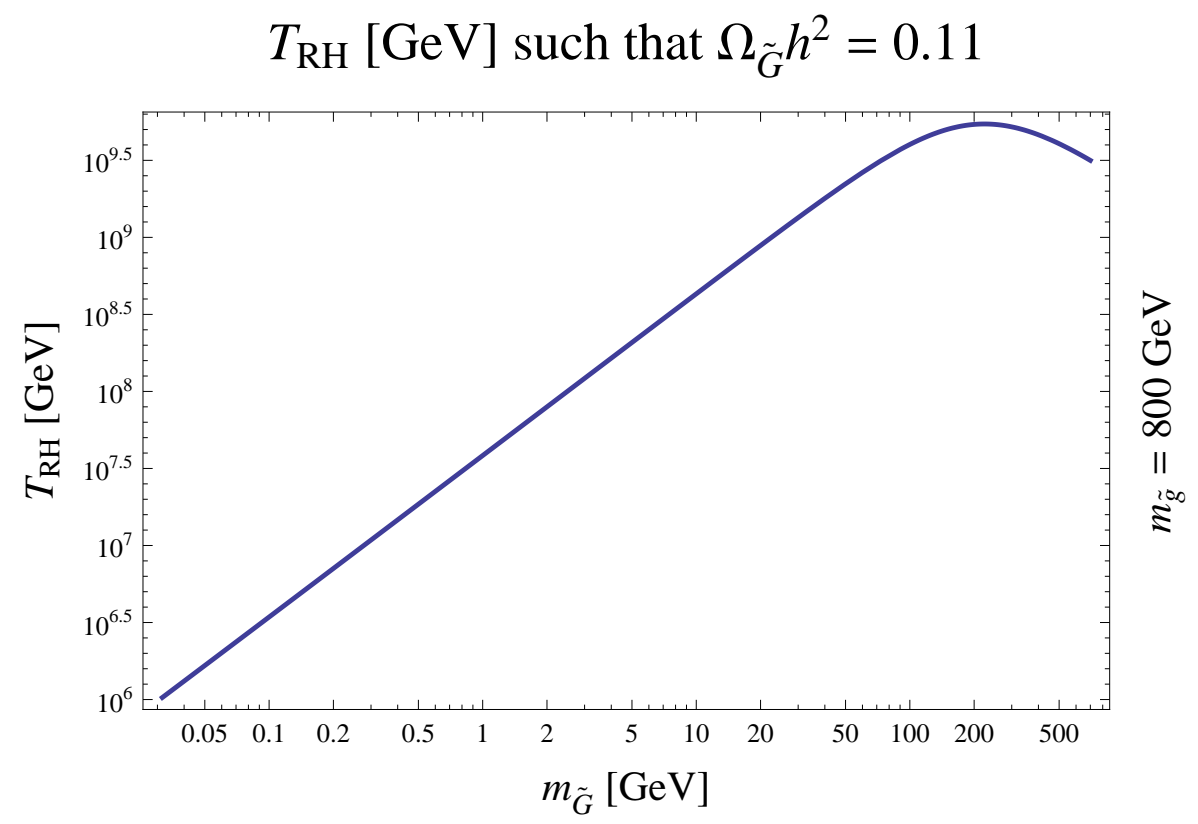

Figure 12: Analytic estimate for the reheating temperature $T_{R H}$ as implicitly defined by Eq. (D.8) with $\varepsilon=1.32$ for varying gravitino mass $m_{\widetilde{G}}$ and fixed gluino mass, $m_{\tilde{g}}=800 \mathrm{GeV}$. 
baryon asymmetry $\eta_{B}$ when combined with Eq. (D.8) essentially suffice to reproduce Figs. 7 and 8 .

Let us check how well the result for $\Omega_{\widetilde{G}} h^{2}$ (cf. Eq. (D.8)) that we obtained for the parameter example discussed in Section 4 can be reproduced with the formulæ developed in this appendix. The three correction factors $\delta_{1}, \delta_{2}$ and $\delta_{3}$ turn out to be

$$
\delta_{1}=\frac{N_{\gamma}\left(t_{f}\right)}{N_{\gamma}\left(t_{R H}\right)} \simeq 2.7, \quad \delta_{2}=\frac{N_{\widetilde{G}}\left(t_{f}\right)}{N_{\widetilde{G}}\left(t_{R H}\right)} \simeq 15, \quad \delta_{3}=\frac{H\left(t_{R H}\right) n_{\widetilde{G}}\left(t_{R H}\right)}{\gamma_{\widetilde{G}}\left(t_{R H}\right)} \simeq 0.35,
$$

which implies that entropy production has almost completed at $t=t_{R H}$. By contrast the gravitino production rate is still roughly three times as large as the Hubble rate at this time such that the bulk part of the gravitinos is, in fact, produced at the last stages of reheating and later. In combination with Eq. (C.12) the three factors yield values of $\delta$ and $\varepsilon$ of

$$
\delta=\delta_{2} \delta_{3} / \delta_{1} \simeq 2.0, \quad \varepsilon=\alpha^{-1 / 2} \beta^{-1} \delta \simeq 1.3
$$

This result for $\varepsilon$ coincides with the fit value used for the reconstruction of Figs. 13 and 14. Notice also that the effects of the various approximations parametrized by $\alpha$ and $\delta$ tend to cancel such that overall factor $\varepsilon$ represents a correction of only $30 \%$ in the end. Given the reheating temperature in Eq. (97) the coefficients $C_{1}$ and $C_{2}$ in Eq. (D.8) take on the following values

$$
C_{1}\left(T_{R H}\right) \simeq 0.17, \quad C_{2}\left(T_{R H}\right) \simeq 2.0 \times 10^{-3} .
$$

Based on Eq. (D.8) we can then estimate the gravitino abundance

$$
\begin{aligned}
\Omega_{\widetilde{G}} h^{2} & =1.3 \times 0.17\left(\frac{T_{R H}}{10^{9} \mathrm{GeV}}\right)\left[0.002\left(\frac{m_{\widetilde{G}}}{10 \mathrm{GeV}}\right)+\left(\frac{10 \mathrm{GeV}}{m_{\widetilde{G}}}\right)\left(\frac{m_{\tilde{g}}\left(\mu_{0}\right)}{800 \mathrm{GeV}}\right)^{2}\right] \\
& \simeq 1.3 \times 0.17 \times 4.1 \times(0.02+0.1) \simeq 0.11
\end{aligned}
$$

which is exactly the value we obtained solving the Boltzmann equations. Without the correction factor $\varepsilon$, we would end up with a too small value, $\Omega_{\widetilde{G}} h^{2} \simeq 8.5 \times 10^{-2}$. If we were to neglect the term linear in $m_{\widetilde{G}}$ in addition, our estimate would come out even smaller, $\Omega_{\widetilde{G}} h^{2} \simeq 7.1 \times 10^{-2}$. In concluding, we also mention that our result for the reheating temperature in Eq. (97) coincides, by construction, with the value required for gravitino dark matter: In Fig. 12 we read off that to $m_{\widetilde{G}}=100 \mathrm{GeV}$ corresponds a temperature of $T_{R H} \simeq 4.0 \times 10^{9} \mathrm{GeV}$. 

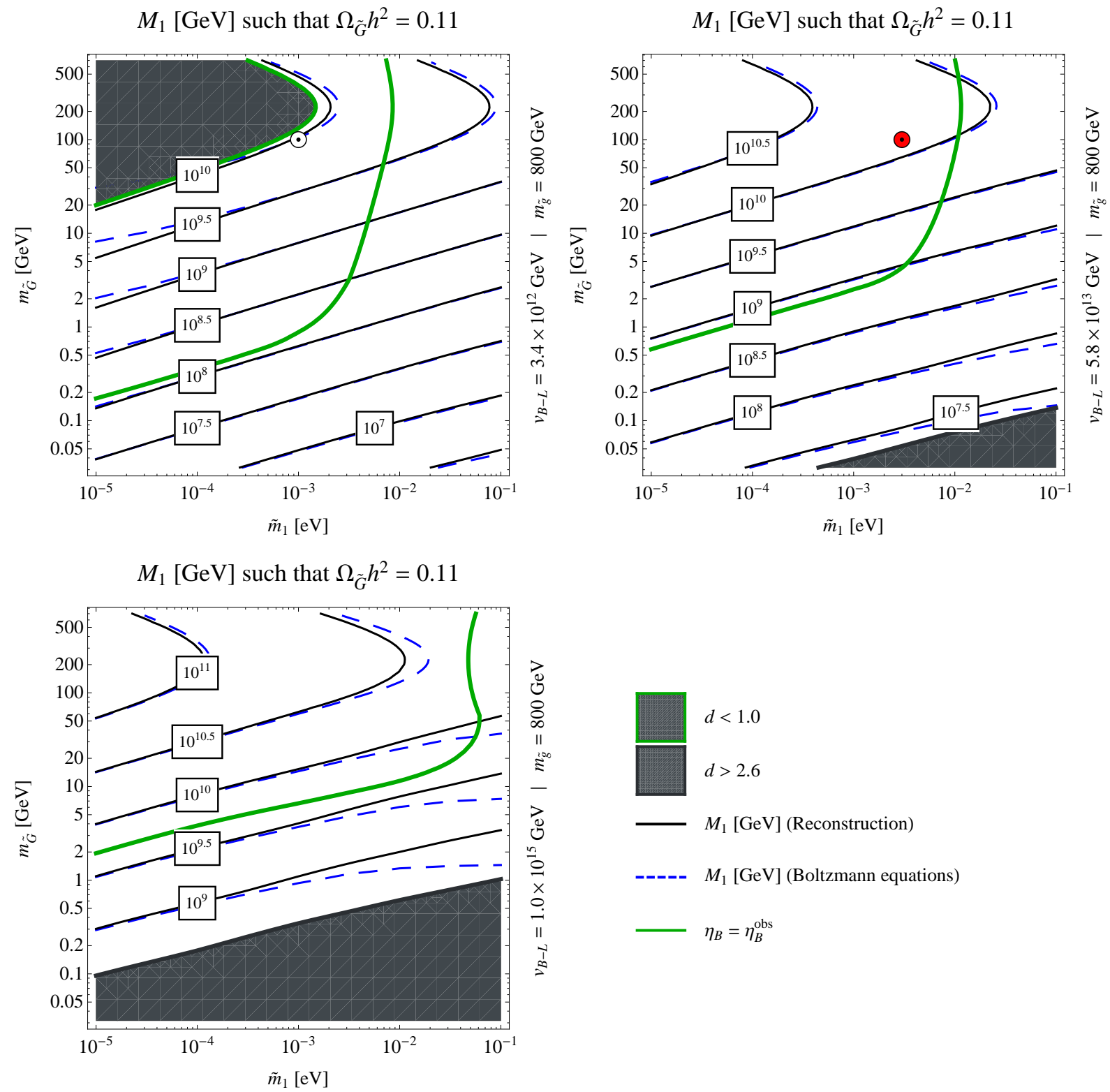

$\longrightarrow \quad M_{1}[\mathrm{GeV}]$ (Reconstruction)

----- $M_{1}[\mathrm{GeV}]$ (Boltzmann equations)

$\eta_{B}=\eta_{B}^{\mathrm{obs}}$

Figure 13: Semi-analytical reconstruction of Fig. 7 (solid lines) on the basis of Eq. D.8) with $\varepsilon=1.32$ and the numerical results for $T_{R H}$ and $\eta_{B}$. For comparison also the $M_{1}$ contours deduced from the Boltzmann equations (dashed lines) are shown. They deviate from the reconstructed results as the correction factor $\varepsilon=\alpha^{-1 / 2} \beta^{-1} \delta$ actually is parameter-dependent. 

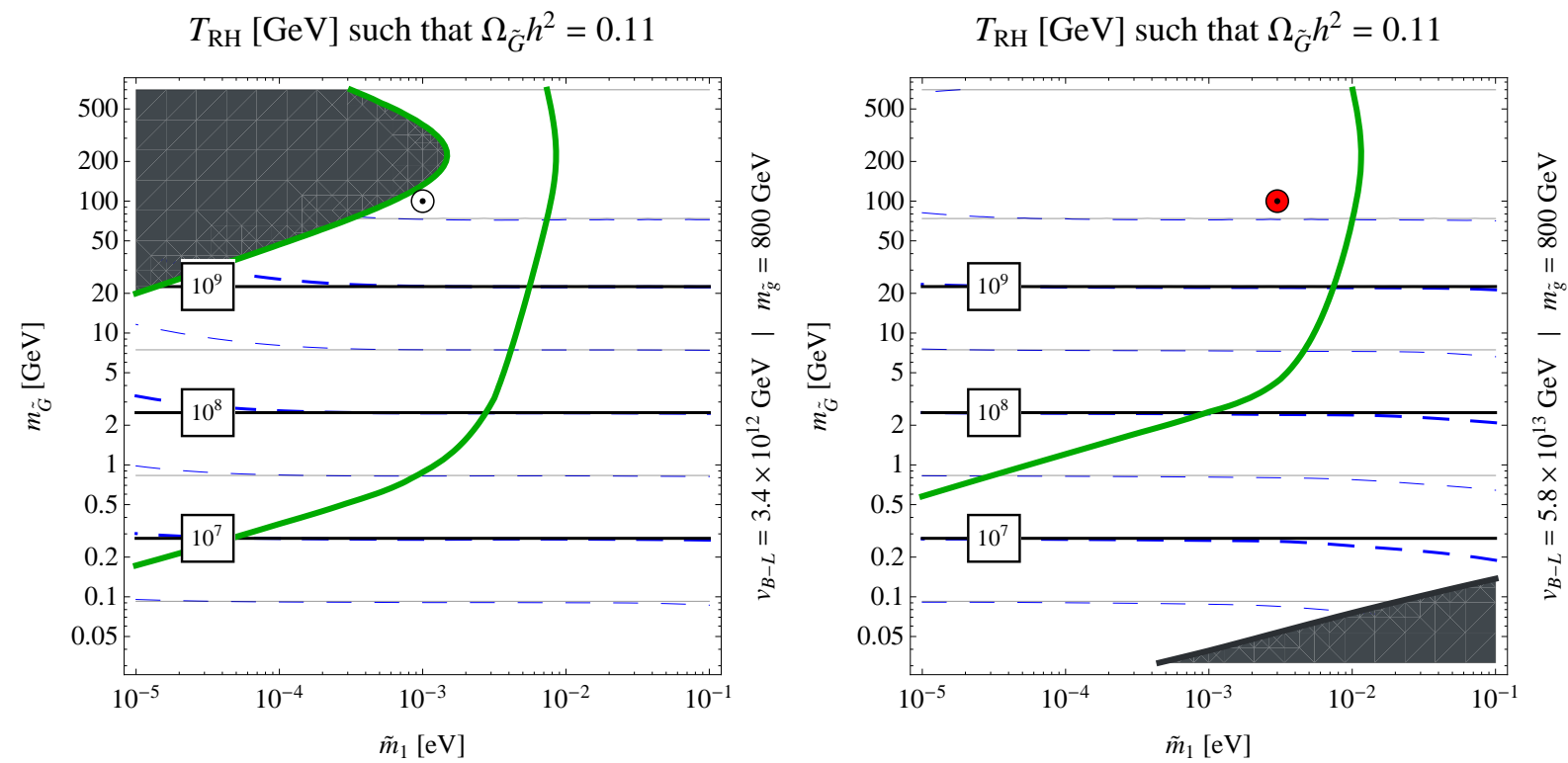

$T_{\mathrm{RH}}[\mathrm{GeV}]$ such that $\Omega_{\tilde{G}} h^{2}=0.11$

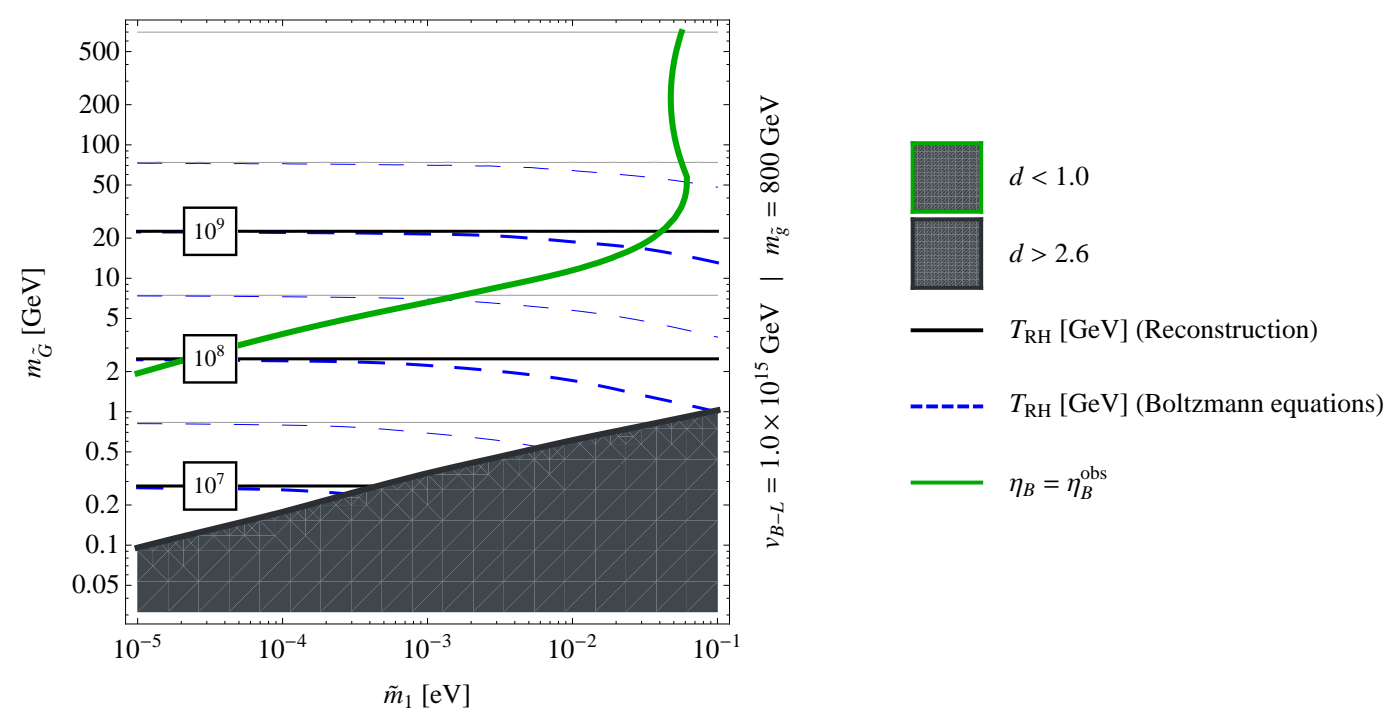

Figure 14: Semi-analytical reconstruction of Fig. 8 (solid lines) on the basis of Eq. (D.8) with $\varepsilon=1.32$ and the numerical results for $T_{R H}$ and $\eta_{B}$. For comparison also the $T_{R H}$ contours deduced from the Boltzmann equations (dashed lines) are shown. They deviate from the reconstructed results as the correction factor $\varepsilon=\alpha^{-1 / 2} \beta^{-1} \delta$ actually is parameter-dependent. 
Finally, our results may be easily generalized to gluino masses other than $800 \mathrm{GeV}$. In fact, for given values of $v_{B-L}, \widetilde{m}_{1}, M_{1}$ and $m_{\widetilde{G}}$ it is possible to keep $\eta_{B}$ and $\Omega_{\widetilde{G}} h^{2}$ constant, when changing $m_{\tilde{g}}$, by simply rescaling the gravitino mass,

$$
m_{\widetilde{G}}^{0} \rightarrow m_{\widetilde{G}}=m_{\widetilde{G}}\left(m_{\tilde{g}}, m_{\widetilde{G}}^{0}\right), \quad m_{\widetilde{G}}\left(800 \mathrm{GeV}, m_{\widetilde{G}}^{0}\right)=m_{\widetilde{G}}^{0} .
$$

As for the baryon asymmetry, this is a trivial consequence of the fact that $\eta_{B}$ is a function of $v_{B-L}, \widetilde{m}_{1}$ and $M_{1}$ only. In the case of the gravitino abundance we observe that for fixed reheating temperature, $T_{R H}=T_{R H}\left(v_{B-L}, \widetilde{m}_{1}, M_{1}\right), \Omega_{\widetilde{G}} h^{2}$ remains constant as long as $m_{\widetilde{G}}\left(m_{\tilde{g}}, m_{\widetilde{G}}^{0}\right)$ is chosen such that the term in square brackets in Eq. (D.8) does not change,

$$
\left[C_{2}\left(\frac{m_{\widetilde{G}}^{0}}{10 \mathrm{GeV}}\right)+\left(\frac{10 \mathrm{GeV}}{m_{\widetilde{G}}^{0}}\right)\right]=\left[C_{2}\left(\frac{m_{\widetilde{G}}}{10 \mathrm{GeV}}\right)+\left(\frac{10 \mathrm{GeV}}{m_{\widetilde{G}}}\right)\left(\frac{m_{\tilde{g}}}{800 \mathrm{GeV}}\right)^{2}\right],
$$

where $C_{2}=C_{2}\left(T_{R H}\right)$ is defined in Eq. (D.10). From Eq. (D.16) we can determine the rescaled gravitino mass $m_{\widetilde{G}}$ as a function of the rescaled gluino mass $m_{\tilde{g}}$ and the original gravitino mass $m_{\widetilde{G}}^{0}$. As Eq. (D.16) is a quadratic equation in $m_{\widetilde{G}}$, it generically has two solutions $m_{\widetilde{G}}^{ \pm}$, one of which is typically closer to the original gravitino mass than the other. $m_{\widetilde{G}}^{0}$ lies right in between $m_{\widetilde{G}}^{-}$and $m_{\widetilde{G}}^{+}$once the two terms in square brackets in Eq. (D.8) are of equal size, i.e. when gravitinos in helicity $\pm \frac{1}{2}$ states contribute exactly as much to the total abundance as gravitinos in helicity $\pm \frac{3}{2}$ states. One easily sees that this is the case when $m_{\widetilde{G}}^{0} \simeq 230 \mathrm{GeV}$ (cf. Eq. (117)). When going to larger $m_{\tilde{g}}$, we have $m_{\widetilde{G}}^{0} \gtrsim m_{\widetilde{G}}^{+} \gg m_{\widetilde{G}}^{-}$above $230 \mathrm{GeV}$ and $m_{\widetilde{G}}^{0} \lesssim m_{\widetilde{G}}^{-} \ll m_{\widetilde{G}}^{+}$below $230 \mathrm{GeV}$. At $m_{\tilde{g}}$ smaller than $800 \mathrm{GeV}$, we always find $m_{\widetilde{G}}^{-}<m_{\widetilde{G}}^{0}<m_{\widetilde{G}}^{+}$.

If the gravitino mass is much smaller than the gluino mass, almost only the goldstino part of the gravitino is produced and the term linear in $m_{\widetilde{G}}$ in Eq. (D.8) can be neglected. The scaling behaviour of the gravitino mass then becomes trivial

$$
m_{\widetilde{G}}^{0} \ll m_{\tilde{g}}: \quad m_{\widetilde{G}}=m_{\widetilde{G}}^{0}\left(\frac{m_{\tilde{g}}}{800 \mathrm{GeV}}\right)^{2} .
$$

Actually, the rescaled gravitino mass $m_{\widetilde{G}}$ also is a function of $T_{R H}$ as it depends on the coefficient $C_{2}\left(T_{R H}\right)$. But as discussed in this appendix, there is an almost unique correspondence between the gravitino mass and the reheating temperature. In order to solve Eq. (D.16) we thus simply read off $T_{R H}$ from Fig. 12 as a function of the input gravitino mass, $T_{R H}=T_{R H}\left(m_{\widetilde{G}}^{0}\right)$. As can be seen from Fig. 14 , this simplified reheating temperature is in good agreement with the exact outcome of the Boltzmann equations. Our solutions $m_{\widetilde{G}}^{ \pm}$for the rescaled gravitino mass are presented in the two panels of Fig. 15 . 

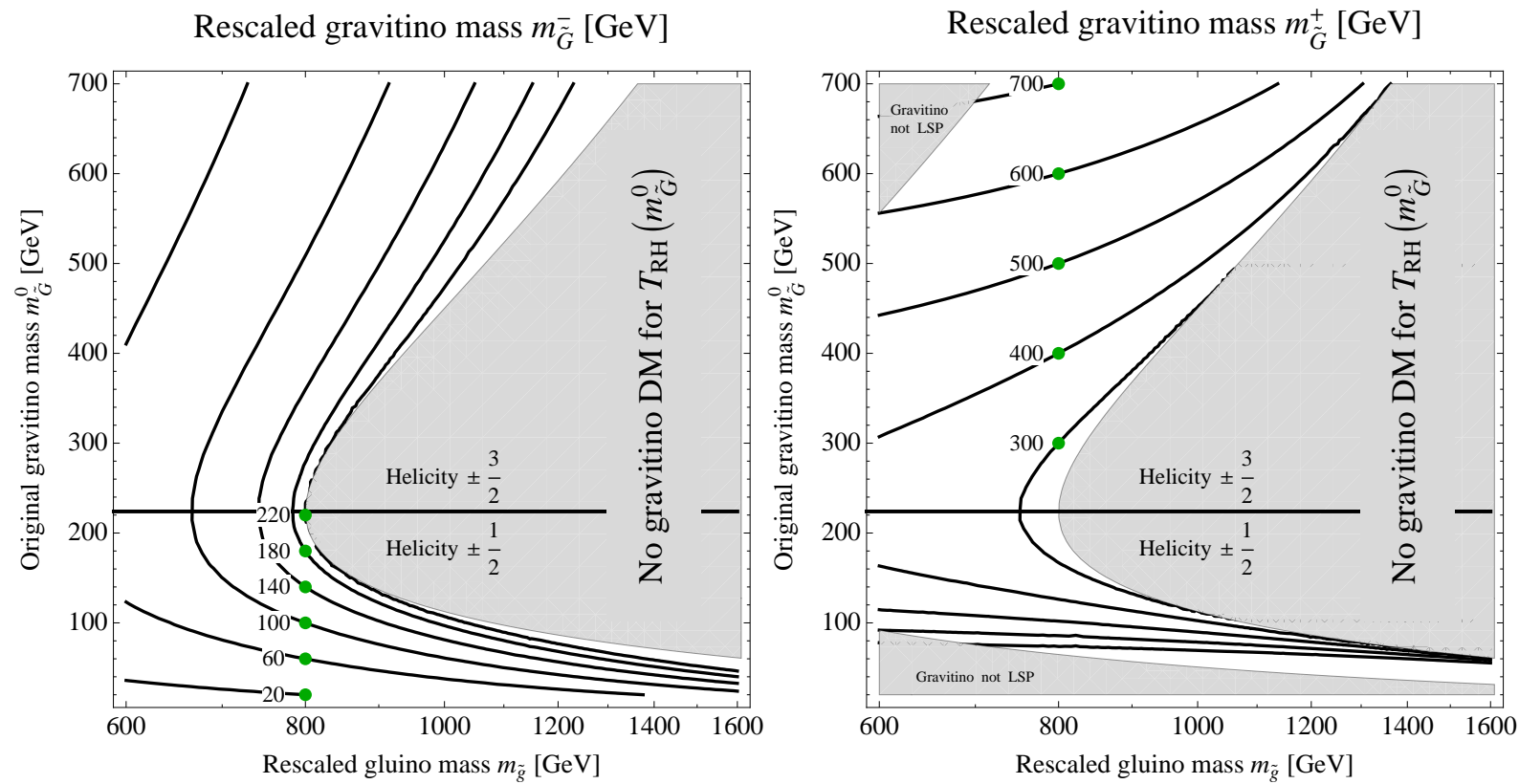

Figure 15: Contour plots of the two solutions $\left(m_{\widetilde{G}}^{ \pm}\right)$of Eq. D.16) for the rescaled gravitino mass $m_{\widetilde{G}}$ as a function of the rescaled gluino mass $m_{\tilde{g}}$ and the original gravitino mass $m_{\widetilde{G}}^{0}$. The black solid contours correspond to constant values of $m_{\widetilde{G}}$ (given next to the green dots). They serve as level curves that allow a determination of $m_{\widetilde{G}}$ for arbitrary points in the $\left(m_{\tilde{g}}, m_{\widetilde{G}}^{0}\right)$-plane. They can also be regarded as function graphs of $m_{\widetilde{G}}^{0}$ as a function of $m_{\tilde{g}}$ for constant $m_{\widetilde{G}}$. We restrict ourselves to the interval $20 \mathrm{GeV} \leq m_{\widetilde{G}}^{0} \leq 700 \mathrm{GeV}$ in this figure. Below $20 \mathrm{GeV}$, Eq. D.17 provides an excellent approximation.

respectively. In the gray shaded regions there are either no real solutions of Eq. (D.16) or the rescaled gravitino mass is larger than the corresponding gluino mass, $m_{\widetilde{G}}>m_{\tilde{g}}$. The former case implies that it is impossible to keep the gravitino abundance constant when going to larger $m_{\tilde{g}}$ while sticking to the reheating temperature $T_{R H}\left(m_{\widetilde{G}}^{0}\right)$. In the latter case, the gravitino would not be the LSP any longer. 


\section{References}

[1] Review of particle physics, J. Phys. G 37 (2010) 075021.

[2] For a review and references see, for example,

A. D. Linde, Lect. Notes Phys. 738 (2008) 1; L. Kofman, ibid. p.55.

[3] W. Buchmuller, K. Schmitz and G. Vertongen, Phys. Lett. B 693 (2010) 421 [1008.2355 [hep-ph]].

[4] M. Fukugita and T. Yanagida, Phys. Lett. B 174, 45 (1986).

[5] For a review and references see, for example,

W. Buchmuller, R. D. Peccei and T. Yanagida, Ann. Rev. Nucl. Part. Sci. 55 (2005) 311 [hep-ph/0502169];

S. Davidson, E. Nardi and Y. Nir, Phys. Rept. 466 (2008) 105 [0802.2962 [hep-ph]].

[6] G. Lazarides and Q. Shafi, Phys. Lett. B 258 (1991) 305.

[7] T. Asaka, K. Hamaguchi, M. Kawasaki and T. Yanagida, Phys. Lett. B 464 (1999) 12 [hep-ph/9906366]; Phys. Rev. D 61 (2000) 083512 [hep-ph/9907559];

F. Hahn-Woernle and M. Plumacher, Nucl. Phys. B 806 (2009) 68 [0801.3972 [hep$\mathrm{ph}]$.

[8] H. Murayama, H. Suzuki, T. Yanagida and J. Yokoyama, Phys. Rev. Lett. 70 (1993) 1912;

J. R. Ellis, M. Raidal and T. Yanagida, Phys. Lett. B 581 (2004) 9 [hep-ph/0303242].

[9] S. Antusch, M. Bastero-Gil, S. F. King and Q. Shafi, Phys. Rev. D 71 (2005) 083519 [hep-ph/0411298];

S. Antusch, J. P. Baumann, V. F. Domcke and P. M. Kostka, JCAP 1010 (2010) 006 [1007.0708 [hep-ph]].

[10] K. Hamaguchi, H. Murayama and T. Yanagida, Phys. Rev. D 65 (2002) 043512 [hep-ph/0109030].

[11] S. Weinberg, Phys. Rev. Lett. 48 (1982) 1303.

[12] J. R. Ellis, D. V. Nanopoulos and S. Sarkar, Nucl. Phys. B 259 (1985) 175. 
[13] M. Kawasaki, K. Kohri and T. Moroi, Phys. Lett. B 625, 7 (2005) [astro-ph/0402490]; Phys. Rev. D 71, 083502 (2005) [astro-ph/0408426];

K. Jedamzik, Phys. Rev. D 74, 103509 (2006) [hep-ph/0604251].

[14] M. Bolz, W. Buchmuller and M. Plumacher, Phys. Lett. B 443 (1998) 209 [hep$\mathrm{ph} / 9809381]$.

[15] M. Bolz, A. Brandenburg and W. Buchmuller, Nucl. Phys. B 606, 518 (2001) [Erratum-ibid. B 790, 336 (2008)] [hep-ph/0012052].

[16] J. Pradler and F. D. Steffen, Phys. Rev. D 75, 023509 (2007) [hep-ph/0608344].

[17] V. S. Rychkov and A. Strumia, Phys. Rev. D 75, 075011 (2007) [hep-ph/0701104].

[18] J. L. Feng, S. Su and F. Takayama, Phys. Rev. D 70 (2004) 075019 [hep-ph/0404231].

[19] F. D. Steffen, Eur. Phys. J. C 59 (2009) 557 [0811.3347 [hep-ph]].

[20] E. Komatsu et al. [WMAP Collaboration], Astrophys. J. Suppl. 192 (2011) 18 [1001.4538 [astro-ph.CO]].

[21] G. N. Felder, J. Garcia-Bellido, P. B. Greene, L. Kofman, A. D. Linde and I. Tkachev, Phys. Rev. Lett. 87 (2001) 011601 [hep-ph/0012142]; G. N. Felder, L. Kofman and A. D. Linde, Phys. Rev. D 64 (2001) 123517 [hep-th/0106179].

[22] W. Buchmüller and T. Yanagida, Phys. Lett. B 445 (1999) 399 [hep-ph/9810308].

[23] W. Buchmuller, D. Delepine and L. T. Handoko, Nucl. Phys. B 576 (2000) 445 [hep-ph/9912317].

[24] L. Covi, E. Roulet and F. Vissani, Phys. Lett. B 384 (1996) 169 [hep-ph/9605319].

[25] W. Buchmuller and M. Plumacher, Phys. Lett. B 431 (1998) 354 [hep-ph/9710460].

[26] S. Davidson and A. Ibarra, Phys. Lett. B 535 (2002) 25 [hep-ph/0202239].

[27] M. Fujii, K. Hamaguchi and T. Yanagida, Phys. Rev. D 65 (2002) 115012 [hep$\mathrm{ph} / 0202210]$.

[28] J. Garcia-Bellido and E. Ruiz Morales, Phys. Lett. B 536 (2002) 193 [hep$\mathrm{ph} / 0109230]$.

[29] M. Plumacher, Nucl. Phys. B 530 (1998) 207 [arXiv:hep-ph/9704231]. 
[30] W. Buchmuller, V. Domcke, K. Schmitz and G. Vertongen, Work in preparation.

[31] For a discussion and references see, for example, R. Jeannerot, hep-ph/0610295.

[32] S. Ferrara, R. Kallosh, A. Linde, A. Marrani and A. Van Proeyen, Phys. Rev. D 82 (2010) 045003 [arXiv:1004.0712 [hep-th]].

[33] W. Buchmuller, P. Di Bari and M. Plumacher, Annals Phys. 315 (2005) 305 [hep$\mathrm{ph} / 0401240]$.

[34] F. Hahn-Woernle, M. Plumacher and Y. Y. Y. Wong, JCAP 0908 (2009) 028 [0907.0205 [hep-ph]].

[35] V. Khachatryan et al. [CMS Collaboration], Phys. Lett. B 698 (2011) 196 [1101.1628 [hep-ex]].

[36] G. Aad et al. [Atlas Collaboration], 1102.2357 [hep-ex].

[37] E. W. Kolb and M. S. Turner, The Early universe, Front. Phys. 69 (1990) 1.

[38] K. Nakayama, F. Takahashi and T. T. Yanagida, JCAP 1012 (2010) 010 [1007.5152 [hep-ph]].

[39] L. Covi, J. Hasenkamp, S. Pokorski and J. Roberts, JHEP 0911 (2009) 003 [0908.3399 [hep-ph]].

[40] M. Pospelov, Phys. Rev. Lett. 98 (2007) 231301 [hep-ph/0605215].

[41] R. H. Cyburt, J. R. Ellis, B. D. Fields, K. A. Olive and V. C. Spanos, JCAP 0611 (2006) 014 [astro-ph/0608562].

[42] A. Anisimov, W. Buchmuller, M. Drewes and S. Mendizabal, 1012.5821 [hep-ph]. 